UNIVERSITE DU QUEBBEC

\author{
MEMOIRE \\ PRÉSENTE A \\ L'UNIVERSITE DU QUEBEC A CHICOUTIMI \\ COMME EXIGENCE PARTIELLE \\ DE LA MAITRISE EN GESTION DES PMO
}

\author{
PAR \\ BERNARD FLAMAND \\ B. en Pédagogie \\ B. Sp en administration
}

VALEUR ET IMPORTANCE DU POINT DE SERVICES

DE LA CAISSE POPULAIRE D'ARVIDA

A SAINT-JEAN-EUDES

AVRIL 1987 


\section{Bibliothèque}

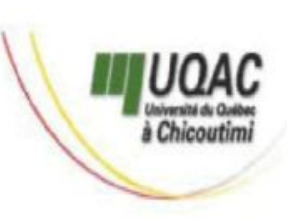

\section{Mise en garde/Advice}

Afin de rendre accessible au plus Motivated by a desire to make the grand nombre le résultat des results of its graduate students' travaux de recherche menés par ses research accessible to all, and in étudiants gradués et dans l'esprit des accordance with the rules règles qui régissent le dépôt et la governing the acceptation and diffusion des mémoires et thèses diffusion of dissertations and produits dans cette Institution, theses in this Institution, the I'Université du Québec à Université du Québec à Chicoutimi (UQAC) est fière de Chicoutimi (UQAC) is proud to rendre accessible une version make a complete version of this complète et gratuite de cette œuvre. work available at no cost to the reader.

L'auteur conserve néanmoins la The author retains ownership of the propriété du droit d'auteur qui copyright of this dissertation or protège ce mémoire ou cette thèse. thesis. Neither the dissertation or Ni le mémoire ou la thèse ni des thesis, nor substantial extracts from extraits substantiels de ceux-ci ne it, may be printed or otherwise peuvent être imprimés ou autrement reproduced without the author's reproduits sans son autorisation. permission. 
RESSUME

Depuis la fondation de leur caisse populaire en 1950, jusqu'à la fusion avec la Caisse populaire d'Arvida en 1973, les citoyens de Saint-Jean-Eudes s'étaient dotés de services financiers parmi les plus complets au niveau de l'épargne et du crédit. En 1980, 1a caisse populaire d'Arvida abandonnait à son Point de services de Saint-JeanEudes les services spécialisés d'épargne et de crédit, n'y laissant que les services courants de dépôts et de retraits.

Une analyse des principales composantes du Point de services aura permis de dégager certaines constantes et attentes face au fonctionnement du dit Point de services.

A I'aide des renseignements obtenus, nous avons pu dégager une problématique révélant certaines faiblesses techniques comme les files d'attente, le stationnement, la communication avec les clients ou encore la circulation de I'air. Les renseignements financiers nous ont donné l'assurance de la valeur du Point de services. D'autres renseignements nous ont assuré de son importance pour les gens du secteur.

Ainsi donc, toutes les informations recueillies ont amené la proposition d'un modèle conceptuel de services financiers répondant à la situation et aux besoins des gens du milieu.

La finalité de notre démarche étant de maintenir, voire accroitre la valeur du Point de services, nous proposons la mise en place d'un système de services financiers incluant les facilités d'emprunter et 
la possibilité de faire des placements à termes. Afin de rendre cet objectif réalisable nous suggérons à la Caisse populaire d'Arvida de procéder à des rénovations de l'intérieur de ses locaux à Saint-Jean-Eudes pour permettre l'entrée en fonction d'un agent d'épargne et de crédit auquel on aura confié la responsabilité du développement futur du Point de services.

Les engagements financiers se situeraient aux environ de 25000 \$ la première année. 


\section{DEDICACE}

Je dédie ce mémoire à mon épouse Lise ainsi qu'à mes cinq enfants: Nathalie Rémy, Nicolas, Matthieu et Vanina. Pendant quatre ans, ils ont accepté d'être privés d'une présence aimante régulière. Sans leur acceptation, il aurait été impossible de produire cette recherche-action.

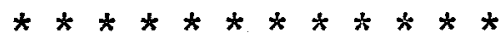

"Toute action bénévole est un pari sur l'humain, un geste de foi en la beauté et la dignité de la personne humaine."

Raymond Blais

président du mouvement Desjardins 


\section{REMERCIEMENTS}

La rédaction d'une thèse de maîtrise ne peut se faire sans la collaboration de nombreuses personnes. Le support technique et moral dont j'ai été entouré au cours des quatre années qu'a duré la production de cette recherche constitue pour moi un acte de foi dans la dignité humaine. C'est ce qui permet à l'humain de contribuer à l'édification d'un univers meilleur.

Je remercie donc toutes les personnes qui de près ou de loin ont contribué à la rédaction de ce mémoire, Mais je ne saurais passer sous silence certaines d'entre elles.

Je dis d'abord un grand merci à mon épouse Lise et mes enfants. Leur patience, leur encouragement et leur abnégation constante, ont été pour moi des modèles de persévérance.

Des remerciements spéciaux s'adressent à mon directeur de thèse, Monsieur Gilles Comtois. Ses judicieux conseils ont ajouté beaucoup à la qualité du travail présenté.

Je remercie également le directeur de la Caisse populaire d'Arvida, Monsieur Marc Laflamme. Sa disponibilité m'a permis de fournir des renseignements plus précis aux lecteurs.

Je remercie enfin Madame Carole Côté pour son apport constant dans la recherche des données statistiques. 
RESUME

Page

DEDICACE . . . . . . . . . . . . . . . . . . . . .

REMERCIEMENTS . . . . . . . . . . . . . . . . . . . iv

TABLE DES MATIERES . . . . . . . . . . . . . . . . . . . V v

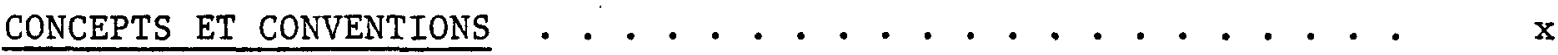

LISTE DES TABLEAUX . . . . . . . . . . . . . . . . . xii

LISTE DE FIGURES . . . . . . . . . . . . . . . . . . xiii

INTRODUCTION . . . . . . . . . . . . . . . . . . . 1

CHAPITRE I PRESENTATION DU SUJET ET CADRE GENERAL.

Introduction . . . . . . . . . . . . . . . . . . 6

1.1 Rappel historique ................ 6

1.2 Contenu problématique . . . . . . . . . . . 16

1.3 Mandat ................... . . 19

1.4 Méthodologie................... . . 20

1.5 Echéancier .. . . . . . . . . . . . 22

Conclusion . . . . . . . . . . . . . . . 23

\section{CHAPITRE II LA PROBLEMATIQUE}

Introduction . . . . . . . . . . . . . . 25

2.1 Description de I'environnemnt et/ou des

super-systèmes . . . . . . . . . . . . . . .

2.1.1 Le Point de services dans I'organisation systémique ............... 27

2.1.2 Environnement socio-géographique ....... . 28

2.1.3 Environnement financier........... 30

2.1.4 Environnement légal et physique......... 32 
2.1.4.1 Encadrement municipal . . . . . . . . 32

2.1.4.2 Encadrement légal provincial . . . . . . 33

2.1.4.3 Encadrement légal fédéral . . . . . . 34

2.1.4.4 Encadrement de régie interne

du Mouvement Desjardins . . . . . . . 34

2.2 Description des structures . . . . . . . . . . . 36

2.2.1 Organigramme de 1'institution . . . . . . . 37

2.2.2 Description des tâches . . . . . . . . . 39

2.2.2.1 Le directeur . . . . . . . . . . . 39

2.2.2.2 Le directeur-adjoint aux services
courants et administratifs . . . . . 40

2.2.2.3 Le directeur-adjoint aux services

2.2.2.4 La chef d'équipe . . . . . . . . . . . . . . . 43

2.2.2.5 Les caissières . . . . . . . . . 44

$\begin{array}{ll}2.2 .3 & \text { Les politiques opérationnelles et } \\ \text { règlements . . . . . . . . . . . . . . . } 44\end{array}$

2.2.3.1 Travail des caissières . . . . . . . 45

2.2.3.2 Rôle de I'adjoint courant et administratif . . . . . . . . . 45

2.2.3.3 Transport du numéraire ........ 45

2.2.4 Aménagement physique des lieux .......... 46

2.3 Description du processus .............. 49

2.3.1 Listes des opérations effectuées......... 49

2.3.2 Ordonnancement des opérations ......... 51

2.3.3 Opérations nécessaires pour effectuer

les transactions . . . . . . . . . . . 52

2.3.4 Opérations relatives au transport
du numéraire................... 53

2.4 Description du climat organisationnel . . . . . . . . 54

2.4.1 Le point de vue du directeuradjoint aux services courants et administratifs . . . . . . . . . . . 55

2.4.2 Le point de vue du directeur de la Caisse . . . . . . . . . . . . 57

2.4.3 Le point de vue des caissières . . . . . . . . 58 
2.5 Analyse des indicateurs . . . . . . . . . . 60

2.5.1 Les indicateurs financiers .......... 61

2.5.1.1 La marge brute ............ 62

2.5.1.2 Le trop-perçu net............ 64

2.5.1.3 Le bilan du Point de services....... 65

2.5.1.4 La croissance comparée de

I'épargne et du crédit . . . . . . . 67

2.5.2 Les indicateurs de marketing . . . . . . . 70

2.5.3 Les indicateurs de personnel . . . . . . . . 70

2.5.4 Les indicateurs de productivité . . . . . . . 71

2.6 Sélection d'une problématique à étudier . . . . . . . 73

2.6.1 Les situations problématiques . . . . . . . 73

2.6.1.1 L'achalandage ............ 73

2.6.1.2 Le marketing ............. . 74

2.6.1.3 La productivité ........... 75

2.6.2 La situation problématique retenue

pour étude . . . . . . . . . . . . . . 77

Conclusion . . . . . . . . . . . . . . . . 77

CHAPITRE III LE SYSTEME PERTINENT PROPOSE: UN SYSTEME DE PRODUCTION

Introduction . . . . . . . . . . . . . . . .

3.1 Le choix du système pertinent . . . . . . . . . 79

3.1.1 Quel système adopter? ............. 79

3.1.2 Justification d'un tel système . . . . . . . 80

3.2 Revue de Littérature:

Les coopératives d'épargne et de crédit

dans notre milieu. . . . . . . . . . . . . .

3.2.1 Les caisses populaires et le

développement régional au

Saguenay-Lac-Saint-Jean . . . . . . . . . . 85

3.2.2 Le rôle social des caisses

populaires Desjardins . . . . . . . . . . 95

3.2.3 Comment la caisse réalise son

action socio-économique ........... 102 
3.2.3.1 L'argent au service de

I'humain ............. . 103

3.2.3.2 L'engagement personne1......... 104

3.2.3.3 L'action démocratique ......... 104

3.2.3.4 L'intégrité et la rigueur dans 1 'entreprise coopérative . . . . 105

3.2.3.5 La solidarité avec le milieu . . . . . 106

3.3 Le système pertinent . . . . . . . . . . 107

3.3.1 Définition du système pertinent......... 107

3.3.2 Validation de la définition .......... 108

Conclusion ................... 110

CHAPITRE IV CONCEPTUALISATION DE LA SOLUTION RETENUE

Introduction ................ 113

4.1 Le modèle conceptuel . . . . . . . . . . . 114

4.1.1 Système général des opérations

du Point de services ........... 115

4.1 .2 Les sous-systèmes d'activités ......... 116

4.1.2.1 Les préparatifs d'ouverture

du Point de services........ 116

4.1.2.2 Les transactions courantes ........ 118

4.1.2.3 La négociation des placements

et des prêts ............ 119

4.1.2.4 La réception du numéraire ....... 120

4.2 Validation du modèle conceptuel ......... 121

4.3 Comparaison avec la réalité . . . . . . . . 123

Conclusion . . . . . . . . . . . 125

CHAPITRE V IDENTIFICATION DES CHANGEMENTS POSSIBLES

Introduction . . . . . . . . . . . . . 127

5.1 Les changements dus au modèle proposé . . . . . . 128

5.1.1 Aménagement des espaces physiques . . . . . . 128

5.1.1.1 Aménagement intérieur . . . . . . 129

5.1.1.2 Aménagement extérieur ........ 131 
5.1.2 Modifications aux ressources humaines . . . . 131

5.1.2.1 Création d'un nouveau poste ...... 131

5.1.2.2 Le travail de caissière . . . . . . 133

5.1.3 Modifications de nature fonctionnelle
et décisionnelle . . . . . . . . . 133

5.1.3.1 Modification de nature
fonctionnelle ............ 133

5.1.3.2 Modification à la structure
décisionnelle .......... . . 133

5.1.4 Coûts approximatifs des changements ...... 135

5.1.4.1 Coûts des rénovations ......... 135

5.1.4.2 Coûts de $1^{\prime}$ embauche de
personnel ................... 135

5.2 Une solution alternative . . . . . . . . . . 136

Conclusion . . . . . . . . . . . . . . 139

\section{CHAPITRE VI REFLEXION METHODOLOGIQUE}

Introduction ................... 141

6.1 La méthode.................. 141

6.1.1 Qualités de la méthode ............. 144

6.1.2 Risques et dangers de la méthode . . . . . . 145

6.2 La démarche . . . . . . . . . . . . . 146

6.3 Les connaissances acquises ............. 147

Conclusion . . . . . . . . . . . . . . . 148

CONCLUSION • . . . . . . . . . . . . . . . . . . . 149

BIBLIOGRAPHIE . . . . . . . . . . . . . . . . 152

ANNEXES . . . . . . . . . . . . . . . . . . 156

1. Dirigeants fondateurs de la caisse populaire

Saint-Jean-Eudes . . . . . . . . . . . . 157

2. Etats financiers, St-Jean-Eudes (1973) . . . . . . 158

3. Etats financiers, Arvida (1973) ........... 161

4. Bilan consolidé au 31 mai 1973 . . . . . . . . . . 165

5. Sondage . . . . . . . . . . . . . . 167 
1- Point de services:

L'expression Point de services écrite dans le texte avec une lettre majuscule au début signifie: le Point de services de Saint-Jean-Eudes.

\section{2- Caisse}

Le mot Caisse écrit dans le texte avec une majuscule signifie: la Caisse populaire d'Arvida.

\section{3- Fédération}

Le mot Fédération écrit dans le texte avec une majuscule signifie la Fédération des caisses populaires Desjardins du Saguenay-Lac-Saint-Jean.

N.B Ces précisions n'excluent pas la possibilité de retrouver la désignation complète de l'entité plutôt que son abrégé.

4- Prêts R/D

Dans le texte et surtout dans les tableaux, cette abréviation signifie: prêts sur reconnaissance de dettes.

5- EOP

Dans le texte et surtout dans les tableaux, cette abréviation signifie: I'épargne avec opérations. 
6- /100 P.d.

Dans les tableaux, cette abréviation signifie: par $100 \$$ de passif-dépôt.

7- Adjoint conseil

Synonyme de directeur-adjoint aux services conseil.

8- Adjoint courant et administratif

Synonyme de directeur-adjoint aux services courants et administratifs.

9- H.C.P.E.D.

Mouvements des caisses populaires et d'économie Desjardins.

10- C.A.

Dans le texte, ces deux lettres en majuscule signifient:

conseil d'administration. 
$\underline{\text { Page }}$

TABLEAU I Caisse populaire de Saint-Jean-Eudes, Etat de l'actif et du passif au 31 mai 1951.

TABLEAU II Caisse populaire de Saint-Jean-Eudes, Progression des épargnes et du crédit, de la Fondation à la fusion.

TABLEAU III Evolution de I'Epargne (1982 - 1985), Point de services et siège social.

TABLEAU IV Evolution du Crédit (1982 - 1985), Point de services et siège social.

TABLEAU V La marge brute, Point de services 31 juillet 1986

TABLEAU VI Trop-perçu net, Point de services

31 juillet 1986

TABLEAU VII Point de services, Bilan pro-forma au 18 juillet 1986

TABLEAU VIII Le Point de services par rapport au siège social, la Caisse populaire d'Arvida, les Caisses et Banques au Québec (1982 et 1984)

TABLEAU IX Croissance de population, paroisse Saint-Jean-Eudes.

TABLEAU $X$ Taux de croissance du nombre de sociétaires, Point de services

TABLEAU XI Population du Saguenay-Lac-Saint-Jean

TABLEAU XII Taux de pénétration des caisses populaires, Fédération du Saguenay-Lac-Saint-Jean et Confédération.

TABLEAU XIII Répartition des caisses par municipalité selon l'importance de leur population au Saguenay-Lac-Saint-Jean. 


\section{LISTE DES FIGURES}

Page

$\begin{array}{llll}\text { FIGURE } & \text { I } & \text { L'organisation systémique } & 27\end{array}$

FIGURE II Organigramme, Caisse populaire d'Arvida 38

FIGURE III Aménagement intérieur, Point de services 47

FIGURE IV Stationnements au Point de services $\quad 48$

$\begin{array}{llll}\text { FIGURE } \quad V \text { Schéma des opérations } & 51\end{array}$

$\begin{array}{llll}\text { FIGURE } & \text { VI Procédures d'une transaction } & 52\end{array}$

FIGURE VII Réception du numéraire

FIGURE VIII Histogramme, Caisses et Banques, Région 0294

FIGURE IX Système des opérations du Point de services 115

$\begin{array}{llll}\text { FIGURE } X \text { Sous-système: } & 117\end{array}$

FIGURE XI Sous-système: Effectuer les transactions $\quad$ 118

FIGURE XII Sous-système: Négocier Placement/Prêt 119

FIGURE XIII Sous-système: Recevoir le numéraire 120

FIGURE XIV Réaménagement intérieur du Point de 130

$\begin{array}{llll}\text { FIGURE } & \text { XV } & \text { Organigramme du Point de services } & 134\end{array}$

$\begin{array}{llll}\text { FIGURE XVI Processus d'ouverture du Point de } & 138\end{array}$ services: solution alternative 
INTRODUCTION 


\section{INTRODUCTION}

En février 1985, c'était une première participation à une assemblée générale de la Caisse populaire d'Arvida en tant que membre du conseil d'administration. Professeur en éducation économique au niveau $V$ du cours secondaire général, les mécanismes de fonctionnement d'une caisse populaire nous étaient connus pour les avoir expliqués aux étudiants. Mais là, nous vivions pleinement depuis une année les rouages administratifs de la Caisse populaire d'Arvida. Au cours de la douzaine de réunions que nous avons tenues cette année-là, nous avons acquis peu et beaucoup de choses en même temps. En effet, à chaque assemblée, beaucoup de décisions devaient se prendre; et ces décisions demandaient parfois que chaque membre du conseil d'administration consulte et mâ̂trise une quantité parfois importante de documents de toute sorte. Donc, après chacune de nos rencontres, des connaissances plus élaborées sur le système financier, le budget, la gestion du crédit, des taux d'intérêt, la gestion des resssources humaines... et ausssi sur l'action sociale en milieu coopératif garnissaient notre savoir, notre savoir-faire ou notre savoir-être.

II est arrivé en maintes occasions, pendant nos réunions, que nous ayons à discuter de certains sujets concernant le Point de services de la Caisse à Saint-Jean-Eudes. Entre autres choses, plusieurs plaintes étaient parvenues au conseil d'administration à cause de billets d'infraction pour stationnement interdit. En quelques occasions, il a été question d'achat de l'immeuble du Point de services propriété de Ville de Jonquière, ou tout simplement de sa relocalisation; surtout 
depuis que la ville avait procédé à la réfection du Boulevard Saguenay en l'élargissant et y ajoutant un terre-plein au centre. Une des décisions prises au C.A. avait été de mandater le comité de l'immeuble pour qu'il puisse élaborer un projet de relocalisation du Point de services. Il était hors de question que la Caisse acquiert l'immeuble du Point de services; en plus, Ville de Jonquière avait avisé qu'elle mettrait prochainement un terme au bail liant les deux parties, soit en mai 1986.

Au terme de cette assemblée générale de février 1985, faisant part au directeur de la Caisse des difficultés à trouver une PME pour en faire l'étude dans une recherche-action, celui-ci suggéra de rédiger ce mémoire de mâ̂trise sur la valeur et l'importance du Point de services de la Caisse à Saint-Jean-Eudes. Plusieurs éléments, d'une problématique intéressante avaient été soulevés dans des réunions antérieures du C.A.. Le directeur fournissait également certaines balises autour desquelles devrait-on axer notre recherche et notre action. Le Point de services fait partie intégrante de la communauté de Saint-Jean-Eudes. Le fermer constituerait une erreur grave de la Caisse et entraînerait une perte financière importante.

Un projet sommaire comportant certains échéanciers a donc été préparé et soumis aux administrateurs pour approbation ou refus. Le projet accepté, I'étude du Point de services commençait et un plan précis de travail a été conçu.

Notre travail sera donc divisé en six étapes importantes qui amèneront le lecteur, le décideur, à prendre connaissance du sujet de la 
problématique à laquelle il est confronté et de la solution proposée. Notre étude débutera donc par une présentation du sujet et une définition du cadre général de travail. Dans un deuxième temps, une analyse de I'environnement et de toutes les structures, organisationnelles, fonctionnelles et opérationnelles du Point de services nous aidera à établir une problématique à solutionner. Le troisième chapître établira les bases de la solution proposée, le tout étant appuyé par une revue de littérature sur le rôle socio-économique des caisses populaires Desjardins au Saguenay-Lac-Saint-Jean.

Le chapitre IV permettra de construire un modèle conceptuel de la solution proposée alors que le chapître $V$ identifiera les changements possibles à envisager pour opérationnaliser la solution. Enfin, nous réfléchirons sur la méthode de travail utilisée pour traiter de l'importance de maintenir ouvert le Point de services de la Caisse populaire d'Arvida à Saint-Jean-Eudes. 
CHAPITRE I

PRESENTATION DU SUJET ET CADRE GENERAL 


\section{CHAP ITRE I}

PRESENTATION DU SUJET ET CADRE GENERAL

INTRODUCTION

Lorsqu'on veut concevoir une solution à une problématique qui nous est présentée, il convient d'en rechercher toutes les composantes. La dynamique d'une solution dans l'action nous commande donc de ne négliger aucun élément passé ou présent qui pourrait avoir une incidence dans le futur.

Dans le cas du Point de services de Saint-Jean-Eudes, il nous apparaissait comme primordial de souligner le caractère d'autonomie de fonctionnement qui a marqué sa naissance. Ainsi, une certaine logique nous obligeait à relater certaines pages de l'histoire du Point de services.

Le rappel historique qui suit vise donc à situer dans le temps et les faits l'évolution du Point de services, nous permettant par la suite de justifier une partie de la solution que nous pourrions apporter.

\section{I.I RAPPEL HISTORIQUE}

En 1950, les habitants de la paroisse Saint-Jean-Eudes du Canton de Chicoutimi, qui sont alors au nombre de 3500 environ, ne sont desservis par aucune institution financière. La population doit donc parcourir de cinq à six kilomètres et se rendre en la ville d'Arvida pour profiter des différents services bancaires qui y sont offerts. 
Vigoureusement appuyé par le curé de la paroisse, Monsieur I'abbé Marcel Gauthier, un groupe de citoyens, ayant à sa tête monsieur Marcellin McNicoll, lance l'idée qu'il serait possible d'ouvrir une caisse populaire pour desservir la population du milieu. Aidé de monsieur l'abbé Gérard Lévesque, aumônier diocésain des caisses populaires Desjardins, on entreprend des démarches auprès du siège social du Mouvement Desjardins à Lévis afin d'obtenir les renseignements et conditions nécessaires à l'implantation d'une telle institution dans la paroisse. La date du 7 septembre 1950 est donc retenue par le groupe de citoyens pour tenir une assemblée de fondation d'une caisse populaire à Saint-Jean-Eudes. L'annexe I nous présente la liste des premiers dirigeants élus ce 7 de septembre.

Aux dires des administrateurs de la nouvelle caisse et selon l'aumonier diocésain des caisses populaires, le démarrage de I'institution financière fut considéré comme un succès. En effet, les rapports financiers du premier secrétaire-gérant, monsieur Léonce Bilodeau, révèlent des dépôts d'épargne de 14678,40 \$ et des prêts pour un montant de 1223 \$ environ: Le tableau I ci-après fournit plus de détails sur la situation financière de la caisse à la fin de la première année sociale, le 31 mai 1951, laquelle année fut d'une durée de neuf mois seulement. 
TABLEAU I

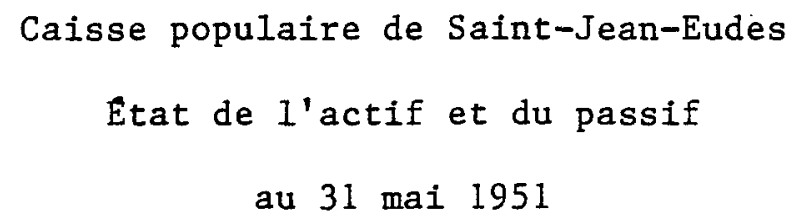

$\overline{\text { Actif }}$

Encaisse

Placements

Prêts aux sociétaires

Immobilisations (inventaires)

Grand total de l'actif
$11263,97 \$$

2031,81

13295,78

$13295,78 \$$

1223,00

206,25

14725,03

$\overline{\text { Passif }}$

Capital social

Epargne
1802,95

12875,45
14678,40

Autres éléments du passif

- Fonds de la réserve générale 45,40

- Salaire dû au gérant $\quad .1,00$

- Surplus

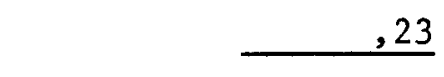

Tota1 46,63\$ 46,63

Grand total du passif $14725,03 \$$

Source: Procès-verbal, Assemblée générale Caisse populaire Saint-Jean-Eudes, 5 juillet 1951. 
Démarrer une institution de services bancaires du type coopérative d'épargne et de crédit nécessite une grande participation populaire et beaucoup de bénévolat. A la caisse populaire de SaintJean-Eudes, le bénévolat s'est étendu jusqu'à la fonction de gérant. Monsieur Léonce Bilodeau, le secrétaire-gérant, dirigeait les opérations de la caisse à partir de chez lui pour la modique somme de $1 \$$. Cette rémunération de 1 \$ pour 12 mois avait été votée par le conseil d'administration à $I^{\prime}$ assemblée du 7 septembre 1950 .

Plusieurs faits importants ont marqué les cinq premières années d'existence de la Calsse populaire de Sain-Jean-Eudes. Il y avait d'abord l'omniprésence du clergé paroissial dans l'administration de la caisse. Le curé était de toutes les assemblées générales et parfois de celles du conseil d'administration. Il présidait toujours (ou presque) les élections des dirigeants; et, à la fin des assemblées générales,. il prenait la parole pour apporter: commentaires, incitations et/ou directives. Le curé de I'époque, l'abbé Marcel Gauthier était membre honoraire du conseil d'administration.

Le deuxième élément marquant ces premiers cinq ans est I'orientation économique de la caisse qui est fortement axée sur la recherche de 1'épargne. Les états financiers de ces années démontrent bien cette tendance; à $1 a$ fin de l'année sociale de 1956 on avait accumulé pour plus de $60000 \$$ en épargne alors que les prêts consentis ne représentaient guère plus que $33 \%$ de la valeur de l'épargne. Chaque prêt sur reconnaissance de dettes ne pouvait dépasser les $500 \$$. Et il a 


\begin{abstract}
fallu attendre l'année 1956 pour enregistrer les premiers prêts hypothécaires. Le tableau II de la page suivante nous montre bien cette tendance.
\end{abstract}


TABLEAU II

Caisse populaire de St-Jean-Eudes

Progression des épargnes et du crédit

De la fondation à la fusion

\begin{tabular}{|c|c|c|c|c|c|c|c|c|}
\hline DATES $^{(I)}$ & & รOTTS & & & & PRẼTS & & \\
\hline & Parts sociales & $\%(2)$ & Epargnes & $\%^{(2)}$ & R. de dettes & $\%(2)$ & Hypothécaires & $\%(2)$ \\
\hline $51-08-13$ & 1941.46 & & 16887.30 & & 1852.00 & & & \\
\hline $53-04-20$ & 2431.45 & 25.2 & 25708.43 & 52.2 & 9462.74 & -110.9 & & \\
\hline $54-03-30$ & 3134.94 & 28.9 & 31144.61 & 21.1 & 9377.04 & $-\quad .09$ & & \\
\hline $55-04-18$ & 3566.75 & 13.8 & 47321.53 & 51.9 & $15 \quad 252.52$ & 62.6 & & \\
\hline $56-05-22$ & 3992.69 & 11.9 & $55 \quad 435.25$ & 17.1 & $16 \quad 159.00$ & 5.9 & 4260.00 & \\
\hline $57-04-01$ & 4128.30 & 3.8 & 77890.92 & 40.5 & 15467.93 & -4.3 & 22690.00 & 432.6 \\
\hline $58-04-22$ & 4193.04 & 1.6 & 97972.31 & 25.8 & $12 \quad 112.95$ & -21.7 & $28 \quad 090.36$ & 23.8 \\
\hline $59-05-19$ & 4147.21 & -1.1 & $98 \quad 353.05$ & 0.4 & 11551.90 & -4.6 & 43088.61 & 53.4 \\
\hline $60-03-28$ & 3979.18 & -4.1 & 85636.16 & -12.9 & 8742.69 & -24.3 & $40 \quad 086.02$ & -7 \\
\hline $61-03-01$ & 3643.96 & -8.4 & 89977.08 & 5.1 & 5386.66 & -38.4 & 31330.30 & -21.9 \\
\hline $63-02-19$ & 3534.33 & -3 & 107359.70 & 19.3 & 7604.34 & 41.2 & 29686.92 & -5.2 \\
\hline $64-07-13$ & 4039.66 & 14.2 & 172346.21 & 60.6 & 24307.49 & 219.7 & 69929.96 & 135.6 \\
\hline $65-07-22$ & 4249.81 & 5.2 & 162466.62 & -5.7 & $25 \quad 263.74$ & 3.9 & 74135.04 & 6. \\
\hline $68-09-17$ & 9316.21 & 119.2 & 237082.45 & 45.8 & 46317.79 & 174.4 & 57890.74 & -21.9 \\
\hline $69-06-10$ & 8652.21 & -7.1 & $247 \quad 652.31$ & 4.5 & 39013.51 & -15.8 & 53629.42 & -7.4 \\
\hline $73-05-3 i$ & 7541.00 & -12.8 & 406890.00 & 64.3 & $98 \quad 935.00$ & 153.6 & 31793.00 & -40.7 \\
\hline
\end{tabular}

Source: Archives de la Caisse populaire d'Arvida, Rapports d'inspection de l'Union Régionale des Caisses populaires Desjardins du Saguenay-Lac-St-Jean.

Notes: Voir page suivante. 
Note 1: a) Les dates indiquées représentent celles des rapports d'inspection de la Caisse par la Fédération des Caisses populaires.

b) Les données des années 1952, 1962, 1966, 1967, 1970, 1971, 1972 n'étaient plus disponibles.

c) Les données du 31 mai 1973 sont celles reproduites au bilan précédant la fusion avec la Caisse populaire d'Arvida.

Note 2: Les pourcentages représentent les écarts entre les données de sa ligne et la ligne précédente. 
Les extraits suivants de différents procès-verbaux illustrent bien l'intime relation entre les deux faits précédents.

“Je demande à chacun de vous d'être des apôtres de l'épargne. C'est en épargnant que nous pourrons atteindre les plus hauts sommets." disait le curé, monsieur Joseph Lavoie.

"Je suis certain, si nous le voulons, que nous pourrons être 700 sociétaires et beaucoup plus d'épargnants, car il y a beaucoup d'argent à droite et à gauche, qui devrait être à la caisse afin de servir à aider les gens de Saint-Jean-Eudes" ${ }^{2}$ disait encore le même curé.

Le point le plus important caractérisant cette institution financière à ses débuts, apparaît comme étant la grande fragilité de son organisation opérationnelle et administrative. En effet, durant ces premières années, les administrateurs durent remplacer un ou plusieurs dirigeants démissionnaires. Chacun des trois conseils eut à subir au moins une démission. Au cours de l'assemblée générale annuelle du 30 juillet 1953, le curé de la paroisse soulignait le manque d'intérêt des sociétaires envers leur caisse à cause de leur très faible participation.

1- Intervention du curé, monsieur Joseph Lavoie. Extrait du procès-verbal de 1'assemblée générale de la Caisse populaire de Saint-Jean-Eudes, 13 avri1 1952 .

2- Intervention du curé, monsieur Joseph Lavoie. Extrait du procès-verbal de l'assemblée gérérale de la Caisse populaire de Saint-Jean-Eudes. Le 30 juin 1954. 
De plus, il notait la grande faiblesse de l'épargne qui selon lui aurait dû atteindre à cette date une somme supérieure à 100000 \$. Cette absence d'épargne ajoutait à la fragilité administrative car elle ne permettait pas aux gérants et officiers de consentir à tous les prêts sollicités par les gens du milieu. Monsieur Marcellin McNicoll, membre de la première commission de surveillance en 1950 et encore administrateur en 1985, expliquait en ces termes les démissions fréquentes des premières années: "Les gens des différents conseils connaissaient très peu les mécanismes du système coopératif et avaient souvent l'impression qu'on les faisait travailler pour rien." 3

Grâce à la ténacité des dirigeants, la caisse finit par atteindre une stature intéressante, compte tenu de son nombre de déposants et du bassin de population qu'elle desservait. Monsieur Léonce Bilodeau cumula pendant longtemps les fonctions de secrétaire du conseil d'administration et de gérant de la caisse; cela pour un revenu très modeste. Monsieur Charles-Eugène Ratthé, le président fondateur n'a jamais ménagé son temps et ses efforts pour conduire sur la bonne voie I'entreprise qu'il dirigeait. Quant à monsieur Pierre Roy, qui fut du premier conseil d'administration, il devint président dans les années 1960; et plus souvent qu'à son tour, il eut à assumer en plus la fonction de gérant, pour un dollar (1\$) seulement, parce que beaucoup de gérants

3- Entretien de 1'auteur avec M. McNicoll en mai 1986. 
remettaient, leur démission. Tout cela se déroula si bien que, quelque vingt ans après la fondation, on parlait de dépôts de l'ordre de 200000 \$ ou plus.

Malgré tout cela, on sentait le besoin d'améliorer la qualité et la valeur des services. Mais on atteignait une certaine limite à cause de la faible densité de population du secteur. C'est donc à ce moment-là que naît l'idée d'une fusion possible avec la Caisse populaire d'Arvida. Comme la municipalité de Saint-Jean-Eudes venait à peine de s'unir à la Ville d'Arvida, l'idée s'avère donc intéressante et fait rapidement son chemin. De cette façon on espérait améliorer les services existants et en ajouter d'autres.

Le 26 juin 1973, sur une proposition de monsieur Denis Deschamplain, appuyée par monsieur Charles-Eugène Ratté, il est résolu unanimement que la Caisse populaire de Saint-Jean-Eudes devienne un point de services de la Caisse populaire d'Arvida. ${ }^{4}$ Les états financiers de la Caisse de Saint-Jean-Eudes laissaient voir un actif total de 423135 . La valeur des dépôts d'épargne dépassait les 400000 \$. Quant aux différents prêts, ils représentaient une somme supérieure à 120000 . Les annexes II, III, et IV illustrent la situation financière de chacune des deux caisses qui se fusionnaient et la situation de la nouvelle caisse ainsi constituée.

4- Procès-verbal de l'assemblée générale spéciale de la Caisse populaire de St-Jean-Eudes, le 26 juin 1973, Archives de la Caisse populaire d'Arvida 
Dix ans plus tard, l'épargne globale des gens du secteur desservi par le Point de services totalisait 2006676 \$. Les prêts sur reconnaissance de dettes ainsi que les prêts hypothécaires se chiffraient à 1716931 \$. Au moment de la fusion, on espérait une amélioration sensible de la qualité et de la quantité des services offerts. La croissance de la valeur de I'épargne et des prêts traduit-elle cette amélioration désirée par les sociétaires de Saint-Jean-Eudes?

\subsection{CONTENU PROBLEMATIQUE}

Au cours des 4 dernières années (1982-1985), le directeur de la Caisse populaire d'Arvida a constaté une diminution sensible du nombre de comptes-clients; ce nombre passant de 1552 à 1 092, soit une diminution de $29,64 \%$. De plus, quelques uns de ces comptes semblent inactifs ou presque, car seulement 1036 sur 1092 utilisent le compte d'épargne avec opération. Pourtant, durant la même période, I'épargne totale des sociétaires c'est accrue de 437947 \$ pour une augmentation de 23,6\%. Pour leur part, les prêts sur reconnaissance de dettes et les prêts hypothécaires ont vu leur valeur croître de 508178 \$ pour une hausse de 29,6\%. Les tableaux III et IV des deux pages suivantes nous présentent une évolution plus détaillée de l'épargne et du crédit tant au Point de services qu'à la Caisse sur le boulevard Mellon. 
TABLEAU III

EVOLUTION DE L'EPARGNE

POINT DE SERVICES ET SIEGE SOCIAI

CAISSE POPULAIRE D'ARVIDA

$1982-1985$

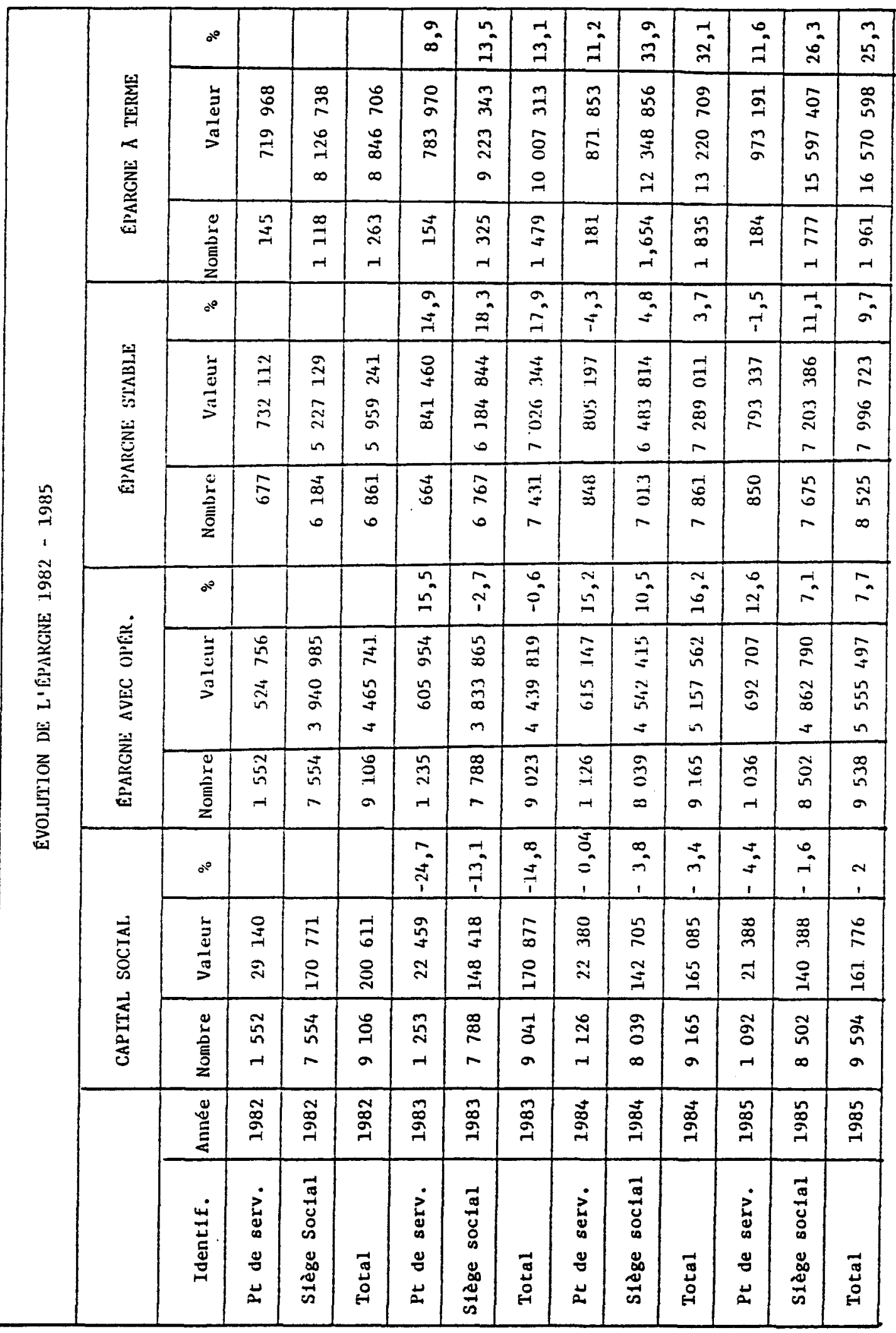


TABLEAU IV

E์VLUTION DU CREDIT 1982 - 1985

\begin{tabular}{|c|c|c|c|c|c|c|c|}
\hline & & Reconn & ssance des $d$ & ttes & & Bypothécaire & \\
\hline Identification & Année & Nombre & Valeur & $\%$ & Nombre & Valeur & $\because$ \\
\hline Pt de service & 1982 & 280 & 861447 & & 54 & 855484 & \\
\hline Siège social & 1982 & 1382 & $\begin{array}{lll}5 & 513 & 163\end{array}$ & & 444 & 8965380 & \\
\hline Total & 1982 & 1662 & $6374 \quad 610$ & & 498 & 9820864 & \\
\hline Pt de service & 1983 & 253 & $730 \quad 371$ & $-15,2$ & 55 & 911746 & 6,6 \\
\hline Siège social & 1983 & 1408 & 7457402 & 35,3 & 443 & 10137360 & 13,1 \\
\hline Total & 1983 & 1661 & 8187773 & 28,4 & 498 & 11049106 & 12,5 \\
\hline Pt de service & 1984 & 252 & 767501 & 5,1 & 54 & 1110103 & 21,8 \\
\hline Siège social & 1984 & 1542 & 8535001 & 14,5 & 483 & $12453 \quad 249$ & 22,9 \\
\hline Total & 1984 & 1794 & 9302502 & 13,6 & 537 & $13 \quad 563 \quad 352$ & 22,8 \\
\hline Pt de service & 1985 & 256 & 877855 & 14,4 & 56 & 1377254 & 24,1 \\
\hline Siège social & 1985 & 1911 & 11411725 & 33,7 & 460 & 12622388 & 1,4 \\
\hline Total & 1985 & 2167 & $12 \quad 289580$ & 32,1 & 516 & 13999642 & 3,2 \\
\hline
\end{tabular}


On est donc porté à se demander chez le personnel cadre de la Caisse, si le Point de services n'aurait pas perdu plusieurs de ses clients au profit d'une autre institution bancaire. En fait, pour le directeur de la Caisse, la question à se poser est la suivante: Quelle est l'importance économique et sociale du Point de services de Saint-Jean-Eudes? Faut-il envisager sa fermeture à moyen terme ou bien une réorganisation physique et/ou administrative?

Quelques plaintes ont déjà été formulées au Conseil d'administration concernant les difficultés de stationnement depuis la réfection du boulevard Saguenay. De plus, les services spécialisés d'épargne et de crédit ont été centralisés au siège social de la Caisse populaire d'Arvida sur le Boulevard Mellon à Jonquière. Et cela peut paraittre une diminution importante dans la qualité des services offerts à la population.

\subsection{MANDAT}

Après avoir discuté quelque peu du sujet avec lui, il fut convenu que le directeur présenterait au Conseil d'Administration une proposition d'étude sur ce contenu problématique du Point de services de Saint-Jean-Eudes. Le Conseil d'Administration accorda le mandat sans hésitation. Etant également membre de ce conseil d'administration, nous avons présenté un plan plus détaillé du projet d'étude lors d'une réunion subséquente. 
Avec ce projet, on espère principalement améliorer la qualité des services offerts. On vise en outre à recruter une nouvelle clientèle qui viendrait, par ses dépôts, accrỗtre le potentiel d'affaires du Point de services. Enfin, on souhaite attirer à la Caisse plusieurs comptes commerciaux, ce qui fait passablement défaut à Saint-Jean-Eudes.

\subsection{MÉTHODOLOGIE}

Nous entendons réaliser cette étude en utiliant comme technique de base une méthodologie de diagnostic organisationnel: "Le diagnostic intervention». Monsieur Paul Prévost, Docteur en sciences de administration, maintenant à l'Université de Sherbrooke, a fait cette adaptation de la théorie de l'approche systémique conçue par P.B. Checkland de 1'Université Lancaster, Angleterre. Parce que, dans cette méthode, l'organisation est considérée comme un ensemble d'activités humaines interreliées par des flux de personnel, d'argent, d'informations, "le diagnostic intervention" nous apparaît d'une souplesse suffisante pour permettre l'analyse de situations complexes.

La structure d'analyse de la méthode de Paul Prévost comprend les sept volets de travail suivants:

1- Elaboration d'une problématique.

2- Point d'ancrage.

3- Conceptualisation.

4- Comparaison avec la réalité.

5- Identification des changements possibles.

6- Applications des changements sélectionnés.

7- Contrôle 
Dans la présente recherche, nous avons privilégié I'utilisation des cinq premiers volets seulement. La raison principale qui motive cette façon de faire s'explique par le fait que, pour le directeur et les dirigeants auxquels nous avons soumis le projet de recherche, la priorité première consiste en la rénovation du siège social de la Caisse. Le Point de services représente plutôt un projet à plus long terme.

De plus, cette méthode comporte l'avantage de recourir à I'implication de plusieurs personnes. C'est ainsi que la collaboration et les connaissances du directeur ont été grandement sollicitées. Il en fut de même pour les autres membres du personnel cadre et de quelques employées. Grâce à cette collaboration nous avons pu obtenir et consulter toutes les données financières pertinentes et nécessaires pour la confection des tableaux statistiques utilisés. Il en fut de même pour plusieurs autres documents d'archive essentiels à la rédaction de la partie historique.

Comme le sociétaire constitue l'acteur principal dans le fonctionnement d'une caisse, les membres du Point de services ont été consultés par le biais d'un sondage effectué au cours de l'été 1985. Ce sondage nous a permis d'établir un certain profil de la clientèle, à partir duquel nous avons pu dégager certains éléments de la problématique, pour ensuite s'en servir dans l'élaboration d'une solution possible. A I'annexe $V$ nous pouvons consulter ce questionnaire sondage et vérifier les résultats obtenus. 
Enfin l'évolution de la philosophie coopérative a tellement été importante au cours des dernières décennies qu'il aura fallu effectuer une revue de certains écrits récents et plus anciens pour justifier une partie de la solution à retenir.

Somme toute, la dynamique de cette méthode de recherche-action oblige la participation de tous les niveaux décisionnels; tant celui des propriétaires-sociétaires que celui des dirigeants et celui des personnels. Et une telle façon de procéder demande passablement de temps.

\subsection{ECHEANCIER}

Deux paramètres ont donc servi à étaler dans le temps la réalisation de ce projet de recherche. Il y a d'abord les besoins de la Caisse. Ceux-ci n'étant pas prioritaires et urgents, il ne fut imposé aucun délai pour le dépôt du rapport final. Des comptes-rendus périodiques s'avéraient suffisants pour permettre aux dirigeants de prendre connaissance de la progression de l'étude.

La deuxième contrainte de temps provenait des exigences universitaires. Celles-ci nous obligeaient à déposer la recherche à la fin de l'été 1985. Toutes sortes de circonstances incontrôlables, telle la maladie, ont fait qu'une deuxième prolongation d'une année s'est avérée nécessaire pour permettre à l'auteur de satisfaire à toutes les exigences d'une telle recherche. 
CONCLUSION

Dans ce présent chapître, nous avons essayé de définir un cadre méthodologique à l'intérieur duquel nous entendons procéder. Les quelques pages qui composent le rappel historique et qui ont été placées en début de chapître, se veulent le cadre de référence à partir duquel nous espérons dégager, au cours du prochain chapître, toute la problématique qu'à pu engendrer le fait de passer de caisse autonome à dépendance d'une autre comme point de services. Il est bien évident que ce changement dans l'histoire de la Caisse populaire de Saint-Jean-Eudes ne constitue pas la seule raison qui a amené le directeur à s'interroger sur sa valeur. Cependant nous nous accordons pour affirmer que cela représente le principal phénomène déclencheur de la problématique que nous tenterons de construire au chapitre suivant. 
CHAPITRE II

LA PROBLEMATIQUE 


\section{CHAPITRE II}

LA PROBLEMATIQUE

INTRODUCTION

Entre 1982 et 1985, le nombre de sociétaires du Point de services chutait d'environ $30 \%$. Une telle diminution ne déclenche-t-elle pas une sonnerie d'alarme au sein de l'organisation administrative de l'entreprise qui la subit? Force nous est d'admettre son importance et son danger. Mais avant d'entreprendre quoi que ce soit pour enrayer le malaise, il importe d'en analyser les composantes, en découvrir les principaux symptômes, pour ensuite, y appliquer les remèdes nécessaires.

$\mathrm{Au}$ cours de ce chapître qui s'amorce, notre travail consistera donc à rechercher tous les faits et renseignements permettant d'identifier les causes de la faiblesse pour porter par la suite un diagnostic reconnaissant le plus exactement possible le problème à solutionner. Pour y arriver, nous allons d'abord situer le Point de services dans son environnement social, géographique, financier, politique et légal.

Dans un deuxième temps, le Point de services sera décrit dans ses structures organisationnelles et physiques. Par après nous procéderons à la description de son processus opérationnel et de son climat organisationne1. 
Finalement, nous ferons une analyse exhaustive des principaux indicateurs financiers, de personnel, de marketing et de productivité. De cette étude, nous déduirons quels sont les problèmes auxquels le Point de services est confronté. Et parmi ces derniers, nous choisirons la problématique qui nous semble la plus importante à résoudre dans l'immédiat.

\subsection{DESCRIPTION DE L'ENVIRONNEMENT ET/OU DES SUPER-SYSTEMES}

Décrire, sous plusieurs angles, l'environnement d'un système permet aux lecteurs et surtout aux preneurs de décisions, de mieux le situer dans toute I'organisation systémique. A Saint-Jean-Eudes, le Point de services, érigé en système autonome, est devenu, avec les années et pour diverses raisons déjà relatées dans le chapître précédent, un sous-système de la Caisse populaire d'Arvida.

A la lecture des pages suivantes, décrivant 1'environnement social, géographique, financier et légal, nous pourrons découvrir que le Point de services dessert, seul, une collectivité isolée géographiquement et financièrement des deux grands centres urbains (soient Jonquière et Chicoutimi); et cela, même si la population de Saint-Jean-Eudes fait partie démographiquement de ces deux villes.

Quant à la figure I qui suit immédiatement elle trace le portrait schématique de 1'organisation systémique dans laquelle opère le Point de services. 


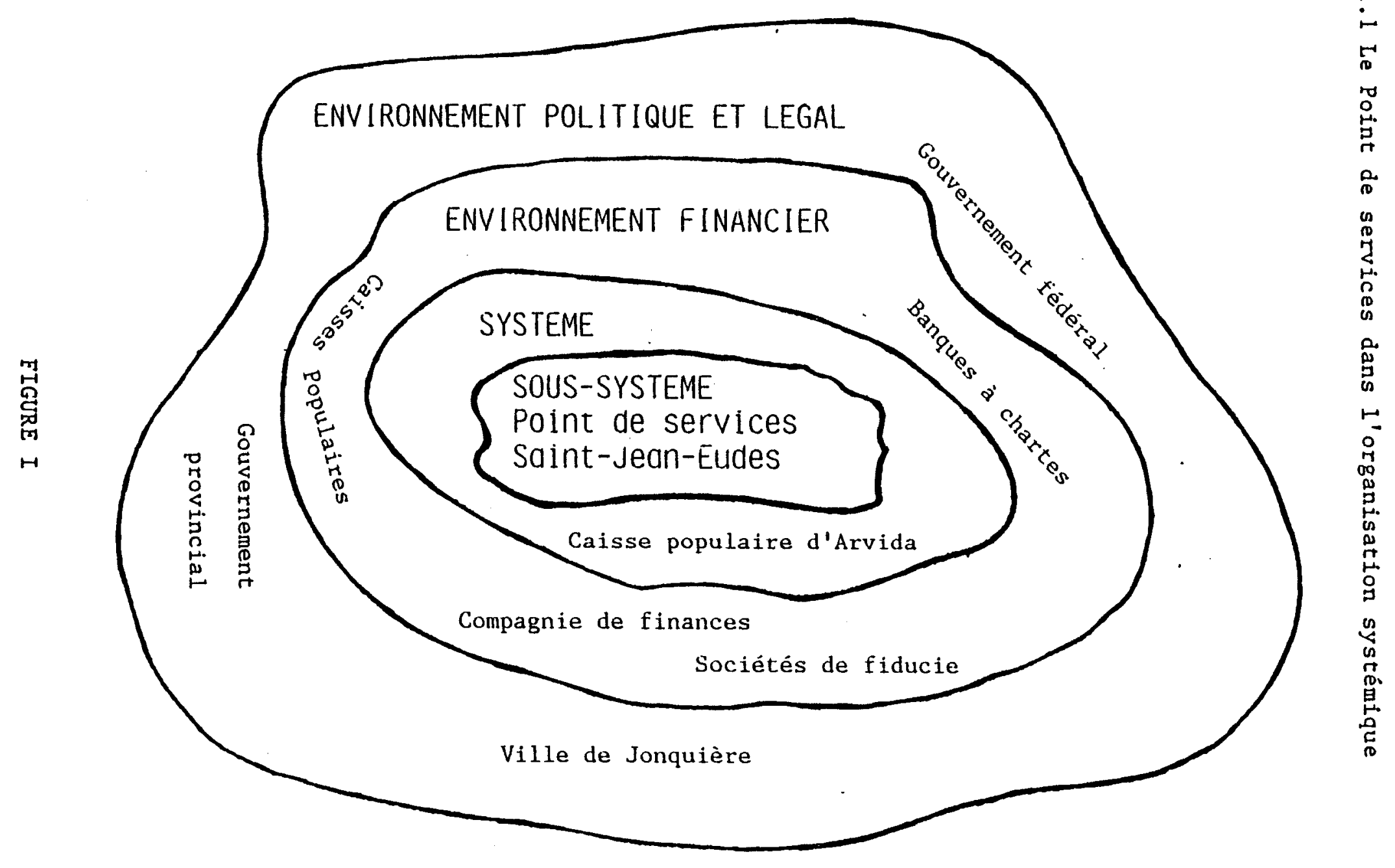




\subsubsection{Environnement socio-géographique}

Le Point de services de la caisse populaire d'Arvida à Saint-Jean-Eudes dessert une clientèle comprise dans la paroisse du même nom. Mais cette population est partagée entre les villes de Jonquière et de Chicoutimi. La plus grosse partie de la clientèle du Point de services se retrouve donc dans les limites de la ville de Jonquière. Et qui plus est, cette population est totalement isolée du reste de la ville par la présence des usines de l'Alcan. Autrefois, il y a près de vingt ans, cette population était implantée dans un territoire érigé en municipalité: celle de Saint-Jean-Eudes.

Un sondage mené à l'été 1985 auprès de la clientèle du Point de services nous a permis d'établir un certain profil-client de ce Point de services. Il faut d'abord noter une présence assez marquée de gens à la retraite, ou vivant de l'aide sociale, ou encore de prestations d'assurance-chômage. Il importe aussi de souligner que, lors des jours où le sondage fut mené, une forte proportion des comptes clients était représentée au comptoir par l'épouse (ou compagne), laquelle venait assez régulièrement à pied. Il existe aussi une bonne quantité de déposants âgés entre 20 et 35 ans. Cette révélation du sondage laisserait entrevoir une possibilité de rajeunissement de la clientèle du Point de services. Si le sondage est exact et précis, près de $50 \%$ des membres de la Caisse à Saint-Jean-Eudes auraient moins de 35 ans. L'annexe V à la fin de l'étude nous fournit plus de renseignements sur le profil-client du Point de services à Saint-Jean-Eudes. 
Au cours des dernières années, il s'est construit par l'arrière de l'édifice abritant le Point de services plusieurs maisons à logements de type HLM. Cette catégorie de gens, clients réels ou potentiels, laisse anticiper la possibilité d'un certain ralentissement de l'activité économique au Point de services. Nous pourrions alors assister à une orientation de l'épargne axée surtout sur l'épargne avec opérations.

Lors de différentes visites dans les locaux du Point de services, nous avons pu observer qu'un très grand nombre de sociétaires se connaissaient très bien. Les jeunes connaissent les plus vieux et vice-versa. Ainsi donc, la caisse devient presque un centre social où tu es certain, lors de ta présence sur les lieux, de rencontrer un parent, un ami, une connaissance. L'endroit sert très souvent de poste de transmission des nouvelles du milieu. Il n'est donc pas rare de voir deux ou plusieurs clients continuer leur conversation pendant un certain temps une fols leurs affaires terminées.

Comme le Point de services est situé sur le boulevard Saguenay conduisant vers Chicoutimi, un autre type de clients s'est greffé à ceux du secteur. En effet, très régulièrement, les caissières doivent donner services à des gens de Chicoutimi et des environs qui s'arrêtent au Point de services sur le retour du travail. La plus grande partie de ces gens travaillent à l'Alcan. Pour justifier leur présence à la Caisse, ils invoquent qu'ils ont quand même accès à leur compte par le système Inter-caisse; enfin, à l'heure où ils passent, ils attendent moins longtemps qu'à leur propre place d'affaires. 


\subsubsection{Environnement financier}

Séparée géographiquement du grand Jonquière par les usines d'Alcan et du centre de Chicoutimi par le domaine des Jésuites et un quartier résidentiel quelque peu industrialisé, la population de Saint-Jean-Eudes se trouve inévitablement coupée de la majorité des services financiers. Ainsi done, le Point de services de la Caisse populaire d'Arvida à Saint-Jean-Eudes constitue la seule institution financière à offrir les services bancaires de dépôts et de retraits dans I'entourage immédiat.

Les services financiers les plus rapprochés de Saint-Jean-Eudes sont situés à Jonquière. Ils sont regroupés au Carré Davis, soit le centre commercial de l'ancienne ville d'Arvida. La. principale place d'affaires de la Caisse populaire d'Arvida se situe dans ce quadrilataire. Elle est entourée des trois banques suivantes: Royale du Canada, de Montréal et Nationale du Canada. La société de fiducie "Le Trust Royal" y offre également les services d'épargne et de crédit, dépôts et retraits; il en est de même pour la caisse d'économie des employés de I'Alcan, affiliée au Mouvement Desjardins. En direction de Chicoutimi, I'institution financière la plus rapprochée est le point de services de 1a Caisse populaire de Chicoutimi sur la côte de Réserve. A Chicoutimi, les concurrentes financières ont leur place d'affaires sur la rue Racine; on $y$ compte au moins six banques, deux caisses populaires et quatre compagnies de finance. 
En raison de cet éloignement, les entreprises financières du Carré Davis à Jonquière représentent donc le principal pôle de concurrence du Point de services. Deux faits importants permettent de soutenir une telle assertion. D'abord le sondage mené auprès des membres de la Caisse, à Saint-Jean-Eudes en juillet et août 1985, révèle que plus de $70 \%$ des répondants travaillent à Jonquière (en $y$ incluant Saint-Jean-Eudes). L'annexe $V$ fournit des renseignements plus précis à ce sujet. Deuxièmement, les sociétaires de Saint-Jean-Eudes qui veulent obtenir des services spécialisés d'épargne et de crédit doivent se rendre au siège social de la Caisse au Carré Davis. La concurrence ne peut donc que se faire à cet endroit de la ville. D'ailleurs le sondage de juillet-août 1985 le précise assez clairement lorsque les répondants confirment, pour plus de 50\%, qu'ils font affaires avec une autre banque et que $59 \%$ de ces derniers font affaires avec une banque sise au Carré Davis.

Il a malheureusement été impossible d'obtenir les statistiques financières des institutions concurrentes. Un fait demeure quand même facilement observable; en effet, lors des jours d'affluence les jeudis et vendredis, les files d'attente sont beaucoup plus longues à la Caisse qu'à n'importe laquelle autre institution financière du secteur. Pourtant on compte autant ou plus de guichets de services à la Caisse. De plus, pour faire face à une croissance assez importante, du nombre de ses sociétaires, la Caisse populaire est à finaliser un projet d'agrandissement de son siège social sur le boulevard Mellon. De 1982 à 
1985, la croissance nette du nombre de membres a été de 488: une augmentation de 5,4\% par rapport à 1982. Le tableau III de 1a page 17 fournit d'autres précisions quant à l'évolution de la clientèle de la Caisse.

Par ailleurs, les services financiers regroupés dans les secteurs Jonquière et Kénogami de la ville de Jonquière de même que ceux localisés dans l'environnement des deux centres commerciaux à Chicoutimi ont une influence plutôt marginale. Soulignons seulement leur existence dans ces secteurs.

Enfin, la Caisse populaire d'Arvida est présentement la seule institution du secteur à offrir les services de dépôts et de retraits par le système du guichet automatique. Les guichets automatiques les plus. rapprochés sont ceux des banques Royale et Nationale de Place CentreVille de Jonquière; ceux de la Banque Nationale et de la Caisse populaire sur la rue Racine en haut de la côte à Chicoutimi; celui de la caisse populaire de Place du Royaume à Chicoutimi. Ce service s'avère donc une raison de plus qui pourrait motiver les gens de Saint-Jean-Eudes à devenir membre de la Caisse populaire d'Arvida.

\subsubsection{Environnement légal et physique}

\subsubsection{Encadrement municipal}

- Pour le Point de services à Saint-Jean-Eudes, la Caisse populaire d'Arvida est liée à Ville de Jonquière par un bail de location d'une durée d'un an. La ville ne veut pas consentir un bail à long ter- 
me, car elle est désireuse de se départir de cet immeuble abritant le Point de services. Le présent bail prend fin au 31 mai 1987.

- En 1985, Ville de Jonquière a procédé à 1'élargissement du Boulevard Saguenay, face à l'édifice de la Caisse. A cause du caractère principal de cet artère routier, un règlement de la circulation interdit le stationnement de véhicules en bordure du chemin. Ces deux situations ont eu pour effet de réduire les aires de stationnement de la Caisse à une quinzaine. De plus, un terre-plein sépare les voies de circulation du boulevard face à la Caisse; d'où obligation pour les sociétaires qui viennent de direction est-ouest (Chicoutimi vers Jonquière) d'aller faire un détour jusqu'aux feux de circulation un peu plus haut.

\subsubsection{Encadrement légal provincial}

- La création et le fonctionnement d'une caisse populaire sont soumis à la loi provinciale sur les caisses d'épargne et de crédit. Il y a quelques années, le ministre des finances du Québec, Monsieur Jacques Parizeau avait promis une refonte de la loi, dans le but de la rendre plus compatible avec la réalité d'aujourd'hui. Les changements de ministres des finances de même que le changement de gouvernement ont fait que cette loi n'a pas encore été modifiée et répond mal aux attentes des caisses populaires.

- Existe aussi la loi de l'assurance-dépôt du Québec qui protège les argents des sociétaires en cas de difficultés majeures comme la situation délicate vécue par les caisses d'entraide économique il y a quelques années. 


\subsubsection{Encadrement légal fédéral}

- Loi canadienne sur les banques à charte

Les caisses populaires ne sont pas soumises à la loi canadienne sur les banques à charte. Ainsi donc, une caisse populaire ne peut transiger directement avec la banque du Canada. Pour contourner la difficulté le Mouvement Desjardins a créé la Caisse centrale Desjardins, laquelle a obtenu une incorporation en vertu de cette $10 i$.

- Association canadienne des paiements

Le mouvement Desjardins est membre de cette association depuis 1983. Cette association permettait à la Caisse centrale Desjardins de recevoir une partie du cent millions de dépôt sans intérêt que le gouvernement fédéral maintenait dans le systéme bancaire. La nouvelle entente de 1986 aura pour effet de rémunérer le système des caisses populaires pour le transit, pour les chèques encaissés et pour les remises de taxes.

\subsubsection{Encadrement de régie interne du Mouvement Desjardins}

- La création d'un point de services

Aucune réglementation de régie interne ne délimite un cadre de procédure quant à la création et au maintien d'un réseau de points de services par une caisse populaire. 
- Les réserves:

1) En vertu des réglements de la Fédération, la Caisse est obligée de maintenir à la Fédération un fonds de liquidité qui se situe entre 8 et $12 \%$ de 1 a valeur de l'ensemble des dépôts.

2) La Caisse doit aussi verser au fonds d'investissement une partie des dépôts reçus.

3) Enfin la Caisse maintient un fonds de dépôt à terme lorsqu'il y a trop d'argent dans le fonds de liquidité.

- Convention collective

Depuis environ quatre ans les relations de travail entre patrons et employés sont chapeautées par une convention collective signée entre les parties. Celle-ci est venue à échéance à la fin du mois d'août 1986. On est donc en cours de négociation pour le renouvellement de la convention collective de travail des employés.

Tous les aspects de l'environnement qu'on vient de décrire ont mis en exergue plusieurs éléments qui pourraient influencer le développement et la croissance du Point de services. Soulignons entre autres le caractère d'exclusivité qui émane de la description de l'environnement socio-géographique et de l'environnement financier. Ce caractère devrait, à notre avis, inciter les preneurs de décisions à en tenir compte lorsque sera venu le temps d'envisager quelque modification que ce soit dans l'organisation du Point de services. S'il en était autrement, 
il apparaftrait illusoire de vouloir procéder à une analyse plus approfondie de cette entreprise de services financiers. Pour cette raison, l'étude des structures et la description des tâches qui suivent se doivent d'être entreprise dans I'optique de services exclusifs à fournir sur place à la collectivité de Saint-Jean-Eudes.

\subsection{DESCRIPTION DES STRUCTURES}

Plusieurs auteurs et/ou administrateurs prétendent, à juste titre probablement, qu'une entreprise, qui a bien établi ses structures organisationnelles et bien défini les tâches à exécuter, détient la clé de l'efficacité, voire de la rentabilité de son organisation. La description des structures, avec lesquelles le Point de services peut offrir les services financiers nécessaires à la population du milieu, devrait permettre de vérifier le bon fonctionnement de celui-ci et d'en déceler les forces et faiblesses.

La figure II, présentée à la section 2.2 .1 qui suit, reproduit I'organigramme de 1a structure administrative de la Caisse populaire d'Arvida. Mais un tel cadre hiérarchique n'acquiert une valeur et sa nécessité que s'il est confirmé et appuyé par une description succincte des tâches à exécuter.

Et pour mieux saisir son importance, la description des tâches est accompagnée d'une présentation sommaire des politiques opérationnelles et d'un plan à l'échelle de l'aménagement physique des lieux. 


\subsubsection{Organigramme de 1 'institution}

Comme il n'y a pas de personnel cadre, responsable, sur place, de l'administration totale du Point de services, nous avons cru bon de présenter I'organigramme de la Caisse populaire d'Arvida. A cet organigramme, nous avons ajouté un poste spécial pour bien illustrer, dans la structure administrative, la dépendance du personnel caisster du Point de services. Il s'agit du poste marqué d'un astérisque. 
ORCANIGRAME

CAISSE POPULAIRE D'ARVIDA

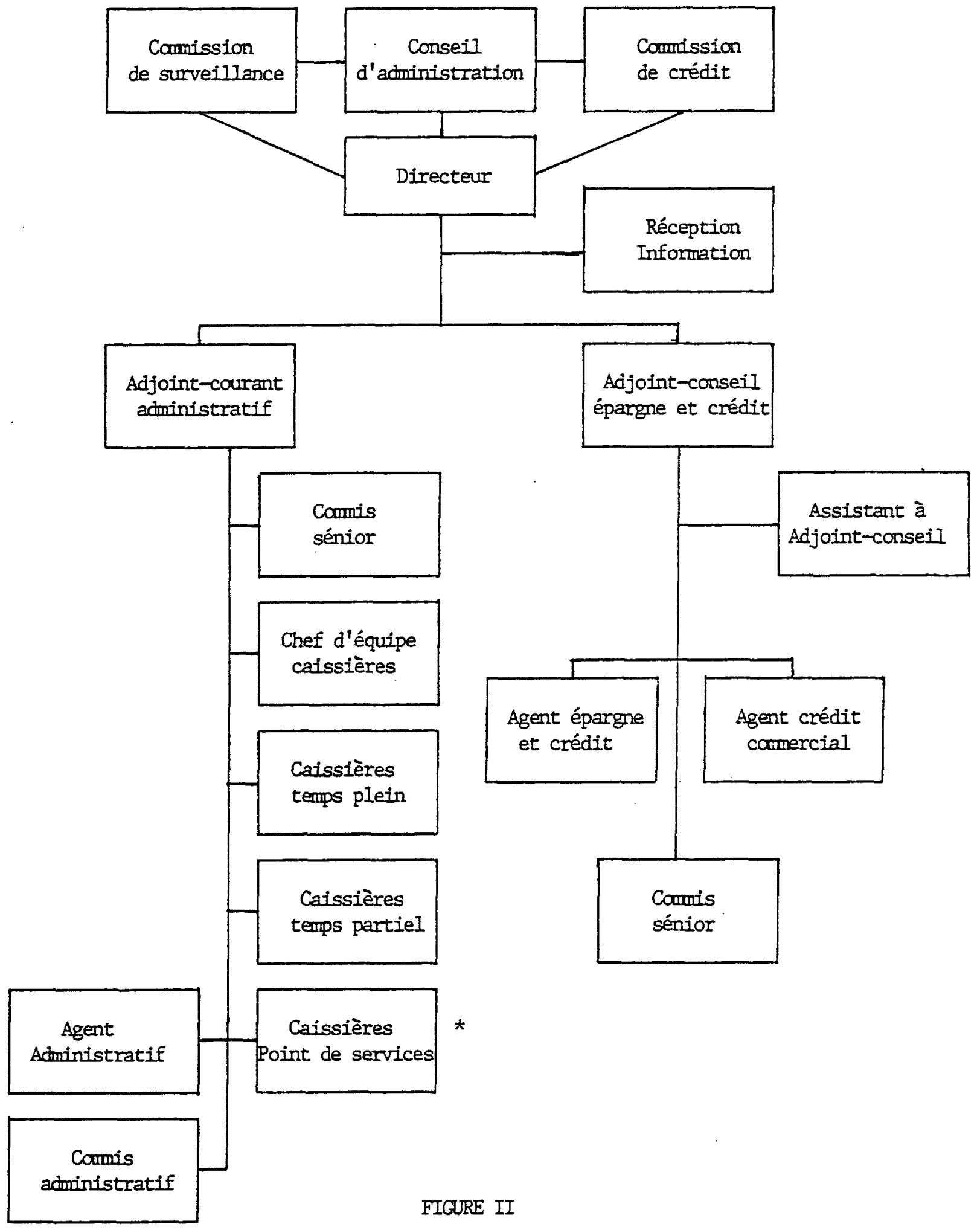




\subsubsection{Description des tâches}

Pour les besoins de notre recherche action, nous avons retenu seulement les tâches qui avaient un lien direct avec les sociétaires de Saint-Jean-Eudes et/ou les services à leur offrir. Ce sont en l'occurence, les trois tâches de cadres avec quelques ramifications pour l'adjoint conseil, la tâche de caissière et celle de chef d'équipe.

\subsubsection{Le directeur}

Dans une caisse populaire, le directeur est le mandataire du conseil d'administration qui procède à son (ré) engagement à chaque année. Ses principales responsabilités se résument ainsi:

- kSous I'autorité du conseil d'administration, le directeur planifie, dirige et contrôle l'ensemble des activités en vue d'atteindre les objectifs de la Caisse.

- Il associe les membres et les dirigeants à la gestion de la Caisse.

- Il répond, de concert avec les dirigeants, aux besoins de la collectivité.

- Il établit, de concert avec les dirigeants, les liens entre la Caisse et d'autres secteurs coopératifs." ${ }^{1}$

1- Le Manuel SOC, Fédération de Québec des caisses populaires Desjardins, Section G0-310-6, p.1 
Pour accomplir sa tâche, le directeur reçoit l'aide de deux adjoints qu'on nomme ainsi:

- Adjoint courant et administratif.

- Adjoint conseil épargne et crédit.

Très rarement, on devrait dire presque jamais, le directeur doit se rendre rencontrer les membres ou les employés au point de services de Saint-Jean-Eudes.

Le directeur actuel de la Caisse populaire d'Arvida est en poste depuis un peu plus de six ans. Il détient un diplôme d'étude collégiale en finance. Présentement, il ne paraît pas intéressé à rechercher un baccalauréat en cette discipline. Cela ne 1'empêche toutefois pas de maintenir sa compétence administrative à point parce que, régulièrement, il s'inscrit à des cours universitaires sur des sujets reliés à son travail. De plus, il rate rarement l'occasion de suivre les séances de formation organisées par la Fédération des caisses populaires Desjardins du Saguenay-Lac-Saint-Jean. Ces cours traitent surtout de sujets se rapportant au fonctionnement des coopératives d'épargne et de crédit.

\subsubsection{Le directeur-adjoint aux services courants et administra- tifs.}

A la Caisse populaire d'Arvida le titulaire de ce poste remplit une double fonction. Il est responsable des services courants (services du personnel) en plus de coordonner les services administratifs. 
Comme adjoint aux services courants, ses responsabilités sont les suivantes: "Sous 1'autorité du directeur de la Caisse, le directeuradjoint aux services courants planifie, organise, dirige et contrôle les activités relatives à la vente de services de guichet, de comptoir d'appoint et autres services financiers." ${ }^{2}$

Comme adjoint administratif, ses responsabilités se résument ainsi: "Sous l'autorité du directeur de la Caisse, le directeur-adjoint aux services administratifs planifie, organise, dirige et contrôle les activités de son secteur, consistant à fournir un support à l'ensemble des secteurs d'activités relativement à la gestion des ressources humaines, financières et techniques. $\|^{3}$

En termes plus simples on pourrait dire qu'il dirige les employés de la Caisse, en organise le travail et voit à l'embauche si nécessaire. Il est responsable de I'équipement. Face aux sociétaires et des autres, il informe sur les services offerts et en organise la publicité.

L'actuel adjoint courant et administratif est le premier titulaire de ce poste, créé il y a un peu plus de deux ans. Avant d'occuper ce poste, ce dernier travaillait à la succursale de la Banque

2- Manuel SOC. Fédération de Québec des caisse populaires Desjardins, Sept 1979, Section GO-330-1. p.1

3- Ibidem. Section GO-350-1. P.I 
Nationale du Canada sur la rue Racine à Chicoutimi. Il détient un diplôme d'étude secondaire du cours commercial. Tout récemment, il obtenait un certificat en administration de l'Université du Québec à Chicoutimi.

\subsubsection{Le directeur-adjoint aux services conseil}

Le deuxième adjoint au directeur de la Caisse s'occupe des services spécialisés d'épargne et de crédit. Le cahier de la description des postes définit de cette façon ses responsabilités: "Sous I'autorité du directeur de la Caisse, le directeur adjoint aux services conseil planifie organise, dirige et contrôle les activités relatives à la vente de services intégrés d'épargne et de crédit et autres services financiers." 4

C'est donc aux services conseil que les sociétaires vont s'adresser pour obtenir des informations sur les différentes façons de placer leur argent et procéder à ces placements. Ce sont également les mêmes services que les membres utilisent pour négocier les différents prêts disponibles.

Le directeur adjoint aux services conseil doit faire rapports et recommandations au directeur de la Caisse.

4- Ibidem, Section G0-340-1, p.1 
Depuis la fin de juillet 1986, les services conseil de la Caisse populaire d'Arvida sont sous l'autorité d'un nouvel adjoint-conseil. Avant d'entrer en fonction à la Caisse, ce dernier était directeur (gérant) dans une succursale d'Arvida d'une banque concurrente. Il succède à l'ancien directeur adjoint, lequel devient assistant au directeur adjoint des services conseil (nouveau poste). Ce dernier occupait depuis plusieurs années le poste d'adjoint-conseil.

La direction des services conseil est régulièrement en contact avec les sociétaires du Point de services. Mais en aucun temps, les services spécialisés d'épargne et de crédit ne sont donnés à SaintJean-Eudes. Les sociétaires de Saint-Jean-Eudes doivent donc se rendre au siège social de la Caisse pour bénéficier de ces services.

La direction des services conseil est également appuyée par deux agents. A l'épargne et au crédit personnel, les sociétaires rencontre un agent spécialement formé. Quant aux besoins de crédit commercial, ils sont comblés par un agent spécialisé en ce domaine.

\subsubsection{La chef d'équipe}

Etant sous l'autorité du directeur ajoint aux services courants et administratifs, la chef d'équipe a comme principale fonction de préparer l'horaire hebdomadaire des caissières. C'est donc elle qui détermine 1 'horaire de travail des caissières du Point de services et qui seront ces caissières. De plus elle fournit une aide technique aux dites-employées ce qui a pour effet de l'amener de temps à autres au Point de services. 


\subsubsection{Les caissières}

$\mathrm{Au}$ Point de services, les caissières effectuent les opérations de guichet, soit les dépôts, les retraits et les perceptions diverses. Elles sont donc les employées les plus souvent en contact avec les sociétaires de Saint-Jean-Eudes. Trois caissières effectuent du travail de guichet à Saint-Jean-Eudes. Une travaille à temps complet (35 heures par semaine). Une deuxième se rend au comptoir pour 27 heures. Et depuis le début de l'été 1986, une troisième caissière vient travailler environ 6 heures par semaine. Elle remplace les deux autres sur I'heure du dîner les jours d'affluence. Trois caissières sont particulièrement utilisées pour donner le service à Saint-Jean-Eudes.

Celle qui était la caissière attitrée à temps complet depuis 9 ans vient d'être mutée au siège social pour des raisons professionnelles et techniques.

\subsubsection{Les politiques opérationnelles et règlements}

Le Point de services de Saint-Jean-Eudes ouvre ses portes aux sociétaires du lundi au vendredi inclusivement. Les lundis, mardis et mercredis, les services sont offerts de 10 à 15 heures. Quant aux jeudis et vendredis, les services sont continus de 10 à 18 heures. Avec un terminal donnant accès à l'ordinateur central du Mouvement Desjardins, les membres ont accès à tous les services de dépôts et retraits au guichet, y compris I'inter-caisse. Tout comme dans les autres banques ou caisses, le personnel caissier est autorisé à percevoir les comptes payables en ces endroits. 


\subsubsection{Travail des caissières}

Seule la caissière à temps complet doit se rendre au siège social dès 9 heures le matin. Là, elle y reçoit les directives et renseignements du directeur-adjoint aux services courants et administratifs. Elle prépare le matériel dont elle aura besoin et doit assurer son transport au Point de services pour $9 \mathrm{~h} 45$ heures. Lorsqu'une caissière est affectée à du temps partiel au Point de services, elle se rend directement à son lieu de travail. Une fois la journée terminée et la balance des caisses établie et vérifiée, encore une fois, seulement la caissière à temps complet se rend au siège social pour donner son rapport et classer les effets de la journée.

\subsubsection{Rôle de I'adjoint courant et administratif}

Une fois la semaine, il se rend au Point de services pour faire une vérification du trésor. Il peut à l'occasion se rendre à Saint-JeanEudes pour une vérification surprise de caisse, ou régler des urgences comme le manque de numéraire par exemple.

\subsubsection{Transport du numéraire}

Une fois par semaine, habituellement le mardi matin, la société Sécur, organisme du mouvement Desjardins, assure le Point de services d'un approvisionnement suffisant en numéraire. S'il arrivait un manque à ce niveau, il revient au directeur-adjoint aux services courants et administratifs ou à la chef d'équipe d'assurer l'approvisionnement à la place de la société Sécur. Par mesure de sécurité, la caissière refuse d'accomplir cette opération. 
Tout cet exercice de description de la structure de fonctionnement du Point de services qui vient d'être faite vise à fournir une gamme variée et précise de renseignements permettant aux décideurs de dégager des situations problématiques présentes ou à venir. Cet exercice ne saurait être complet sans un portrait assez détaillé de l'aménagement physique des lieux.

\subsubsection{Aménagement physique des lieux}

Les deux figures suivantes présentent une description à l'échelle de l'intérieur des locaux du Point de services et de I'aménagement des aires de stationnement. 
AMENAGEMENT INTERIEUR DU POINT DE SERVICES

Echelle: $1 / 4^{* \prime}=2$ pieds $\quad$ Ramené à $75 \%$

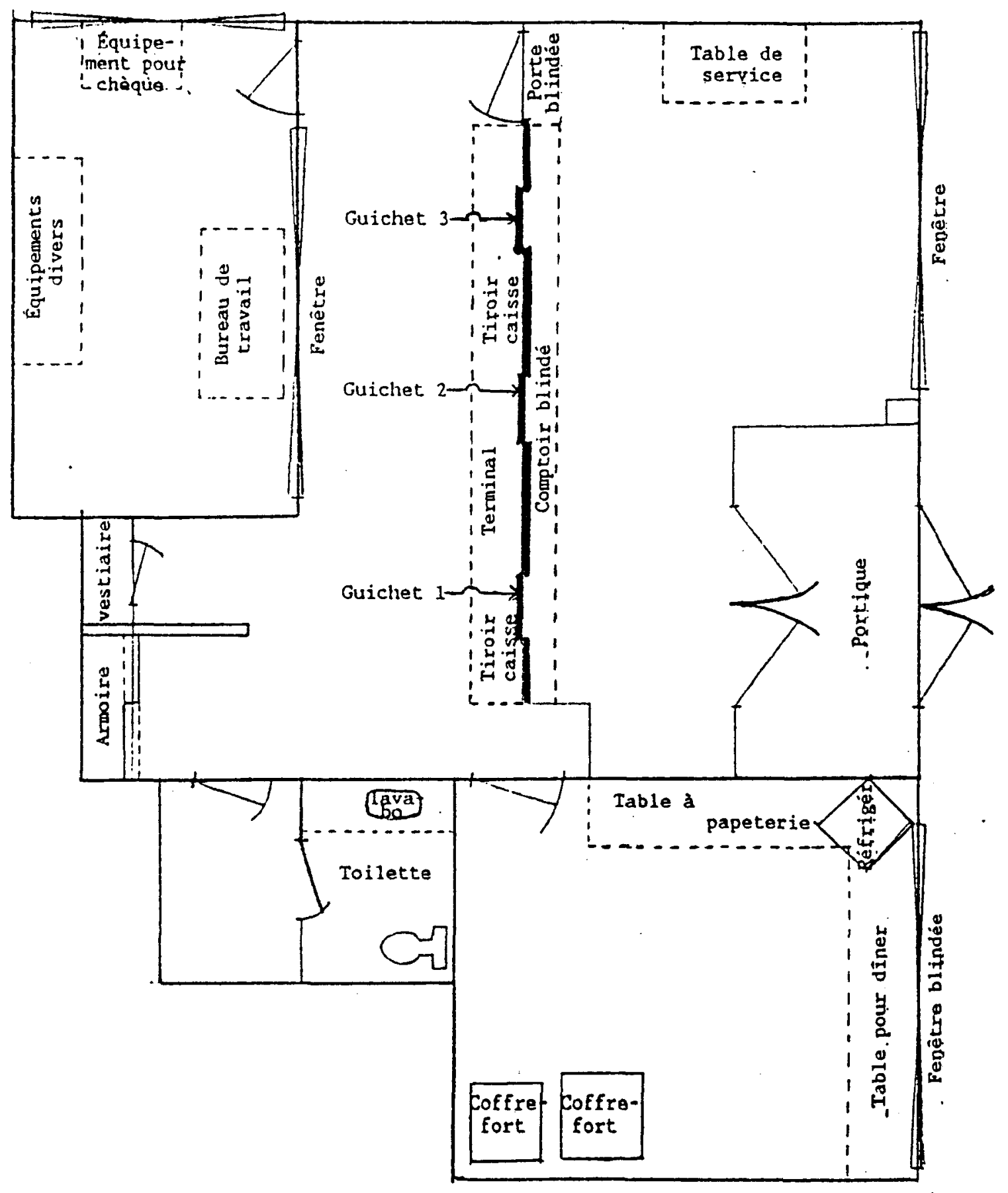

FIGURE III 
Echelle: $1 / 4^{\prime \prime}=4$ pieds

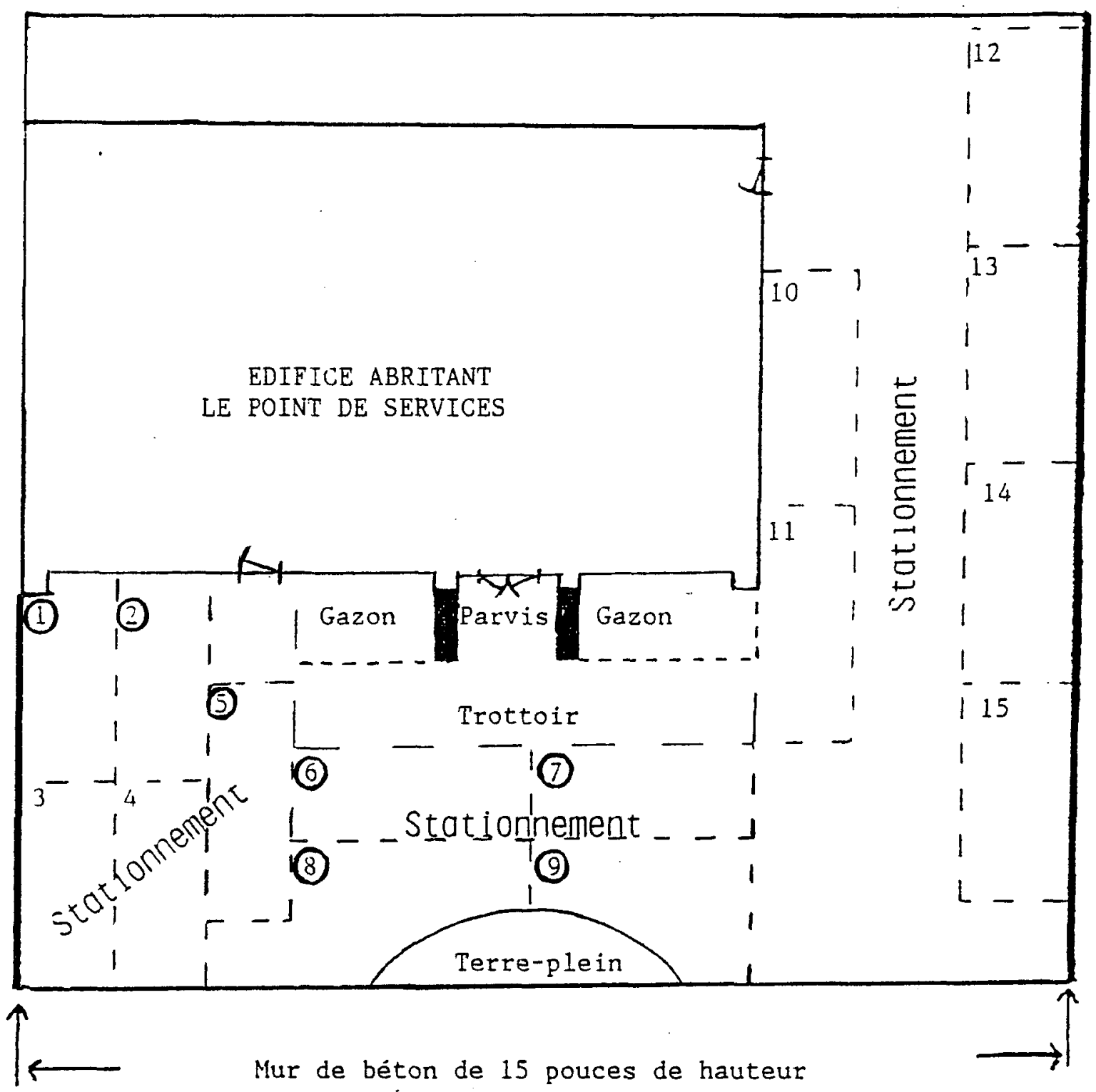


En résumé, l'analyse des structures permet de dégager les faits suivants. Peu de personnes sont impliquées dans l'offre directe de services aux sociétaires au Point de services. Le plán de I'aménagement physique des locaux révèle que les deux caissières de service occupent un espace très grand par rapport à celui alloué aux membres pour I'attente aux guichets. Enfin, les aires de stationnements semblent en nombre suffisant, mais d'un encombrement facile aux endroits marqués d'un cercle noir.

\subsection{DESCRIPTION DU PROCESSUS}

Après l'analyse des structures, la description du processus vient compléter la présentation du système organisationnel desservant les sociétaires de Saint-Jean-Eudes. Le tracé schématique des opérations financières effectuées au Point de services est illustré par les figures $V$ et VI. La figure VII expliquera $1^{\prime}$ arrivée du numéraire au Point de services.

2.3.1 Liste des opérations effectuées

- Recevoir les directives

- Préparer les effets nécessaires

- Se trasporter au Point de services

- Ouvrir le coffre-fort

- Préparer la caisse

- Accueillir les clients 
- Informer les clients

- Transférer au siège social

- Préparer (remplir) les effets pour les transactions

- Recevoir le numéraire

- Percevoir des comptes

- Enregistrer les différentes transactions

- Remettre le change ou l'argent et/ou le livret

- Vérifier les livrets (par le sociétaire)

- Effectuer des contrôles (vérifications)

- Balancer sa caisse

- Placer sa caisse au coffre-fort

- assurer son retour au siège social

- Déposer et classer les effets reçus 
2.3.2 Ordonnancement des opérations

SCHEMA DES OPÉRATIONS

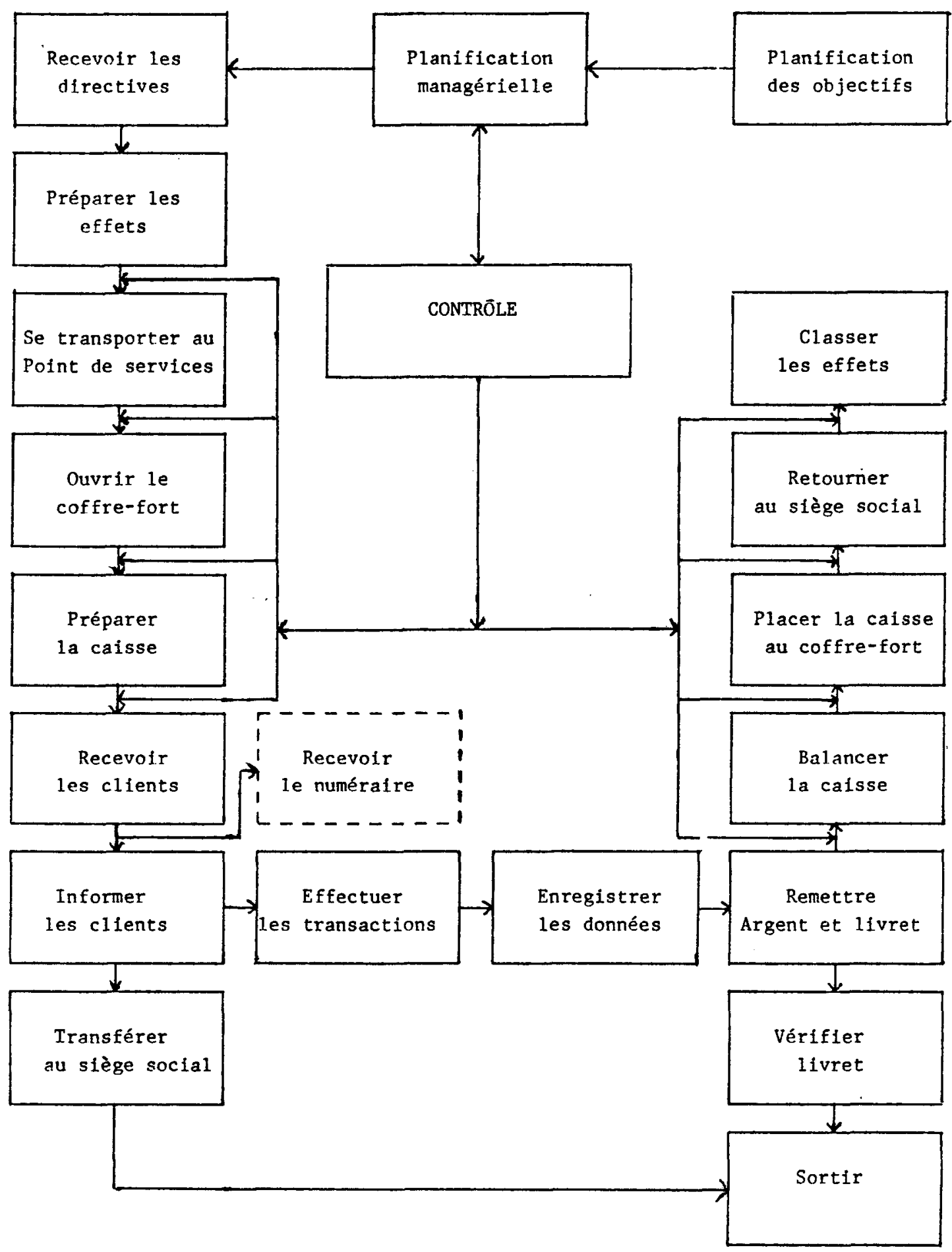

FIGURE V 


\subsubsection{Opérations nécessaires pour effectuer les transactions}

Lorsqu'on se présente au Point de services, on peut effectuer les transactions suivantes. Parfois on fait un dépôt; une autre fois on procêdè à un retrait; un autre moment le sociétaire demandera d'opérer un virement de fonds; ou tout simplement voudra-t-il payer son téléphone, son électricité, ses taxes... Très souvent le membre effectuera plusieurs de ses activités au cours de la même transaction. La figure VI suivante illustre la procédure à suivre au cours d'une transaction.

\section{PROCEDURES D'UNE TRANSACTION}

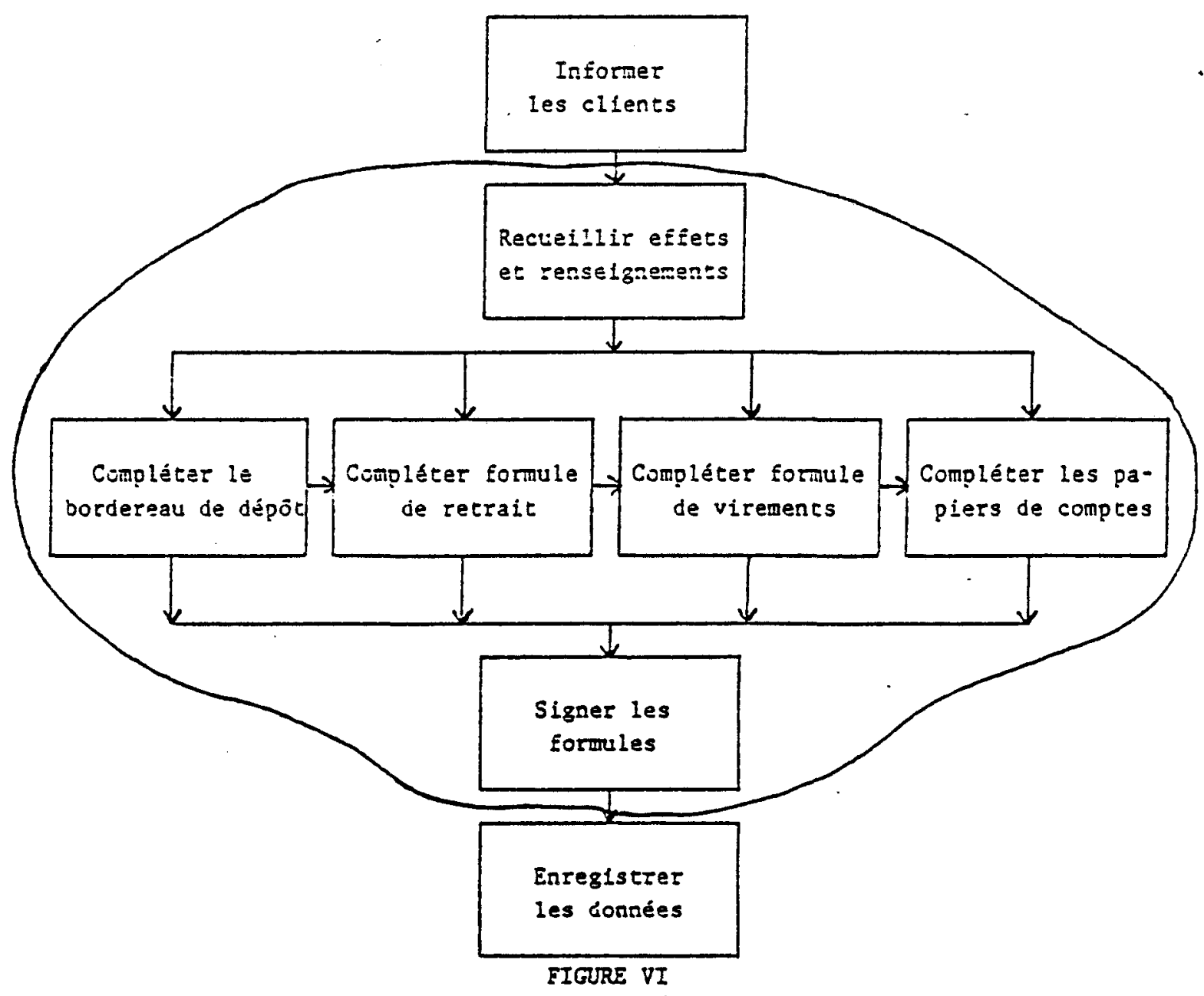




\subsubsection{Opérations relatives au transport du numéraire}

Habituellement le mardi matin, la Société Sécur, se rend au Point de services, pour assurer le transport du numéraire. Qu'il y ait clients ou non, la caissière se doit de donner priorité à l'officier de Sécur et effectuer les opérations requises jusqu'au départ de cet officier. La figure suivante illustre les opérations à faire.

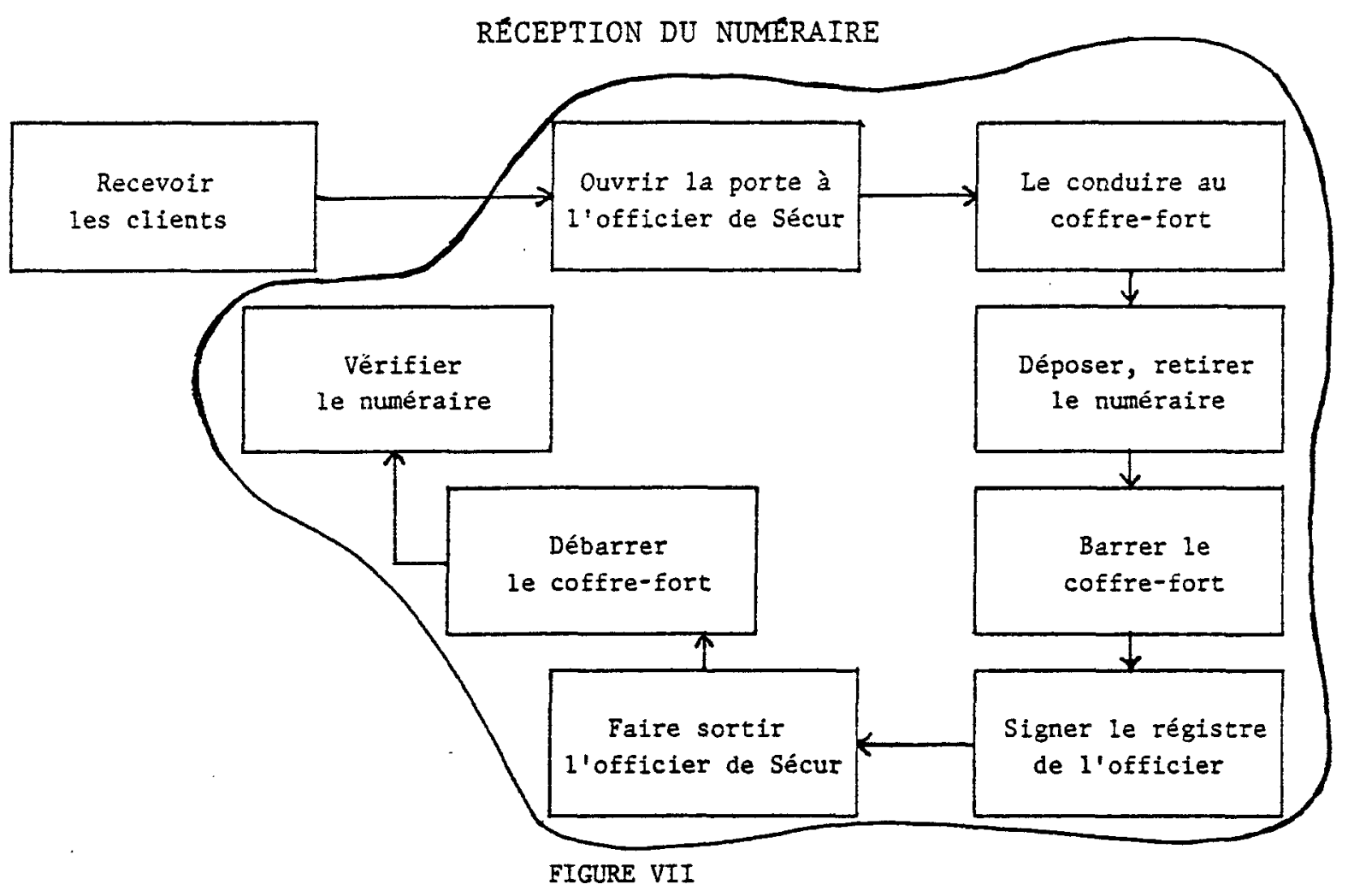

Lorsqu'il y a réception de numéraire, le bon de commande est placé dans le sac apporté par la Soclété Sécur. Comme la caissière doit barrer le coffreffort, par mesure de sécurité, après le dépôt de l'argent, le contrôle de la monnaie nouvellement arrivée s'effectuera après le départ des officiers de Sécur. Il revient à la caissière d'effectuer cette vérification, laquelle se fera en l'absence de clients. 
Avec la description du processus se termine la présentation technique du Point de services. Cette présentation s'est effectuée en trois volets. Nous avons d'abord situé le Point de services dans l'environnement qui lui était propre. Par la suite nous avons décrit la structure organisationnelle mise en place pour offrir les services financiers aux sociétaires. Enfin nous avons rattaché à cette structure la façon dont les services étaient offerts en traçant le portrait du processus opérationnel lui-même.

La section suivante de l'élaboration de la problématique délimitera d'une certaine façon la philosophie qui sous-tend le maintien d'un point de services de la Caisse populaire d'Arvida à Saint-JeanEudes. Pour construire cette pensée, cette opinion, nous allons procéder à la description du climat organisationnel en relatant les points de vue du directeur de la Caisse et du directeur-adjoint aux services courants et administratifs. Certains avis des caissières seront également colligés.

\subsection{LA DESCRIPTION DU CLIMAT ORGANISATIONNEL}

Depuis la création de son poste en 1984, le directeur-adjoint aux services courants et administratifs de la Caisse hérita de I'administration et du fonctionnement du Point de services. La hiéarchie administrative illustrée par l'organigramme de l'institution à la figure II de la page 38 révèle cette orientation administrative. 
2.4.1 Le point de vue du directeur-adjoint aux services courants et administrafifs

Premier titulaire du poste qu'il occupe, 1'actuel directeuradjoint aux services courants et administratifs est venu offrir à la Caisse sa solide expérience du milieu bancaire. En effet, après des débuts comme commis dans une succursale de la Banque Nationale à Chicoutimi, son dynamisme et sa compétence l'ont vite amené au siège social de la banque à Montréal. A cet endroit, il occupa successivement les fonctions de conseiller en ressources humaines et d'agent de personnel. De retour à la même succursale à Chicoutimi en 1981 comme directeur-adjoint à l'administration, la Caisse requiert ses services trois ans plus tard pour un poste similaire.

Quelque temps après son entrée en fonction, l'adjoint courant et administratif s'est interrogé sur le Point de services. Habitué qu'il était à penser la rentabilité d'une entreprise en fonction des profits à réaliser, il aurait pris la décision de fermer le Point de services parce qu'il n'était pas assuré de sa profitabilité; d'autant plus qu'il ne rendait qu'un nombre limité de services aux sociétaires. En raison de l'objectif de service à la collectivité, lequel doit guider les activités d'une coopérative d'épargne et de crédit, il a pris conscience qu'il eût été une erreur d'arrêter les opérations du Point de services.

Pour expliquer son raisonnement, il affirmait que, si la Caisse fermait son Point de services, ce dernier ne serait pas remplacé par une succursale d'une banque concurrente. De plus, il ajoutait que I'adminis- 
tration de la Caisse serait très mal vue de la population du secteur si elle prenait une telle décision. Il s'en suivrait certainement une fuite de capitaux.

Dans 1'exercice de ses fonctions, I'adjoint courant et administratif doit se rendre hebdomadairement au Point de services pour procéder à la vérification du solde du trésor des caissières et le contenu de l'encaisse. Parfois il vient effectuer un décompte surprise du contenu de la caisse de chacune des employées. Les horaires de travail établis par la chef d'équipe sont généralement respecté par I'adjoint courant et administratif. Cette façon de procéder ne semble pas affecter le travail des caissières et répond aux besoins de 1 'adjoint.

Lors de ses visites sur les lieux du Point de services, le directeur-adjoint aux services courants et administratifs en profite pour observer le déroulement des activités. Suite à ces observations, il a constaté que l'aménagement physique des lieux nuisait au bon fonctionnement du Point de services. Sa remarque la plus importante est à l'effet que le système actuel des files d'attente gène considérablement I'accès des clients lors des jours d'affluence.

Toujours selon I'adjoint courant et administratif, la présence du mur anti-balles, installé pour éliminer les vols quasi hebdomadaires, cause également des obstacles au bon fonctionnement du Point de services. Dans sa construction actuelle, il empêche l'installation des services spécialisés d'épargne et de crédit. En outre, la caissière et son client 
doivent se parler assez fort pour être un mesure de se comprendre; ce qui réduit presqu'à néant le caractère confidentiel ou personnel de certaines transactions.

Dans sa structure physique actuelle, le Point de services ne peut guère progresser au delà de ce qu'il est. Tant qu'il n'aura pas été réaménagé ou relocalisé, le Point de services verra la qualité de ses services stagner, sa croissance financière se stabiliser au niveau actuel ou décroître par rapport aux autres milieux financiers. C'est en somme la conclusion de la pensée du directeur adjoint aux services courants et administratifs.

\subsubsection{Le Point de vue du directeur de la Caisse}

Pour l'actuel directeur de la Caisse, le système des caisses populaires et d'économie Desjardins n'a plus beaucoup de secrets. II compte en fait quatorze années d'expérience au sein du mouvement Desjardins dont les six dernières comme directeur de la Caisse populaire d'Arvida. Tous ses antécédents font que la grande force du directeur réside dans sa capacité d'analyse financière. C'est sans doute pour cette raison que sa priorité comme directeur de la Caisse consiste à atteindre avant la fin de la présente année financière, en novembre 1986, I'objectif suivant: "Générer suffisamment de trop-perçu pour atteindre la couverture légale soit $3,5 \%$ du passif-dépôt. ${ }^{5}$

5- Extrait du cahier des objectifs déterminés par les dirigeants de la Caisse populaire d'Arvida le 26 octobre 1986. 
Tout en étant préoccupé par le dossier du Point de services, ce dernier n'apparaît pas comme une priorité immédiate pour le directeur. Cependant, lorsqu'il parle du devenir de ce comptoir de services, il prétend qu'il serait possible d'en augmenter le chiffre d'affaires actuel qui tourne autour de 2,5 millions de dollars. Toujours selon le directeur, ce qui fait défaut à Saint-Jean-Eudes c'est 1'absence de services spécialisés te1: REER, COFFRETS-DE-SURETË, DËPOTTS-Ã-TERME, PRETS..., directement sur place. La disposition physique des lieux et le mur anti-balles empêchent 1'organisation de tels services. Dans 1a situation actuelle, le, caractère confidentiel de ce genre de transactions ne saurait être entièrement conservé si on offrait ces services spécialisés.

Selon le directeur, il y aurait quand même possibilité d'améliorer la qualité du service à la clientèle par la modification des points suivants:

- A I'aide d'un système de câbles, réunir en une seule les deux files d'attentes, facilitant ainsi l'accès au local à un plus grand nombre.

- Accélérer le service aux sociétaires en ajoutant une caissière pour remplacer les deux autres sur l'heure du dîner.

Le directeur affirme enfin qu'il n'est pas question de fermer le Point de services. Car en utilisant le temps de $13 / 4$ caissières, on réussit à drainer près de un million et demi de dollars d'épargne si on 
exclut les dépôts à terme qui se font au Siège social. Il a également porté à notre attention. que le Point de services était la seule institution financière présente dans le milieu. Fermer le Point de services serait alors manquer à la mission des caisses populaires telle que définie par les membres du conseil d'administration de la Confédération des caisses populaires et d'économie Desjardins du Québec soit: "La capacité de la caisse de pouvoir offrir des services d'épargne et de crédit de qualité, compétitifs, rentables, articulés les uns par rapport aux autres et reliés à ses objectifs de promotion socioéconomique de ses membres et de la collectivité dans laquelle elle opère." 6

\subsubsection{Le point de vue des caissières}

Les employés de la Caisse étant régis par une convention collective, la chef d'équipe doit donc tenir compte du critère d'ancienneté quand vient le temps de confectionner les horaires de travail des caissières. Sauf pour quelques besoins spécifiques du directeur adjoint aux services courants et administratifs, la chef d'équipe place presque toujours les mêmes caissières au Point de services. On déplore cependant de leur part le fait que, même si elles ont à accomplir une tâche complète de 35 heures, elles soient encore considérées comme des employées à temps partiel.

6- Le Manuel SOC, Fédération de Québec des Caisses populaires Desjardins, sept 1979, Section G0-100-1, p.1 
Elles sont en contact direct avec les sociétaires du milieu et c'est souvent vers elles que les membres acheminent leurs plaintes qui portent principalement sur le stationnement, les services spécialisés ou encore les files d'attente.

Tout en se déclarant heureuses au Point de services, elles souhaiteraient une certaine amélioration de la propreté des locaux. Elles s'interrogent sur le fait qu'elles soient obligées d'accomplir des tâches qui normalement seraient dévolues à une commis sénior ou à la chef d'équipe. Elles estiment qu'il faudrait améliorer le système de communication audio placé de chaque côté du mur anti-balles. Évidemment elles aiment ce mur anti-balles car 11 leur enlève la crainte de vols à main-armée. Elles refuseraient probablement de travailler si cette protection était absente.

En somme, elles apprécient toute l'autonomie qui leur est procurée par cet éloignement du siège social de la Caisse.

\subsection{ANALYSE DES INDICATEURS}

Dans l'élaboration d'une problématique, l'analyse des indicateurs apparaît comme une des activités les plus importantes. En effet, losque nous procédons à cette phase de travail, nous scrutons sous plusieurs aspects les quatre fonctions essentielles de toute entreprise: Les finances, la gestion des ressources humaines, la gestion de la production et les services de marketing et vente. 
Le but de cet exercice est d'en arriver à l'identification des éléments pouvant engendrer des situations problématiques à 1'intérieur de 1'entreprise. L'ordre de présentation des indicateurs ne signifie pas I'importance de 1 'un par rapport aux autres. Dans la présente rechercheaction, nous débutons par les indicateurs financiers pour terminer par ceux de productivité.

\subsubsection{Les indicateurs financiers}

Lors des réunions mensuelles du conseil d'administration d'une caisse populaire (du moins celle d'Arvida), les administrateurs sont appelés à vérifier la situation financière de leur caisse par la présentation d'un rapport expliqué par le directeur. Ce qui retient l'attention et ce sur quoi va s'appuyer le directeur pour démontrer la saine administration qu'il fait, ce sont les deux facteurs de rentabilités suivants: la marge brute et la situation du trop-perçu net.

Le Point de services ne produisant pas ses propres états financiers, nous avons dû construire, avec l'aide du directeur de la Caisse, sa marge brute et sa situation du trop-perçu net à partir des rapports financiers de la Caisse à la mi- juillet 1986. Toujours en utilisant les mêmes états financiers, nous avons pu établir un bilan pro-forma, portrait du Point de services au 18 juillet 1986. Enfin, grâce à des statistiques financières fournies par la Caisse, grâce à des données statistiques de François Berger de la Fédération des caisses populaires du Centre du Québec, nous avons comparé la croissance du Point 
de services à celle du siège social de la Caisse et à celle d'autres institutions de même type. Les quelques pages qui suivent dévoilent l'état de la santé financière du Point de services.

\subsubsection{La marge brute}

La marge brute est cet instrument de mesure financière qui permet à la caisse de calculer quel est son revenu brut moyen par $100 \$$ de passif-dépôt. Pour y arriver on fait d'abord la sommation de tous les revenus d'intérêt générés par les différentes sortes de prêts. A ceux-ci, on ajoute les revenus d'intérêt réalisés grâce aux placements faits par la caisse. Au Point de services, ces revenus totalisent 288 219 \$, soit 11,83 \$ par 100 \$ de passif-dépôt.

De ces revenus, il faut soustraire les dépenses d'intérêt engendrées par les quatre différentes formes de dépôts. Lors de notre vérification au 18 juillet 1986, ces dépenses représentaient pour les 12 derniers mois une somme de 147423 \$. Le passif-dépôt pour la même période était de 2436299 \$. Donc les dépenses d'intérêt sont de 1 'ordre de 6,05 par 100 \$ de passif-dépôt.

Ainsi, si on effectue la différence entre les revenus d'intérêt et les dépenses d'intérêt, on obtient une marge brute de 5,78 \$ par $100 \$$ de passif-dépôt. Le tableau $\mathrm{V}$ qui suit donne les détails de ce calcul de la marge brute. 
TABLEAU $V$

LA MARGE BRUTE

POINT DE SERVICES

31 JUILLET 1986

\begin{tabular}{|c|c|c|c|c|}
\hline Identification & Valeurs & Taux & Revenus/dépenses & $/ 100$ P.d. \\
\hline \multicolumn{5}{|c|}{ Revenus d'intérēt } \\
\hline Fonds de Iiquidité & 270632 & 8,25 & 22327 & 0,9 \\
\hline Fonds de dépôt à terme & 20000 & 9,00 & 1800 & 0,07 \\
\hline Fonds d'investissement & 43853 & 3,50 & 1535 & 0,06 \\
\hline Prêts R/D & 923565 & 12,7 & 117293 & 4,8 \\
\hline Prêts hypothécaires & 1274244 & 11,4 & 145264 & 6,00 \\
\hline Total (a) & 2532294 & 11,38 & 288219 & 11,83 \\
\hline \multicolumn{5}{|c|}{ Dépenses d'intérēt } \\
\hline Capital social & 19698 & 5,9 & 1162 & 0,05 \\
\hline EOP & 642068 & 0,5 & 3210 & 0,13 \\
\hline Epargne stable & 737925 & 5,9 & 43537 & 1,79 \\
\hline Épargne à terme & 1036608 & 9,6 & 99514 & 4,08 \\
\hline Emprunt à la Fédération & 0 & 0 & 0 & 0 \\
\hline Total (b) & 2436299 & 6,05 & 147423 & 6,05 \\
\hline Marge brute (total (a)-total (b)) & 95995 & & 140796 & 5,78 \\
\hline
\end{tabular}




\subsubsection{Le trop-perçu net}

Le trop-perçu net avant impôt s'obtient en ajoutant à la marge brute les autres revenus (Ex: les frais de perception). De ce résultat il faut soustraire les dépenses administratives ainsi que les dépenses directes des dépôts et des prêts. Au Point de services, les revenus divers se chiffrent à $17 \quad 785$ \$. Les dépenses administratives sont de l'ordre de 72 559; et les dépenses directes des dépôts et des prêts totalisent $36788 \$$.

Le trop-percu net est donc de $49234 \$$, ou $2,02 \$$ par $100 \$$ de passif-dépôt. Le tableau suivant illustre ces calculs.

\section{TABLEAU VI}

TROP-PERCU NET, POINT DE SERVICES

AU 31 JUILLET 1986

\begin{tabular}{|c|c|c|}
\hline Identification & Rev/Dépenses & $/ 100 \mathrm{P} / \mathrm{d}$ \\
\hline Marge brute & 140796 & 5,78 \\
\hline Autres revenus & 17785 & 0,73 \\
\hline Total (a) & 158581 & 6,51 \\
\hline Dépenses directe Pr+dépôts & 36788 & 1,51 \\
\hline Dépenses administratives & 72559 & 2,98 \\
\hline Total (b) & 109347 & 4,49 \\
\hline Trop-perçu total(a)-total(b) & 49234 & 2,02 \\
\hline
\end{tabular}




\subsubsection{Le bilan du Point de services}

Pour mieux voir clair dans la situation financière du Point de services nous en avons tracé le bilan.

Ce bilan pro-forma couvre la période des douze derniers mois se terminant le 18 juillet 1986. Le tableau VII qui suit donne les grandes lignes de ce bilan. 


\author{
TABLEAU VII \\ POINT DE SERVICES SAINT-JEAN-EUDES \\ (CAISSE POPULAIRE D'ARVIDA) \\ BILAN PRO-FORMA \\ AU 18 JUILLET 1986
}

$\overline{\text { Actif }}$
I- Disponibilités
1. Argent en main
2. Fonds de Iiquidité
3. Fonds de dépôt à terme
4. Fonds d'investissement

II- Prêts

1. $R / D$ (-provisions)

2. Hypothécaires

III- Immobilisations

(- Amortissements)

IV- Autres actifs

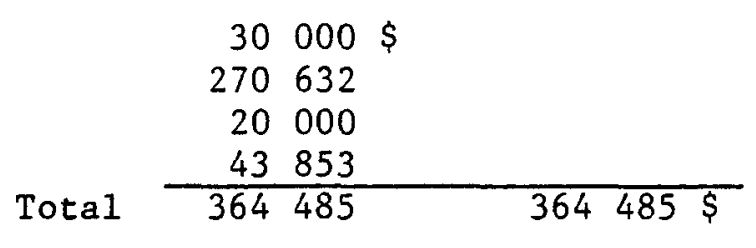

923565

1274244

2197809
2197809

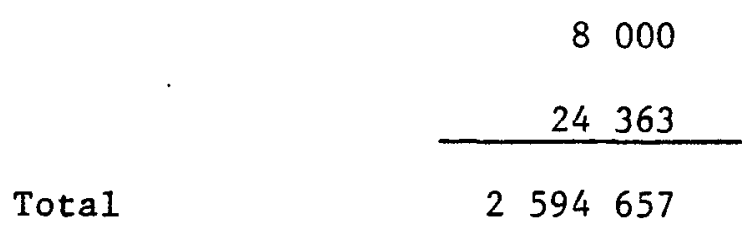

Passif

I- D̂ิ aux membres

1. Capital social

2. $\mathrm{E} O \mathrm{P}$

3. Epargne stable

4. Epargne à terme

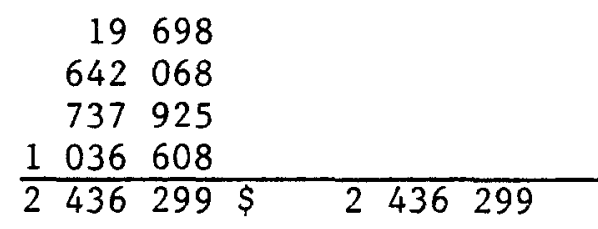

II- Autres éléments du passif

73088

III- Réserve générale

85270

Total

$2594657 \$$ 
Quand on analyse les composantes de ce bilan pro-forma on constate les faits suivants:

- La valeur des dépôts des sociétaires s'établit à 2436299 \$.

- Pour son fonds de roulement quotidien, le Point de services doit posséder dans ses coffres au moins 30000 .

- En vertu des règlements de la Fédération le Point de services devrait se constituer les réserves suivantes.
1) Fonds de liquidité
$270632 \$$
2) Fonds de dépôt à terme.
20000
3) Fonds d'investissement
43853
Tota1
$334485 \$$

- Le fait que le bilan pro-forma n'indique pas d'emprunt à la Fédération tend à prouver que les sociétaires du milieu ne font pas une sur-utilisation de l'épargne par le crédit.

- Des prêts consentis pour 2197809 \$ ont plutôt tendance à signifier que l'épargne du milieu est réinvestie dans le milieu.

\subsubsection{La croissance comparée de l'épargne et du crédit}

Le tableau VIII qui suit établit une comparaison entre le Point de services, le siège social, l'ensemble des banques et des caisses populaires au Québec. Cette comparaison porte sur les trois points suivants à savoir: la valeur de l'ensemble des dépôts, la valeur des 
prêts personnels ou reconnaissance de dettes et la valeur des prêts hypothécaires. Portant sur les années 1982 et 1984 , le tableau nous révèle un ralentissement accentué dans la demande de prêts personnels par les sociétaires du Point de services. Cette demande a chuté de $10,5 \%$ alors qu'elle était en croissance dans les secteurs comparés.

On y constate également que la progression de la valeur des dépôts se situe près de la moyenne de celle des caisses populaires au Québec. Par contre, elle est supérieure de $13 \%$ à celle de la moyenne des banques au Québec.

Quant aux prêts hypothécaires, on s'aperçoit que la croissance de leur valeur est inférieure à celle de la moyenne des caisses. Elle dépasse cependant de $16 \%$ celle de la moyenne des banques.

Aux trois niveaux de comparaison on réalise enfin que la performance du Point de services contribue à affaiblir la performance globale de la Caisse populaire d'Arvida. 
LA POINT DE SERVICES SAINT-JEAN-EUDES

PAR RAPPORT AU SIEGE SOCIAL, CAISSE POPULAIRE D'ARVIDA, CAISSES ET BANQUES AU QUEBEC POUR LES ANNEES 1982 ET 1984

\begin{tabular}{|c|c|c|c|c|c|c|c|c|c|}
\hline N.B. Données en millions & \multicolumn{3}{|c|}{ Dépôts } & \multicolumn{3}{|c|}{ Prêts personnels } & \multicolumn{3}{|c|}{ Prêts hypothécaires } \\
\hline Identification & 1982 & 1984 & $\%$ & 1982 & 1984 & $\%$ & 1982 & 1984 & $\%$ \\
\hline Point de services & 2,01 & 2,32 & 15,4 & 0,86 & 0,77 & $-10,5^{(1)}$ & 0,86 & 1,11 & 29,1 \\
\hline Siège social & 17,47 & 23,52 & 34,6 & 5,51 & 8,54 & 54,9 & 8,97 & 12,45 & 38,8 \\
\hline Caisse populaire d'Arvida & 19,47 & 25,83 & 32,7 & 6,38 & 9,30 & 45,8 & 9,82 & 13,56 & 38,1 \\
\hline Moyenne des caisses au Québec (2) & 7503,00 & 8852,00 & 18,0 & 2. 318,00 & 3225,00 & 39,1 & 6000,00 & 8200,00 & 36,1 \\
\hline Moyenne des banques au Québec (2) & 8800,00 & 8984,00 & 2,1 & 4791,00 & 5520,00 & 15,2 & 4500,00 & $51.00,00$ & 13,3 \\
\hline
\end{tabular}

Note $1=$ En 1985, les prêts personnels ont été de 0,88 millions pour une croissance de $2,3 \%$ sur 1982 .

Note $2=$ Données extraites d'un tableau de François Berger dans le bulletin "D'un sourire à l'autre, novembre 1985, Fédération des Caisses populaires du Centre du Québec. 


\subsubsection{Les indicateurs de Marketing}

Nous notons ici que les quelques indications nous apparaissant les plus pertinentes.

- Le Point de services est un des rares (peut-être le seul) établissements du réseau Desjardins à ne pas s'identifier à l'extérieur par une enseigne lumineuse aux couleurs de Desjardins et portant le sigle du mouvement.

- Les taux en vigueur sur les prêts et les dépôts à termes ne sont pas indiqués comme c'est le cas au siège social.

- Aucune indication ne renseigne le client pour lui dire où se procurer les services spécialisés d'épargne et de crédit; et quels sont ces différents services.

- Lorsque la Caisse organise une promotion spéciale d'un service spécialisé, comme par exemple la campagne MULTI-PRETS EXPRESS, le Point de services affiche les mêmes encarts publicitaires.

\subsubsection{Les indicateurs de personnel}

- Actuellement le Point de services ne requiert que le temps de $13 / 4$ caissières pour assurer son fonctionnement quotidien. Sur 1'heure du dîner, les jours d'affluence, une caissière vient remplacer les deux autres à tour de rôle pour une durée totale de trois heures par jour. 
- Le matin avant $1^{\prime}$ ouverture du Point de services, la caissière à temps complet est la seule à se présenter au Siège social pour préparer le matériel et $\mathrm{y}$ recevoir les directives spéciales. Donc pas de communication directe entre les caissières à temps partiel et le directeur-adjoint aux services courants et administratifs.

- Il n'y a pas, sur place, de personnel cadre supérieur ou intermédiaire pour diriger les opérations du Point de services et assurer les services apécialisés aux sociétaires.

Les caissières, par nécessité, doivent accomplir certaines tâches qui rélèvent normalement d'une commis sénior ou de la chef d'équipe. Ce sont:

1) L'inspection des locaux

2) Face à la réserve: le contrôle de l'encaisse

3) L'envoi de numéraire

4) Le classement de communiqués et circulaires

5) La préparation du dépôt extérieur

6) L'aspect marketing

\subsubsection{Les indicateurs de productivité (production)}

- A certains moments, la propreté des locaux laisse à désirer.

- L'espace d'attente des clients est restreint et parfois les files d'attente vont jusque dehors.

- A l'arrière du comptoir, il y a un bureau inoccupé. 
- Les services spécialisés d'épargne et de crédit normalement offerts dans une caisse populaire sont absents.

- Le système de communication audio avec les sociétaires est déficient.

- Le mur anti-balles nuit à la bonne circulation de l'air climatisé.

- Avec $13 / 4$ caissières on réussit à drainer pour près de 1,5 millions de dollars d'épargne; ceci, sans compter l'épargne à terme qui se fait au siège social de la Caisse.

- Le comptoir comprend trois guichets de services dont un n'est jamais utilisé.

- Les caissières travaillent sans craindre la venue de voleur; ceci est dû à la présence du mur anti-balles.

- Il y a une diminution sensible du nombre de sociétaires. Le tableau III de la page 17 nous indique que, entre 1982 et 1985, le nombre de détenteurs de parts sociales est passé de 1556 à 1092 pour une diminution de $29,6 \%$.

Depuis le début de cette recherche, plusieurs problèmes ont été portés à notre connaissance. Les pages précédentes ont esquissé avec une précision succincte la nature de ces points critiques. Avec l'analyse des indicateurs nous terminons donc le processus de description des principaux éléments permettant l'identification de ces situations problématiques pour lesquelles il faudrait envisager des solutions. 
Dans la partie qui suit et qui met un terme au chapitre II, nous allons procéder à une sélection des problèmes les plus évidents décelés au cours des observations antérieures. A travers cette première sélection, nous arrêterons notre choix sur la problématique pour laquelle nous tenterons d'élaborer une solution dans le chapître III.

\subsection{SÉLECTION D'UNE PROBLEMATIQUE A ETUDIER}

En première sélection nous avons retenu trois situations problématiques. Ce sont: l'achalandage, le marketing et la productivité. La section 2.6.1 trace un résumé-synthèse de chacune.

\subsubsection{Les situations problématiques}

\subsubsection{L'achalandage}

Lorsqu'on parle de l'achalandage au Point de services, deux difficultés s'offrent à notre réflexion pour étude et solution.

\section{1) Les files d'attente}

Les habitudes des sociétaires du Point de services se décrivent ainsi. Les jours d'affluence, les premiers arrivés se répartissent sur deux files, chacune étant face aux guichets un et deux. Quand les dix premiers clients se sont placés sur ces deux lignes, la file d'attente du guichet un se prolonge jusque dans la porte. Les clients suivants se voient donc dans l'obligation de couper cette file d'attente pour atteindre celle du guichet deux, laquelle pourrait s'allonger davantage. Ou encore, le sociétaire demeure tout simplement à la firı de la première file, se retrouvant ainsi dans le portique même dehors. 
En étudiant le plan de l'aménagement physique des lieux à la figure III page 47, on peut aisément visualiser le phénomène de blocage qui survient s'il y a affluence de clients. Une distance de seulement 10 pieds sépare la porte extérieure du guichet un.

Toujours en période d'affluence, les clients du guichet deux contraignent quelques clients de la file d'attente numéro un à se déplacer lorsqu'ils veulent quitter.

2) Le stationnement

D'une capacité maximale de 15 espaces, le terrain de stationnement n'en offre que 13 aux sociétaires quand les deux caissières en ont pris chacune un. L'engorgement de la section avant se produit très rapidement en période d'affluence. En effet, pour peu que les aires de stationnement numéros $3,4,5,7$ et 9 soient occupées, il devient impossible de garer une voiture dans les espaces $1,2,6$, et 8 . La même impossibilité se vit si la voiture veut quitter. La figure IV de la page 43 laisse facilement deviner cette situation. De plus il peut arriver, (cela s'est produit de fait) qu'une automobile encombre les quatre espaces du milieu $(6,7,8,9)$ si elle est mal garée. L'encombrement du stationnement survient simultanément à celui des files d'attente.

\subsubsection{Le marketing}

A l'extérieur du Point de services, l'enseigne lumineuse ne crée pas le sentiment d'appartenance au mouvement Desjardins. Le nom est très bien indiqué; mais les couleurs et le logotype de Desjardins sont totalement absents de notre vision. 
Dans la plupart des institutions bancaires, on affiche bien en vue des clients les différents taux d'intérêt en vigueur. Au Point de services, les taux affichés datent de fort longtemps et ne correspondent plus à la réalité du temps. De plus, ces renseignements ont été placés tout au fond de la salle d'attente des sociétaires. Il faut vraiment faire une démarche spéciale pour en prendre connaissance.

Un nouveau sociétaire, qui se rendrait au Point de services en vue de négocier un placement à long terme ou un emprunt quelconque ne saurait obtenir des informations claires, sans attendre son tour et passer par les guichets de caisse. Les renseignements précisant qu'il faut se présenter au siège social de la Caisse pour profiter de ces services sont pẹ explicites et fort mal localisés. Et c'est surtout lorsqu'il y a promotion spéciale de services comme la campagne MULTI-PRETS EXPRESS, que la présence d'affiches indicatrices renseignant sur les endroits où on peut bénéficier de ces services acquiert toute son importance et sa nécessité. De ce fait à celui de prétendre qu'il n'y a pas de bons services dans cette caisse populaire, le pas n'est pas long à franchir. Et il est aussi de bon ton d'ajouter que cela a contribué pour une certaine part à la diminution du nombre de sociétaires au Point de services entre les années 1982 et 1985 .

\subsubsection{La productivité}

Les ratios de la marge brute et du trop-perçu net démontrent une rentabilité certaine existant au Point de services. Mais une analyse de sa croissance tend plutôt à prouver une faiblesse de productivité. 
Le tableau VIII en page 69 établit le fait que le Point de services contribue au ralentissement de la croissance de toute la Caisse. Le tableau III en page 17 met en évidence que le Point de services a subi une réduction de son nombre de sociétaires de $29,6 \%$ entre 1982 et 1985 alors que le siège social de la Caisse enregistre une hausse de $12,4 \%$.

Si on examine I'aménagement intérieur des locaux du Point de services (figure III, p.47) quelques constatations viennent ajouter à cette faiblesse de productivité. L'espace alloué aux sociétaires pour $I^{\prime}$ attente au guichet est très restreint par rapport à celui disponible à I'arrière des guichets. Un guichet sur trois n'est jamais utilisé pour donner le service à la clientèle les jours de grande affluence. Derrière les guichets, nous constatons l'existence d'un bureau dans lequel il serait possible d'aménager un système de services spécialisés d'épargne et de crédit pour les sociétaires de Saint-Jean-Eudes. Pourtant ces services sont centralisés au siège social de la Caisse, même s'ils ont déjà été dispensés au Point de services du temps qu'il était une caisse autonome avant 1973. La structure actuelle du mur anti-balles empêche $1^{\prime}$ instauration de tels services.

Quelques autres faits, plus mineurs ceux-là, sont aussi de nature à provoquer quelque ralentissement de la productivité du Point de services. En exemple, notons que, de chaque côté du mur blindé, la communication entre employées et clients est rendue difficile et peu discrète par un système auto-vox déficient. Les employées ont aussi dénoncé un manque de propreté des locaux, créant du dédain et/ou une démotivation face au travail. 


\subsubsection{La situation problématique retenue pour étude}

Des trois situations problématiques proposées, nous avons retenu pour étude et recherche de solution celle de la productivité. Deux raisons motivent ce choix. Premièrement, nous croyons qu'une diminution d'environ $30 \%$ du nombre de sociétaires, en I'espace de trois ans, annonce une situation critique à laquelle il faut s'attaquer avant quelle ne devienne catastrophique. Deuxièmement, nous sommes enclins à croire qu'une solution au problème de la productivité pourrait entraîner du même coup celles de l'achalandage et du marketing. Le chapître suivant fournira les indications justifiant ce choix et les orientations vers lesquelles nous dirigerons notre proposition de solution.

\section{CONCLUSION}

Tout au long du chapître II, nous avons passé en revue les aspects environnementaux du Point de services. Que ce soit par la description des structures et de l'aménagement physique des lieux, que ce soit par la description de l'environnement ou du processus ou du climat organisationnel, chacun des fondements analysés dans ce chapître aura permis d'identifier un certain nombre de faits ou situations qui sont tous interdépendants. Par l'analyse des principaux indicateurs, notre travail de recherche a permis d'établir des liens de convergences nous amenant à privilégier pour une étude plus approfondie au chapître suivant, le problème de la productivité du Point de services. 
CHAPITRE III

LE SYSTEME PERTINENT PROPOSE:

UN SYSTEME DE PRODUCTION 
CHAPITRE III

LE SYSTEME PERTINENT PROPOSE:

UN SYSTEME DE PRODUCTION

\section{INTRODUCTION}

A la fin du chapître II, nous avons pu établir que le problème le plus important à solutionner au Point de services en était un de productivité. Ainsi donc, au cours du présent chapître, nous entendons introduire un certain nombre de variables permettant de justifier et de définir dans les faits et le temps la solution que nous suggérons aux administrateurs et aux sociétaires de la Caisse Populaire d'Arvidạ.

Le travail se fera en trois phases. La première étape menant à une justification de notre choix, sera appuyée par une revue de 1ittérature. Dans la deuxième partie du chapître, une définition du système approprié de solution sera proposée. Finalement, la troisième partie essaira de démontrer la validité du système d'activités qu'il serait souhaitable d'implanter.

\subsection{LE CHOIX DU SYSTEME PERTINENT}

\subsubsection{Quel système adopter?}

Comme la problématique identifiée au chapître précédent est relative à la productivité, il incombe à L'auteur d'offrir aux décideurs de la Caisse Populaire d'Arvida la possibilité d'instaurer à Saint-JeanEudes un système de production de services amélioré et plus complet. La nouvelle organisation du travail poursuivrait un double objectif. Pre- 
mièrement, elle permettra la dispensation de services d'une qualité supérieure. En deuxième lieu, elle réintroduira au Point de services une gamme de services jadis offerts mais maintenant centralisés au siège social (il faut entendre ici les services spécialisés d'épargne et de crédit). Par voie de conséquence un troisième objectif s'inscrit en corollaire des deux autres soit celui de récupérer une partie des sociétaire perdus, d'en ajouter d'autres pour accroitre ainsi le développement financier et revaloriser la production au Point de services.

\subsubsection{Justification d'un tel système.}

Une étude démographique attentive dans le secteur a fait découvrir les faits suivants. Sur une période de 10 ans, le nombre de familles est passé de 626 à 751 en la paroisse de Saint-Jean-Eudes, soit une augmentation de $19,97 \%$. Pour ce même laps de temps, qui s'étend de 1976 à 1986, la population de la paroisse est passé de 2514 à 2279 résidents, pour une diminution de $9,35 \%$. Le tableau suivant étale dans le temps ces données. 
TABLEAU IX

CROISSANCE DE POPULATION .

PAROISSE SAINT-JEAN-EUDES

\begin{tabular}{|c|c|c|}
\hline ANNEES & $\begin{array}{c}\text { NOMBRE } \\
\text { DE FAMILLES }\end{array}$ & POPULATION \\
\hline 1976 & 626 & 2514 \\
\hline 1980 & 692 & 2400 \\
\hline 1986 & 751 & 2279 \\
\hline écart & +125 & -235 \\
\hline
\end{tabular}

Source: Archives, paroisse Saint-Jean-Eudes

Saint-Jean-Eudes, comme à peu près partout ailleurs au Québec, vit un phénomène notoire de dénatalité. Il y est tellement apparent que la croissance du nombre de familles ne réussit pas à le contrer.

Se borner à prétendre que la diminution du nombre de sociétaires au Point de services est attribuable à la décroissance démographique du secteur rendrait factice toute notre argumentation. Car en supposant que toutes les personnes en moins à Saint-Jean-Eudes étaient des membres de la Caisse, cette chute n'expliquerait qu'un peu plus de $50 \%$ de 1 a perte du nombre de détenteurs de parts sociales. Tel n'est pas le cas puisque la diminution de la population s'étend sur une période de 10 ans alors que celle du nombre de sociétaires s'étale dans un délai de 
quatre ans. Il faut donc chercher ailleurs les raisons expliquant cette faiblesse de productivité du Point de services,

Après sa fusion à la Caisse populaire d'Arvida en 1973, la Caisse populaire Saint-Jean-Eudes, devenu un point de services, a continué à dispenser à ses sociétaires les mêmes services qu'elle leur fournissait lorsqu'elle était une caisse autonome. Ce n'est que durant I'année 1980 que les dirigeants prirent la décision de transférer au siège social de la Caisse tous les services spécialisés d'épargne et de crédit accordés à Saint-Jean-Eudes.

Pour les années 1973 jusqu'à celle de 1982, il serait très onéreux, voire pratiquement impossible, d'évaluer jusqu'à quel point la fusion aurait pu avoir modifié à la baisse le nombre de sociétaires en provenance de Saint-Jean-Eudes. Mais après la disparition des services spécialisés d'épargne et de crédit, on a pu constater une forte diminution du nombre de sociétaires entre les années 1982 et 1985. Le tableau suivant nous montre cette diminution. 
TABLEAU $X$

TAUX DE DECROISSANCE

DU NOMBRE DE SOCIETAIRES

POINT DE SERVICES

\begin{tabular}{|c|c|c|c|}
\hline ANNEE & NOMBRE & REDUCTION & TAUX \% \\
\hline 1982 & 1552 & - & - \\
\hline 1983 & 1253 & 299 & 12,8 \\
\hline 1984 & 1126 & 127 & 10,1 \\
\hline 1985 & 1092 & 34 & 3,0 \\
\hline Total & & 460 & 29,6 \\
\hline
\end{tabular}

Par déduction hypothétique, il est permis d'affirmer que cette réduction du nombre de sociétaires à Saint-Jean-Eudes a été provoquée par la centralisation au siège soclal de la Calsse des services spécialisés d'épargne et de crédit. Il est aussi possible d'expliquer cette affirmation par cette supposition logique: tant qu'à être au Carré Davis, pourquoi ne pas magasiner les taux d'intérêt sur les prêts et. placements à long terme? Cela pouvait se réaliser dans 4 institutions financières concurrentes sans avoir à déplacer sa voiture en chaque occasion. 
Le sondage réalisé à l'été 1985 tend aussi à prouver cette hypothèse. En effet, à une question qui demandait aux sociétaires ce qu'ils feraient si la Caisse en venait à fermer le Point de services, $15,9 \%$ des répondants annoncaient qu'ils changeraient d'institution financière si une telle situation se produisait. Ainsi donc, plus de services à notre caisse populaire, une autre institution financière les fournira. Mais par contre, $84,1 \%$ des répondants ont avoué leur volonté de faire toutes les pressions nécessaires pour garder leur caisse populaire ouverte malgré ses faiblesses.

Enfin, le Point de services appartient aux gens de Saint-Jean-Eudes. Et pour près de $48 \%$ des sociétaires (d'après le sondage de l'été 1985), il représente la seule institution financière avec laquelle ils font affaires. Priver les sociétaires de leur Point de services, c'est comme si on les amputait d'une partie d'eux-mêmes. II faut donc le garder en opération et en améliorer le rendement.

Les écrits sur le monde coopératif tendent à confirmer cette nécessité d'une institution financière coopérative au sein des petites collectivités. Les pages qui suivent, et, qui traitent du rôle des coopératives d'épargne et de crédit dans notre milieu régional visent à établir la preuve de cette nécessité socio-économique des caisses populaires. 


\subsection{REVUE DE LITTERATURE:}

LES COOPERATIVES D'EPARGNE ET DE CRÉDIT DANS NOTRE MILIEU

\subsubsection{Les caisses populaires et le développement régional au Saguenay-Lac-Saint-Jean.}

"Après un décollage économique fulgurant au début du siècle, la région du Saguenay-Lac-Saint-Jean ne parvient pas, depuis la deuxième guerre mondiale, à retrouver un second souffle et à soutenir une croissance économique qui assureraft au moins de l'emploi à toute la population."(1) Les deux entités industrielles qui composent l'axe du développement de notre région ont accaparé la presque totalité du potentiel économique de ce coin de pays. Et, s'identifiant à $1^{\prime}$ industrie de l'aluminium et à celle des pâtes et papiers, le Saguenay-Lac-Saint-Jean est vite devenu tributaire de ces deux types de production. La capitale de 1'aluminium (Arvida) (devenue Jonquière) et Kénogami (devenue Jonquière) la ville du papier ont presque toujours figuré en tête d'affiche de la publicité touristique de la région.

L'Alcan et les compagnies de pâtes et papier, après avoir joué un rôle moteur pour l'accroissement du produit réel global de la région et pour 1'amélioration des infrastructures industrielles, ont été incapables de déclencher une dynamique de développement régional.(2)

1- Prévost, Paul, Du développement coopératif au développement régional, Revue Prothée, vol. VIII, no 3, aut. 1980, p.12.

2- Fournier, Claire, La prise de décision dans les coopératives et le développement régional, Revue Prothée, Vol. VIII, No. 3, Aut. 1980 p.33. 


\begin{abstract}
Mais en raison de la dispersion de leurs activités multinationales de production, ces deux fondements de base de notre industrie ont été plutôt nocifs face à "l'émergence de structures bien articulées au Saguenay-Lac-Saint-Jean."(3) Plusieurs études sur les économies régionales ont cependant mis en évidence que, de tous les intervenants dans le développement économique de notre région, le modèle coopératif soit celui qui offre la meilleure possibilité de générer chez nous un développement régional auto-centré.
\end{abstract}

Les socialistes utopistes, les pionniers de Rochdale et leurs disciples ont en effet développé des bases idéologiques qui pourraient aujourd'hui inspirer entre autres la conception d'un modèle de développement régional typiquement coopératif. (4)

Lui-même inspiré par I'histoire des pionniers de Rochdale, Alphonse Desjardins a toujours prôné que c'est dans le milieu et pour le milieu que la coopérative d'épargne et de crédit doit trouver ses racines et sa source de vie.

3- Fournier, Claire, La prise de décision dans les coopératives et le développement régional, Revue Prothée, Vol VIII, no 3. Aut, 1980, p.33

4- Prévost, Paul, La coopération et le développeemnt régional, Chicoutimi, LEER, 1981, p.3. 
La Caisse populaire sera le foyer où s'élaboreront les les projets d'organisation, et les buts à atteindre sont nombreux; elle fera éclore presque spontanément... des initiatives de toutes sortes;... Son succès inspirera confiance et enhardira les gens à tenter d'autres réalisations économiques, et de ces conceptions nouvelles jaillira un ensemble d'activités collectives des plus fécondes. (5)

Cependant, il voyait en chacune une faiblesse: une insuffisance notoire à fournir certains services qu'un développement régional accentué rendrait indispensables. C'est pour cette raison qu'il a lancé un projet de création d'une Fédération visant à harmoniser les activités multiples des caisses locales et devenant ainsi un centre initiateur pour activer les diverses créations locales.

De sa création coopérative, Alphonse Desjardins disait qu'elle possédait "une irrésistible force conquérante" qui " ne manquera pas d'être un grand facteur de l'évolution qui s'accomplit dans le monde économique..."(6). Or depuis "quelques années au Québec, le système coopératif nous est présenté comme la voie privilégiée de la prise en main de notre devenir économique et comme l'instrument de la participation de la population à ce projet de développement". (7)

5- Desardins, Alphonse, Réflexion d'alphonse Desjardins, Lévis, Confédération des Caisses populaires et d'économie Desjardins du Québec, 1986, p.71.

6- Desjardins, Alphonse, 0p. cit., p.36

7- Audet, Réjean, Les coopératives financières et le développement régional, revue Prothée, vol VIII, no 3. Aut. 1980 p.70. 
Dans un modèle de développement régional, les institutions financières en constituent un élément fondamental. En effet, pour que de telles institutions jouent pleinement leur rôle de moteur et développeur de l'économie régionale, il leur faut remplir dans le milieu ces deux fonctions:

1) "Intégration dans le milieu régional des opérations de mobilisation de 1 'épargne."

2) "Intégration dans le milieu régional des opérations d'allocation: financement de la consommation et financement de 1 'investissement."(8)

D'accessibilité facile, la caisse populaire réalise ce "double caractère de déversoir d'épargne et de crédit» (financement)" ... puisqu'elle fonctionne dans un milieu restreint, la paroisse;... C'est grâce à elle que l'épargne du peuple peut être mise à la disposition du peuple, lui être doublement avantageuse en aidant ses initiatives..."(9). La caisse populaire, tout comme "L'organisation coopérative, c'est la formule par laquelle individuellement et collectivement, on cherche à être de plus en plus propriétaire de l'économie, et maître de sa propre activité économique de production de service et de consommation.»(10).

8- Idem, p. 3

9- Desjardins, Alphonse, Op. cit., p.48

10- Bernier, Guy, Entre nous, revue Ma Caisse, vol. 23, no 7. nov-déc. 1986 , p. 4 . 
De cette façon, l'istitution financière coopérative qu'est la caisse populaire devient un "levier essentiel afin de réaliser cet auto-développement»(11) régional.

Dans un autre ordre d'idées, il est reconnu et prouvé par des spécialistes en développement économique et des données statistiques que 1a PME crée plus d'emplois que la grande entreprise. Pour cette raison, les principaux agents économiques de la Sagamie ont entrepris de faire la promotion de la PME et d'en soutenir la création. On a donc mis sur pied une société capable de venir en aide aux personnes désireuses de démarrer des PME manufacturières. Les caisses populaires via leur Fédération régionale, se sont impliquées en devenant un partenaire majeur de la Société en commandite de création d'entreprises (Socrent), y injectant une somme de $1000000 \$$.

Enfin, des études démographiques récentes ont démontré que la population du Saguenay-Lac-Saint-Jean profitait d'un taux de croissance supérieur à 11\% (en 1985) depuis 1971. De 295500 habitants qu'elle était en 1985, cette population devrait atteindre 308300 en 1990 pour une croissance totale de $16,08 \%$ (comme le démontre le tableau suivant) par rapport à 1971.

11- Audet, Réjean, Op. cit, p. 71 
TABLEAU XI

POPULATION

DU SAGUENAY-LAC-SAINT-JEAN

\begin{tabular}{|l|c|c|c|}
\hline & Population & $\begin{array}{c}\text { Importance } \\
\text { relative } \\
\text { au Québec }\end{array}$ & $\begin{array}{l}\text { Indice de } \\
\text { croissance }\end{array}$ \\
\hline Année & Nbre & $\%$ & $1971=100$ \\
\hline 1971 & 265600 & 4,41 & 100,00 \\
1976 & 269000 & 4,31 & 101,28 \\
1981 & 285300 & 4,43 & 107,42 \\
1982 & 289700 & 4,47 & 109,07 \\
1983 & 292300 & 4,48 & 110,05 \\
1984 & 293900 & 4,49 & 110,66 \\
1985 & 295300 & 4,49 & 111,26 \\
1986 (p) & 298700 & 4,51 & 112,46 \\
1987 (p) & 301200 & 4,53 & 113,40 \\
1988 (p) & 303700 & 4,54 & 114,34 \\
1989 (p) & 306100 & 4,55 & 115,25 \\
1990 (p) & 308300 & 4,56 & 116,08 \\
\hline
\end{tabular}

(p): Prévision.

Source: C.C.P.E.D.Q., Direction Recherche Division Analyse économique Octobre 1986 
De cette population de 295500 habitants en 1985,168197 personnes possédaient au moins un compte d'épargne dans une caisse populaire Desjardins. Cette présence de Desjardins dans notre milieu régional signifie un taux de pénétration de $56,9 \%$; ce qui démontre une croissance de près de $8 \%$ par rapport à 1982 . Il s'agit aussi d'un taux de pénétration supérieur à la moyenne québécoise pour la même année (Voir le tableau suivant).

TABLEAU XII

TAUX DE PENETRATION DES CAISSES POPULAIRES

FEDERATION DU SAGUENAY-LAC-SAINT-JEAN ET CONFEDERATION

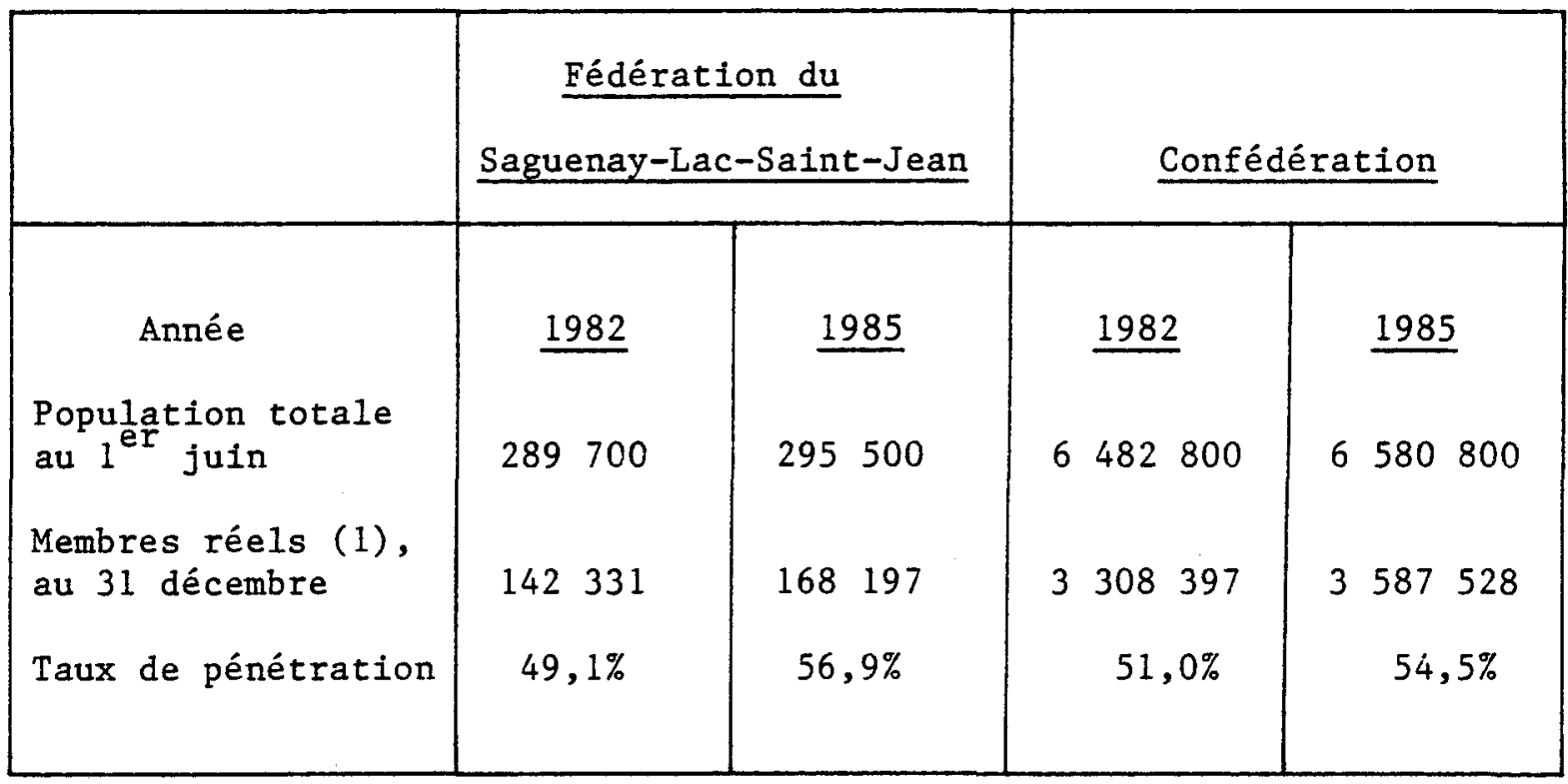

(1) Afin de permettre la comparaison entre 1982 et 1985 , les membres des caisses d'économie dont le nombre n'était pas disponible en 1982 ont été exclus du nombre de membres réels de la Confédération.

Source: C.C.P.E.D.Q., Direction Recherche, Division Analyse économique et Division Analyse marketing, octobre 1986. 
Une étude sur la présence du mouvement coopératif au Québec, publiée en 1979, revélait que la région du Saguenay-Lac-Saint-Jean était desservie par 78 caisses d'épargne (65 caisses populaires Desjardins, 6 caisses d'entraide, 6 caisses d'économie, une caisse d'établissement). Celles-ci étaient localisées dans 53 des 70 municipalités de la région et elles offraient des services à $90,77 \%$ de la population (257 340 h) . Quant aux banques, on en comptait 56; elles étaient établies dans 12 municipalités et offraient services à 214594 personnes. Le tableau XIII de même que l'histogramme qui suivent démontrent le très grand souci des caisses populaires de vouloir répondre aux besoins d'une collectivité régionale la plus étendue possible. Au Saguenay-Lac-Saint-Jean, les caisses populaires sont présentes dans plus de $75 \%$ des municipaliltés. 
TABLEAU XII

RÉPARTITION DES CAISSES PAR MUNICIPALITE

SELON L'IMPORTANCE DE LEUR POPULATION

SAGUENAY-LAC-SAINT-JEAN

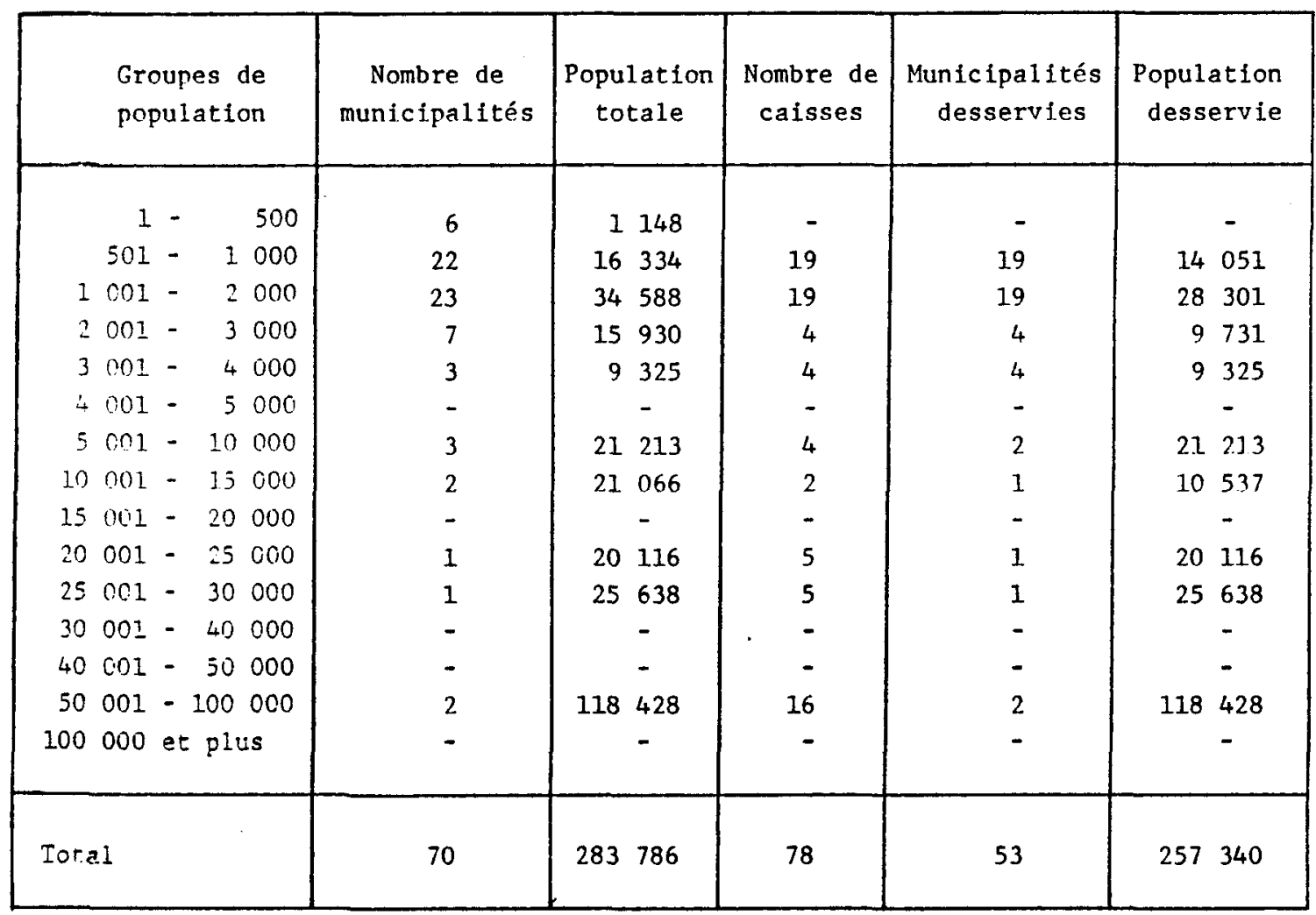

Source: Compilation du service de la recherche, MCCIF, dans Profil du Mouvement coopératif au Québec, OPDQ, 1977 p.85 
HISTROGRAMME

NOMBRE D'INSTITUTIONS PAR MUNICIPALITE

SUIVANT LES GROUPES DE POPULATION

SAGUENAY-LAC-SAINT-JEAN

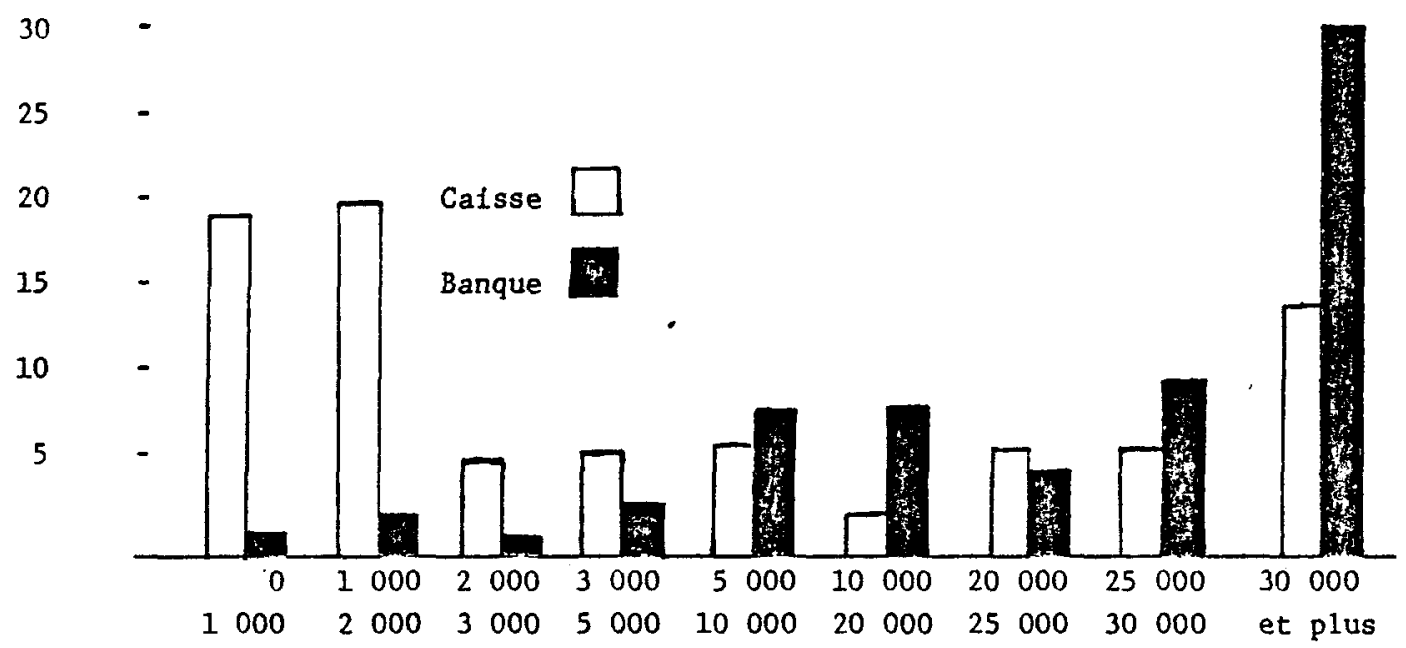

Source: Profil du mouvement coopératif au Québec OPDQ 1979 p.85

FIGURE VIII 
Quand des renseignements de régie interne à la Fédération nous indiquent que l'institution financière la plus concurrentielle jouit d'un taux de pénétration d'environ $20 \%$ seulement, quand des données statistiques nous apprennent que les caisses populaires sont présentes presque partout, même dans de très petites collectivités municipales, il est permis d'affirmer qu'elles comptent parmi les agents de développement régional les plus importants.

\subsubsection{Le rôle social des caisses populaires Desjardins}

Beaucoup d'auteurs qui ont élaboré des écrits sur les activités coopératives sont unanimes à dire qu'"une coopérative est d'abord et avant tout un service que les gens veulent se donner."(12) Celle-ci "s'implante par une volonté collective née d'un besoin ressenti par une communauté."(13) Ainsi donc,

L'action coopérative $n^{\prime}$ a pas pour but de distribuer occasionnellement de l'aide pour soulager des misères ou promouvoir des causes nobles. L'action coopérative part de plus loin et vise plus loin. Ce qu'elle cherche, c'est à rendre les gens plus autonomes et libres en leur fournissant les moyens de se prendre en mains et de répondre euxmêmes, seuls et en groupe, à leurs propres besoins.(14)

12- Dallaire, Rosemarie, Trudel Dominique, La problématique actuelle et les perspectives d'avenir dans les coopératives, Chicoutimi, LEER, automne 1979, P. 25.

13- Idem, p. 25.

14- Blais, Raymond, Message du président à 1'assemblée générale du M.C.P.E.D. en mars 1986 , Lévis, Confédération des Caisses populaires et d'économie Desjardins, 1986, Dossier 12, p.12. 
Si M. Raymond Blais parlait ainsi dans son message annuel de 1986, c'est qu'il voulait insister sur l'objectif ultime des caisses populaires, tel que le prônait le fondateur lui-même, soit: "Solutionner la question sociale sur le terrain économique."(15) Face à cet objectif poursuivi par le fondateur, la caisse populaire apparaît comme une collectivité instituée pour compenser la faiblesse de chaque individu composant la communauté économique. Quelques principes fondamentaux d'organisation deviennent absolument importants et essentiels pour l'implantation d'une telle collectivité. Ce sont: la primauté de la personne sur le capital, le caractère local et restreint de la collectivité et le contrôle par des gens du milieu, tout cela pour une accessibilité facile et constante.

Pour Alphonse Desjardins, la mise en place de ces principes organisationnels a justifié le caractère paroissial et/ou municipal qu'il a voulu donner à sa coopérative d'êpargne et de crédit ainsi qu'à toutes celles qui ont suivi. En 1986, ce point de vue d'Alphonse Desjardins, Raymond Blais, alors président du M.C.P.E.D. I'exprimait ainsi:

15- Desjardins, Alphonse, Op. cit, p. 15. 
L'action coopérative, en somme, c'est une méthode d'organisation des activités économiques par laquelle un groupe de personnes tente, dans le respect mutuel et la confiance réciproque, de rendre ces activités le plus équitables, le plus appropriées, et le plus efficaces possible dans $I^{\prime}$ intérêt de tous les partenaires et de chacun d'eux, et dans la conformité aux exigences du bien commun de leur collectivité. Dès lors qu'on saisit la portée de cette finalité on comprend mieux la complicité fraternelle que la formule coopérative entretient avec $I^{\prime}$ action bénévole et les personnes, groupes, associations qui l'encouragent, I'organisent et la réalisent.(16)

Par cette déclaration, il confirmait également que "I'association d'épargne et de crédit est... I'âme de toutes les autres parce qu'elle est la meilleure éducatrice et réunit les fonds qui, sous forme de prêts, viendraient féconder les efforts des autres associations." (17)

Convaincu qu'il était que la croissance d'un peuple se réalise par le développement de petites communautés solides capables d'initiatives et d'entraide mutuelle, Alphonse Desjardins en a cimenté le caractère local des caisses dans I'action bénévole de ses dirigeants. II ne pouvait en être autrement car une communauté se développe par des gens du milieu, qui connaissent le milieu et auxquels on peut communiquer les besoins de ce même milieu. L'action bénévole était le moyen privilégié pour y arriver parce qu'elle est féconde, novatrice et productrice.

16- Blais, Raymond, Le bénévolat, Lévis, Confédération des Caisses populaires et d'économies Desjardins, 1986, Dossiers 10 p.3.

17- Desjardins, Alphonse, Op. cit. p. 31. 
Ainsi le soulignait Raymond Blais dans son commentaire sur le bénévolat: "Une oeuvre mise sur pied dans I'indigence et le dévouement rend de tels services que la communauté en vient à la prendre en charge, soit par le biais de ses institutions collectives, soit par l'intégration de I'initiative à des champs organisés de 1'action communautaire."(18) Le mouvement des caisses populaires et d'économie Desjardins s'est développé de cette façon et il a été pris en charge par les Québécois. Encore aujourd'hui,

... les dirigeants doivent être représentatifs de leur milieu. Ils sont le prolongement de la caisse et jouent un rôle clé dans l'information dont a besoin la caisse avant d'accorder certains prêts et faire le suivi de certains financements... les trois conseils de bénévoles que possèdent les caisses sont une ressource inestimable...(19)

car le bénévolat est gage d'une action sociale saine, adaptée aux besoins du milieu.

Sa dernière année comme président du M.C.P.E.D., Raymond Blais I'a consacrée à prêcher un retour aux sources, retour aux objectifs et valeurs véhiculés par le fondateur. Toute sa prédication s'est faite en mettant I'accent sur l'action bénévole des membres et le rôle social du M.C.P.E.D. Une des premières données fournies par le rapport statistique du M.C.P.E.D. pour 1'année 1985 fut pour souligner que Desjardins, ce sont des gens d'abord. Chez Desjardins, on compte environ 3 dirigeants

18- Blais, Raymond, le bénévolat, Lévis, Confédération des Caisses populaires et d'économies Desjardins, 1986, Dossiers 10, p.3.

19- Gagné, Jean-Paul, $14^{\text {e }}$ congrès du mouvement Desjardins du 28 au 30 novembre, Caisses pop: redonner la priorité aux valeurs coopératives, Journal Les Affaires, Samedi 22 novembre 1986. 
bénévoles par 4 employés; à la grandeur du Québec, les 18600 bénévoles et les 24088 employés fournissent de I'aide à plus de 4 millions de membres (volr le tableau suivant).

TABLEAU XIV

DESJARDINS: : DES GENS D'ABORD

\begin{tabular}{|c|c|c|c|}
\hline & 1985 & 1984 & $\Delta$ \\
\hline $\begin{array}{l}\text { Folios } \\
\text { Membres }\end{array}$ & $\begin{array}{lll}5 & 141 & 000 \\
4 & 030 & 000\end{array}$ & $\begin{array}{lll}5 & 014 & 000 \\
3 & 975 & 000\end{array}$ & $\begin{array}{r}128000 \\
55000\end{array}$ \\
\hline Dirigeants & 18600 & 18700 & -100 \\
\hline $\begin{array}{l}\text { Employés caisses } \\
\text { Employés institutions }\end{array}$ & $\begin{array}{r}20853 \\
3 \quad 235 \\
24088\end{array}$ & $\begin{array}{r}20487 \\
2973 \\
23460\end{array}$ & $\begin{array}{l}366 \\
262 \\
628\end{array}$ \\
\hline
\end{tabular}

Source: Statistiques des résultats des Calsses Desjardins en 1985, Dossier 11, 1986 Confédération des Caisses populaires et d'économies Desjardins.

Dans un contexte difficile au niveau de la création de l'emplo1, les caisses populaires ont réussi à créer 628 nouveaux emplois en 1985. Cette performance, reflet d'une mission socio-économique bien remplle, découle pour une bonne part du travail des dirigeants bénévoles: ceux-ci étant guidés non par la recherche du profit maximum mais par la recherche d'une rentabilité efficace pour assurer un plus grand nombre et." de meilleurs services à une collectivité de plus en plus grande et de tout niveau social. Au Saguenay-Lac-Saint-Jean come à la grandeur du Québec, les dirigeants des caisses ont réalisé que la rcoopérative, comme 
toute autre entreprise, ne peut plus assumer un rôle social uniquement envers ses membres et ses employés, mais se doit d'oeuvrer en tant que bon citoyen au sein de l'environnement qui est le sien."(20)

Chez nous, dans la région de la Sagamie, la Fédération des caisses populaires Desjardins a su développer son rôle social. En effet, en 1987 et pour la neuvième année consécutive, elle organise par l'intermédiaire de chacune des caisses le concours international des jeunes. Très régulièrement, une caisse prête ses murs pour permettre à un artiste local de faire connaître ses oeuvres. Chaque caisse imprègne son nom et celui de Desjardins dans différentes collectivités régionales par de multiples dons destinés à aider ces groupements (bourse d'étude pour étudiant du Cégep local, don à la Coop funéraire du Saguenay pour l'ouverture d'une nouvelle salle d'exposition, don d'un magnétoscope au foyer Georges Hébert, don substantiel au Café Chrétien d'Arvida; tout cela par la Caisse populaire d'Arvida).

A l'instar de plusieurs dirigeants et directeurs de caisses interviewés par Jean-Paul Gagné du journal Les Affaires, on peut affirmer qu' Une caisse c'est une banque plus. Le fait d'être une coopérative nous oblige à être davantage aux aguets des besoins du milieu."(21) En

20- Beaulieu, Rémy, Le bilan social en milieu coopératif, Chicoutimi, LEER, Automne 1983. p. 39.

21- Gagné, Jean-Paul, 3400 dirigeants de caisses se réusissent en congrès. Le défi des caisses pop: concilier valeurs coopératives et efficacité, journal Les Affaires, Samedi 22 novembre 1986, p. 10. 
fait, à la caisse populaire, on a compris depuis longtemps qu'il ne faut plus ignorer les sollicitations dont on est 1'objet. On y répond non pas seulement sur une base de rentabilité économique mais aussi sur une base de rentabilité sociale. "II ne se passe pas une journée sans qu'un directeur de caisse soit appelé à répondre à un besoin particulier d'un membre ou d'un organisme du milieu."(22)

La population connaft sa caisse populaire; elle connait sa capacité de services; et elle exige sa présence dans le milieu. Pour cette raison et "Pour consacrer leur mission sociale, de plus en plus de caisses populaires publient un bilan social, parallèlement à leur bilan financier. Les caisses sont invitées à cet exercice par la Confédération des caisses populaires Desjardins et les fédérations de caisses populaires qui, pour la plupart, ont commencé à publier des bilans sociaux." (23)

22- Idem, p. 11 .

23- Idem, p. 11 . 
3.2.3 Comment la caisse populaire réalise son action socio-économique

Dans un témoignage rapporté par Monique Rioux dans la revue Ma caisse, Henri Allard, administrateur à la caisse populaire de 1'Auvergne, disait que

\begin{abstract}
C'est à travers la coopérative que nous pourrons élaborer une société qui ressemble le plus à nos aspirations humaines. Parce que, dans l'ère où nous vivons actuellement, il me semble que l'individualisme ne répond pas réellement aux désirs intérieurs des gens. C'est pourquoi Desjardins doit se dépêcher de ranimer les valeurs coopératives pour donner un nouvel élan à la société... Nous évoluons sur une base de valeurs acquises mais il faut les ranimer. (24)
\end{abstract}

Si "La formule coopérative repose entièrement sur la conception que la personne est un être libre et social aspirant au bien-être et au bonheur."(25), la déclaration de Henri Allard signifie que l'action coopérative doit reposer sur des valeurs solides et bien vivantes. La présence de ces valeurs fondamentales garantit à la caisse qu'elle sera en mesure d'atteindre pleinement ses objectifs économiques et sociaux. Quand Alphonse Desjardins eut l'idée et qu'il eut lancé la première caisse populaire, il désirait que l'économie soit mise au service de I'humain. Maintenant, "Chez Desjardins, cette visée peut se structurer autour de cinq valeurs centrales "(26) qui sont:

24- Giroux, Martine, Témoignage, revue Ma caisse, nov-déc, 1986, vol. 23 , no 7, p. 15 .

25- Collaboration, Les valeurs permanentes du mouvement Desjardins, revue La caisse, nov-déc 1986, vol 23, no 7, p.12.

26- Idem, p. 12 . 
1) L'argent au service du développement humain.

2) L'engagement personnel.

3) L'action démocratique.

4) L'intégrité et la rigueur dans l'entreprise coopérative.

5) La solidarité avec le milieu.(27)

Pour démontrer comment ces cinq valeurs de base permettent à une caisse de bien remplir sa mission économique et sociale, quelques mots d'explications sur chacune s'avèrent nécessaires.

\subsubsection{L'argent au service de l'humain.}

A cause de la primauté des personnes dans une coopérative d'épargne et de crédit, l'argent (c'est-à-dire le profit) n'est pas un objectif à atteindre. Il constitue plutôt un moyen à la disposition de leurs projets socio-économiques. Ainsi donc, la caisse populaire vise à fournir des services financiers les plus diversifiés et nombreux possible; les offrir au plus grand nombre de personnes possible, chacune ayant une chance égale de bénéficier de ces services; les offrir à des coûts acceptables et avantageux pour tous et chacun. Enfin, ce sont les membres eux-mêmes, par l'action démocratique, qui définissent de quelle façon leur caisse peut le mieux répondre à leurs besoins personnels et communautaires.

27- Idem, pp $12-14$ 


\subsubsection{L'engagement personnel.}

"La volonté de se prendre en main conduit au partage réel des moyens d'action dont chacun dispose avec la certitude que chaque personne sera traitée également dans les processus décisionnels et équitablement dans le partage des résultats.»(28) Cette volonté de se prendre en main ne peut se réaliser sans un engagement personnel des membres, des dirigeants et des employés de la caisse. Les gestes financiers de tous ces gens contribuent à la formation d'un patrimoine collectif important.

"La mise en commun des ressources et l'action concertée" (29) des membres sont accentuées par la possibilité que chacun participe aux décisions, y participe par un engagement personnel bénévole comme dirigeant. Cela se traduit par des services mieux structurés et de qualité.

Enfin, au personnel employé, Desjardins propose un triple engagement: engager son savoir-faire, sa loyauté et sa fidélité.

\subsubsection{L'action démocratique.}

La pratique coopérative en ce domaine s'appuie sur la liberté humaine et fait appel à 1'intelligence et à la volonté des coopérateurs, dirigeants élus et membres du personne1, pour obtenir des résultats durables. En ce sens, les mécanismes démocratiques de Desjardins, bien loin de constituer des contraintes, sont des facteurs importants de son succès. (30)

$$
\begin{aligned}
& 28-\text { Idem, p. } 12 \\
& 29-\text { Idem, p. } 12 \\
& 30-\text { Idem, p. } 13
\end{aligned}
$$


Le principe, un membre $=$ un vote, consacre ce caractère de démocratie et lui confèré cette valeur d'universalité absolument essentielle aux "gens qui veulent participer aux décisions qui les concernent."(31) Le sens de l'écoute et de la communication, le leadership, la nécessité de la concertation et une solidarité permanente représentent les quatre dimensions autour desquelles se construit la méthode démocratique de Desjardins.

3.2.3.4 L'intégrité et la rigueur dans 1'entreprise coopérative.

"L'existence même des institutions financières repose sur la confiance que leur accordent les consommateurs... C'est pourquoi l'intégrité de toutes les personnes qui ont un rôle à jouer dans l'administration et la gestion des fonds est une valeur de base."(32) Dans une coopérative d'épargne et de crédit,

l'intégrité prend aussi la forme du respect de ses engaments financiers et de la parole donnée et déborde du cadre de la stricte éthique professionnelle. C'est le fondement même des rapports entre les membres et les groupes et, de ce fait, le ciment de leur solidarité...

De plus, le fait que les caisses et les diférents organismes Desjardins soient des entreprises collectives nécessite une transparence et une rigueur sans faille. Gérer l'argent des membres est une responsabilité qui ne tolère pas l'à-peu-près et qui requiert les méthodes les plus efficaces. (33)

31- Idem, p. 13.

32- Idem, p. 13.

33- Idem, p. 13. 


\subsubsection{La solidarité avec le milieu}

Si les membres ont toujours bien supporté leurs caisses "C'est qu'ils ont vu en elles un foyer de développement sous leur contrôle.»(34) Les caisses ont bien vite compris ce rôle que leurs membres attendaient d'elles; elles n'ont donc pas hésité à encourager de leurs appuis humains et financiers les initiatives locales collaborant ainsi avec des groupes qui partageaient "le même idéal coopératif."(35) Les caisses populaires sont nées au Québec pour les Québécois. Elles ont su promouvoir et développer cette "complicité entre la population, les membres, leurs caisses et leur mouvement" laquelle "se traduit par une collaboration constante avec l'Etat québécois»(36) tout en se gardant bien de s'identifier à un gouvernement ou un parti politique au pouvoir.

Les caisses populaires auraient dû disparaître: elles ont été créées par et pour les pauvres et nous ne sommes plus pauvres. Elles ont été créées pour encourager l'épargne. Elles avalent une motivation nationaliste et le nationalisme nous divise. Pourtant les caisses sont plus florissantes que jamais. C'est sans doute qu'on leur a trouvé un autre usage. (37)

34- Idem, P. 14

35- Idem, p. 14 .

36- Idem, p. 14 .

37- Les assises en résumé $\left(14^{\mathrm{e}}\right.$ congrès des dirigeants et dirigeantes de Desjardins), Lévis, C.C.P.E.D.Q. Déc. 1986, p.4 
C'est dans cette adaptation aux besoins du milieu que la caisse populaire trouve sa nécessité. "... par un souci communautaire du bonheur, par une volonté de vivre la démocratie, par une acceptation loyale des droits humains》(38), la caisse populaire Saint-Jean-Eudes, plus tard le point de services, a su répondre aux attentes de la collectivité par laquelle et pour laquelle elle a été fondée. Corriger les quelques faiblesses qui la caractérisent lui permettrait de renouveler ses formes sociales d'appartenance.

\subsection{LE SYSTEME PERTINENT.}

3.3.1 Définition du système pertinent.

Les services financiers du Point de services sont un ensemble d'activités ayant pour objectif de permettre aux dirigeants, cadres et employés de la caisse populaire d'Arvida d'offrir aux sociétaires de Saint-Jean-Eudes toute la gamme des services qu'ils sont en droit d'obtenir d'une institution financière. A ceux déjà existants soient: les dépôts, les retraits et la perception de comptes divers, s'ajouteraient les services spécialisés d'épargne et de crédit (les différentes formes de placement à long terme et les différentes sortes de prêts disponibles). Ce système devrait permettre à la Caisse populaire d'Arvida de récupérer une large partie de la clientèle perdue depuis 1'abolition, en 1980, des services spécialisés d'épargne et de crédit. Elle pourrait peut-être également accroître sa part de marché.

38- Idem, p. 5 
3.3.2 Validation de la définition.

- Propriétaires: Les propriétaires du système sont la Caisse populaire d'Arvida représentée par le conseil d'administration et le directeur.

- Environnement: La population du secteur Sant-Jean-Eudes de la ville de Jonquière pour laquelle le Point de services représente la seule institution financière du milieu immédiat.

Des individus de Chicoutimi et des environs qui empruntent le boulevard du Saguenay et qui arrêtent au Point de services au retour du travail.

- Clients:

Le conseil d'administration de la Caisse, le directeur et ses deux adjoints.

- Transformation: Instaurer des services financiers de façon à ce que les sociétaires de Saint-JeanEudes puissent bénéficier de toute la gamme des services spécialisés d'épargne et de crédit, sur place, dans les locaux du Point de services. 
- Acteurs:

- Point de vue:
Le directeur de 1a Caisse, I'adjoint courant et administratif et I'adjoint conseil qui auront à opérationnaliser les transformations proposées.

En implantant des services spécialisés d'épargne et de crédit, on redonne au milieu de Saint-Jean-Eudes, particulièrement aux membres de 1a Caisse, tous les services financiers que sa population s'était donnés avant 1980.

Par ces activités on espère raviver et ramener la clientèle qui avait opté pour une autre institution financière depuis la disparition de ces services en 1980.

L'addition des nouveaux services permet aux administrateurs d'anticiper une croissance du chiffre d'affaires à Saint-Jean-Eudes .

II est aussi permis d'espérer que, avec ces nouveaux services, le Point de services atteigne un niveau de pénétration comparable à celui de $I^{\prime}$ ensemble des caisses populaires du Saguenay-Lac-Saint-Jean, soit 
$56,9 \%$ en 1985 . Actuellement, Ies renseignements que nous possédons donnent un taux de pénétration inférier à $50 \%$ à Saint-JeanEudes (1092 comptes pour une population de près de 2 279).

- REMARQUE:

L'auteur de cette étude étant un sociétaire de la Caisse populaire d'Arvida et un membre de son conseil d'administration, il devient propriétaire et client du système proposé.

CONCLUSION

Les nombreuses lectures qui ont précédé la rédaction du $3^{e}$ chapitre ont amené I'auteur à discourir sur l'importance socio-économique des caisses populaires Desjardins au Saguenay-Lac-Saint- Jean. Implanté dans notre région depuis plus de 75 ans, le système coopératif Desjardins a su démontrer la nécessité de sa présence au sein des communautés où une coopérative d'épargne et de crédit s'était établie. La caisse populaire a toujours su adapter ses ressources financières et humaines aux besoins des collectivités petites ou grandes. Sa présence grandissante dans une forte proportion de municipalités de la région, justifie son maintien au service de la population qui l'a constituée, I'a alimentée, l'a dirigée et lui a permis de devenir ce qu'elle est. 
C'est dans cet esprit que les cadres de la Caisse populaire d'Arvida, de même que les membres du conseil d'aministration ont admis l'importance et la valeur du Point de services. Il restait à imaginer quelques façons d'y améliorer la qualité des services offerts. Le système pertinent proposé répond à ce besoin d'amélioration; il élimine les inconvénients liés aux espaces physiques intérieurs et extérieurs en raison des changements que son application impose.

Les chapitres suivants diront de quelle façon il serait possible d'opérationnaliser la nouvelle gamme de services. Ils élaboreront également sur les changements que le système proposé demande. 
CHAPITRE IV

CONCEPTUALISATION DE LA SOLUTION RETENUE 


\section{CHAPITRE IV}

\section{CONCEPTUALISATION DE LA SOLUTION RETENUE}

\section{INTRODUCTION}

Au chapître précédent nous avons pu établir qu'il devenait important de rétablir les services spécialisés d'épargne et de crédit au Point de services. En effet, l'étude sur le rôle socio-économique régional du mouvement des caisses populaires Desjardins a clairement mis en relief cette nécessité pour une collectivité restreinte et quelque peu isolée, de se doter de services financiers qui lui appartiennent et qui, par surcroît, répondent à l'ensemble de ses besoins.

Soumise à la direction de la Caisse, la solution proposée au chapître précédent a vite reçu I'assentiment des personnes concernées. Au conseil d'administration, un certain consensus est à s'établir sous l'influence des quelques administrateurs provenant de Saint-Jean-Eudes.

Le présent chapître vise donc à développer une conception visuelle de la solution préconisée. Pour ce faire il faudra rassembler un minimum d'activités interreliées par les flux appropriés. Ce processus vise simplement à rendre fonctionnelle la solution proposée au sujet de 1'amélioration des services financiers.

Puis après avoir validé le modèle selon les critères établis par Peter B. Checkland, il sera possible de le comparer avec la réalité. Cette activité n'a d'autre raison d'être que celle d'une identification des changements à réaliser pour rendre le modèle opérable et opérant dans les locaux du Point de services. 


\subsection{LE MODELE CONCEPTUEL}

Le modèle systémique suggéré ne peut se comprendre que s'il est exposé sur deux niveaux d'action. Le système de base identifie d'abord les fondements généraux des activités essentielles au fonctionnement d'une institution de type bancaire ou caisse populaire. Quant au deuxième niveau d'action, il décrit les activités des 4 sous-systèmes permettant au système de base de bien s'articuler autour des différentes personnes qui auront à l'opérer et de bien répondre aux besoins des utilisateurs du Point de services.

4.1.1 Système des opérations du Point de services.

Tel que présenté, le système général des opérations du Point de services se divise en 5 sous-systèmes interdépendants. Ce sont:

- Les préparatifs d'ouverture du Point de services.

- Les transactions courantes.

- La négociation des placements et des prêts.

- La réception du numéraire.

- Les opérations de fermeture du Point de services.

le sous-système des opérations de fermeture du Point de services, ayant peu ou pas d'influence sur le type, la quantité et la qualité des services offerts, il a été intégré et décrit dans le système général. 
Point de services.

La figure IX qui suit trace le schéma du système de base du SYSTEME DES OPERATIONS DU POINT DE SERVICES

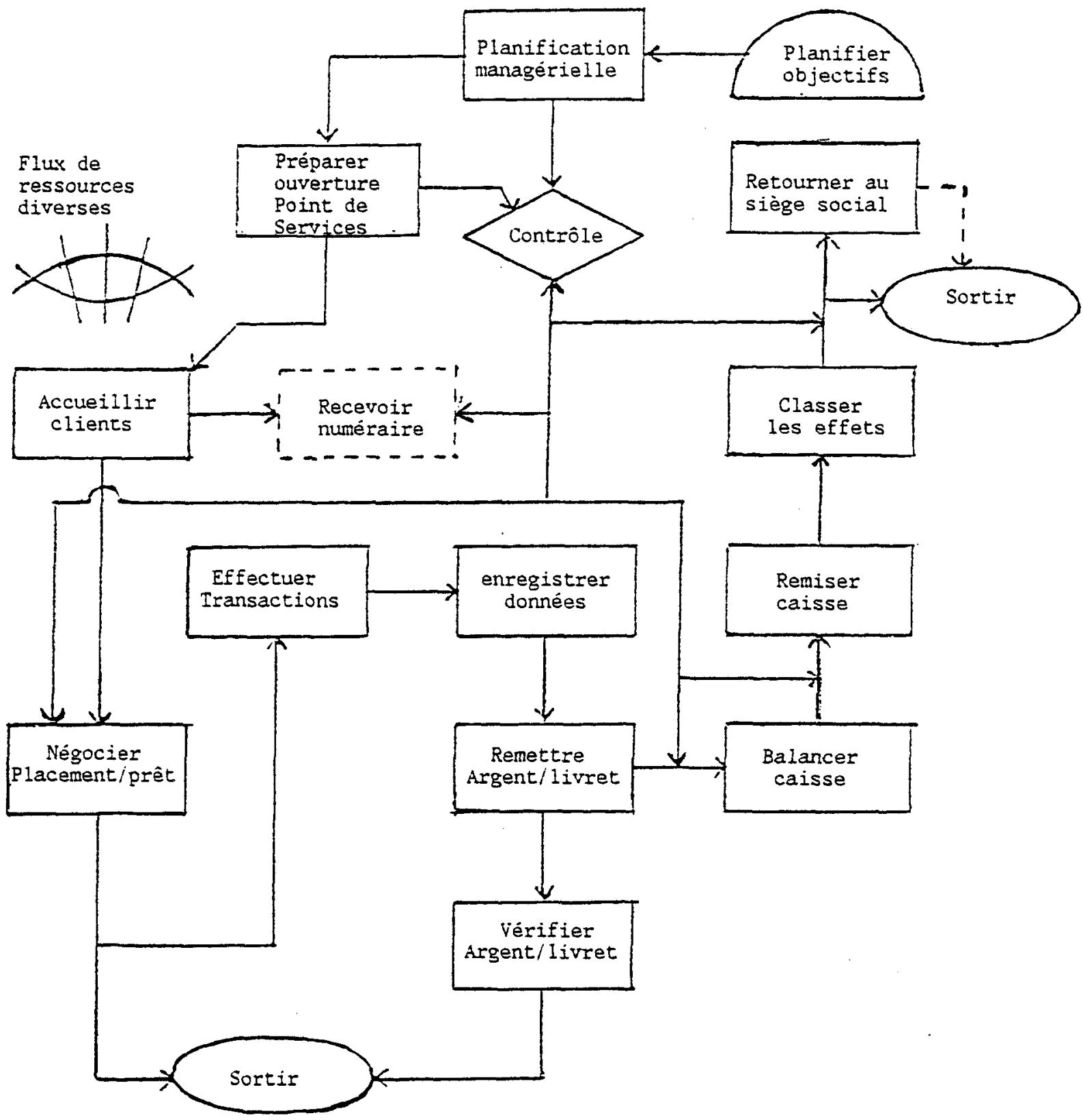

FIGURE IX 
4.1.2 Les sous-systèmes d'activités.

4.1.2.1 Les préparatifs d'ouverture du Point de services.

La description de ce sous-système fournit les renseignements sur la façon de démarrer les opérations au Point de services. Quelques constatations majeures sont à retenir. D'abord un seul des employés du Point de services doit passer au siège social de la Caisse pour y recueillir les directives et effets nécessaires au fonctionnement du Point de services. Le fait que cet employé doive distribuer aux caissières la répartition de leur travail lui confère une certaine autorité sur le fonctionnement du Point de services. Enfin, le diagramme du sous-système nous indique que les caissières et l'autre employé (qu'on pourrait appeler Assistant-conseil et administratif) auraient à accueillir les clients à deux niveaux différents. Les caissières seraient affectées à l'accueil des clients qui viennent pour effectuer les transactions courantes. L'assistant-conseil et administratif aurait la responsabilité de contrôler la réception du numéraire et d'accueillir les sociétaires désireux de négocier un prêt ou un placement. On peut visualiser tout cela à la figure X qui suit. 
SOLS-SYSTEME: PREPARER OUVERTURE DU POINT DE SERVICES

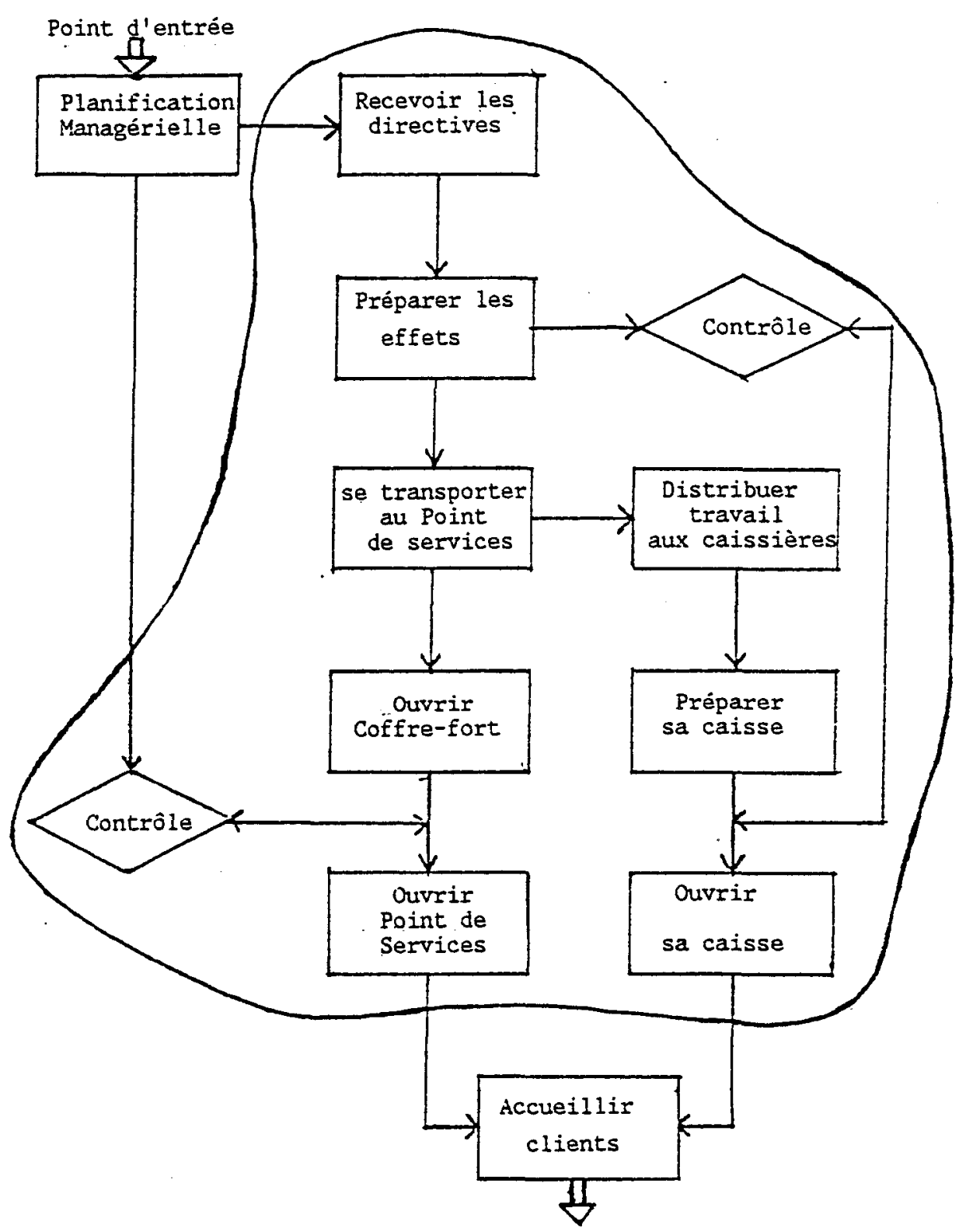




\subsubsection{Les transactions courantes}

Ce sous-systène comprend les 3 opérations courantes effectuées par les sociétaires, les dépôts, les retraits, les paiements des comptes acceptés. Les opérations sont effectuées par les caissières. La figure XI qui suit nous explique le fonctionnement de ce sous-système.

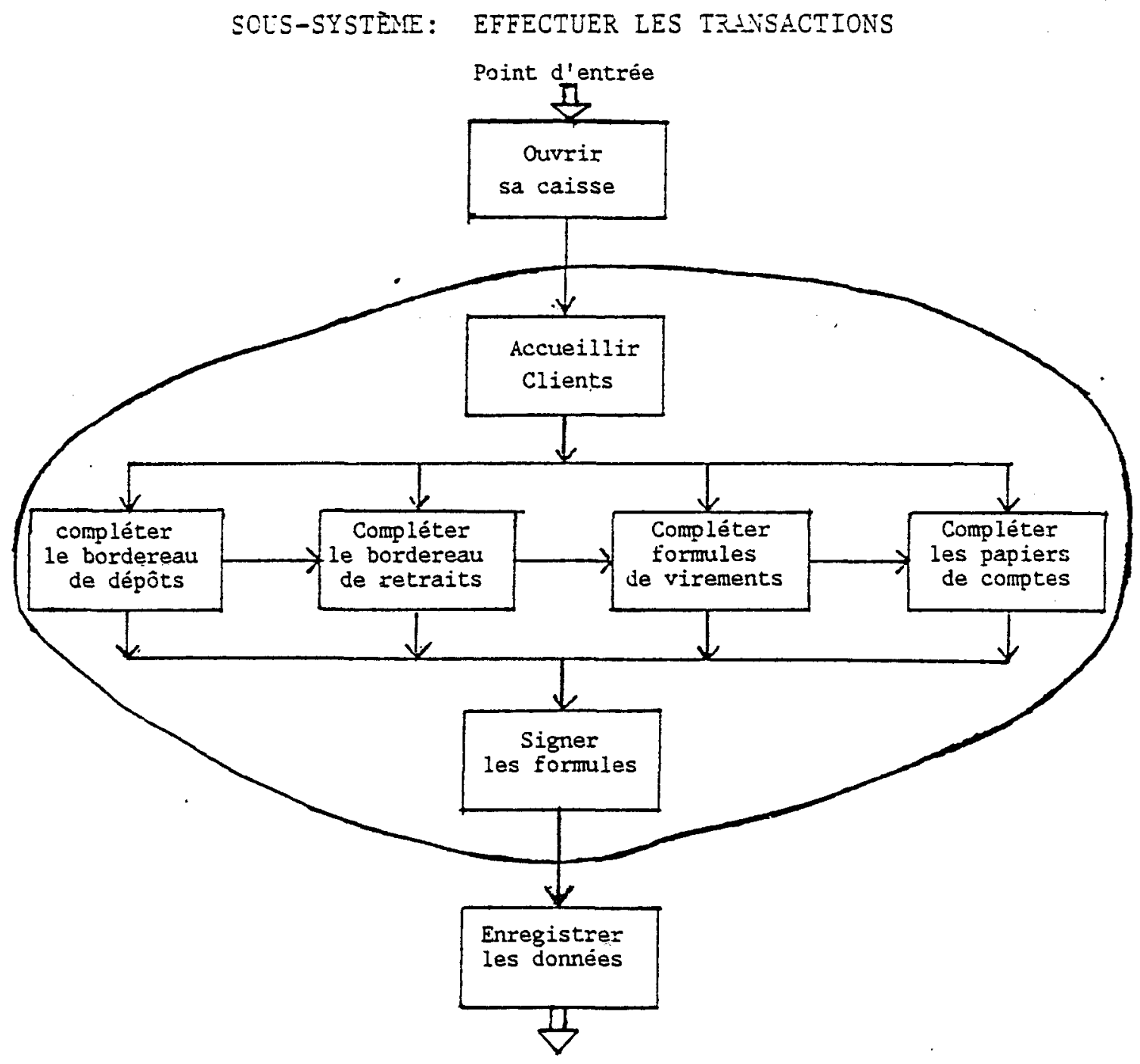

FIGURE XI 
4.1.2.3 La négociation des placements et des prêts.

Le sous-système constitue la nouveauté à implanter au Point de services. Ce nouveau service serait sous la responsabilité d'un officier de la Caisse spécialement formé pour ce genre de transactions. La figure XII qui suit décrit les activités qu'il faut accomplir à ce niveau.

SOUS-SYSTEME: NEGOCIER PLACEMENT/PRET

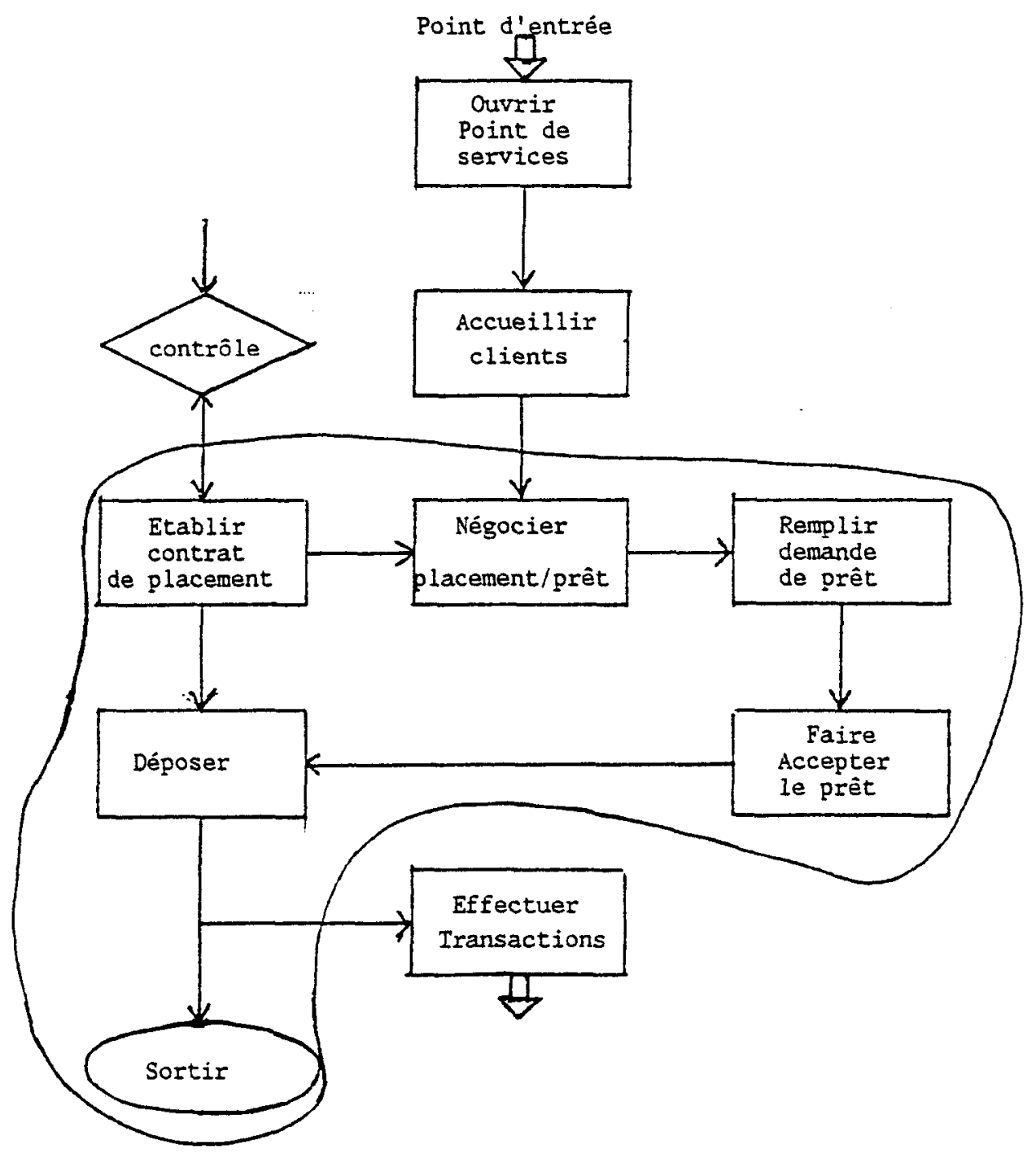

FIGURE XII 


\subsubsection{La réception du numéraire}

De par I'organisation systémique décrite dans les quelques pages qui précèdent, l'officier préposé au placement et au prêt devient Ie coordonnateur des activités de Point de services. Pour cette raison, il devient aussi le responsable du processus de réception du numéraire. La figure XIII reproduit le schéma du fonctionnement de cette activité.

SOUS-SYSTEME: RECEVOIR LE NUMERAIRE

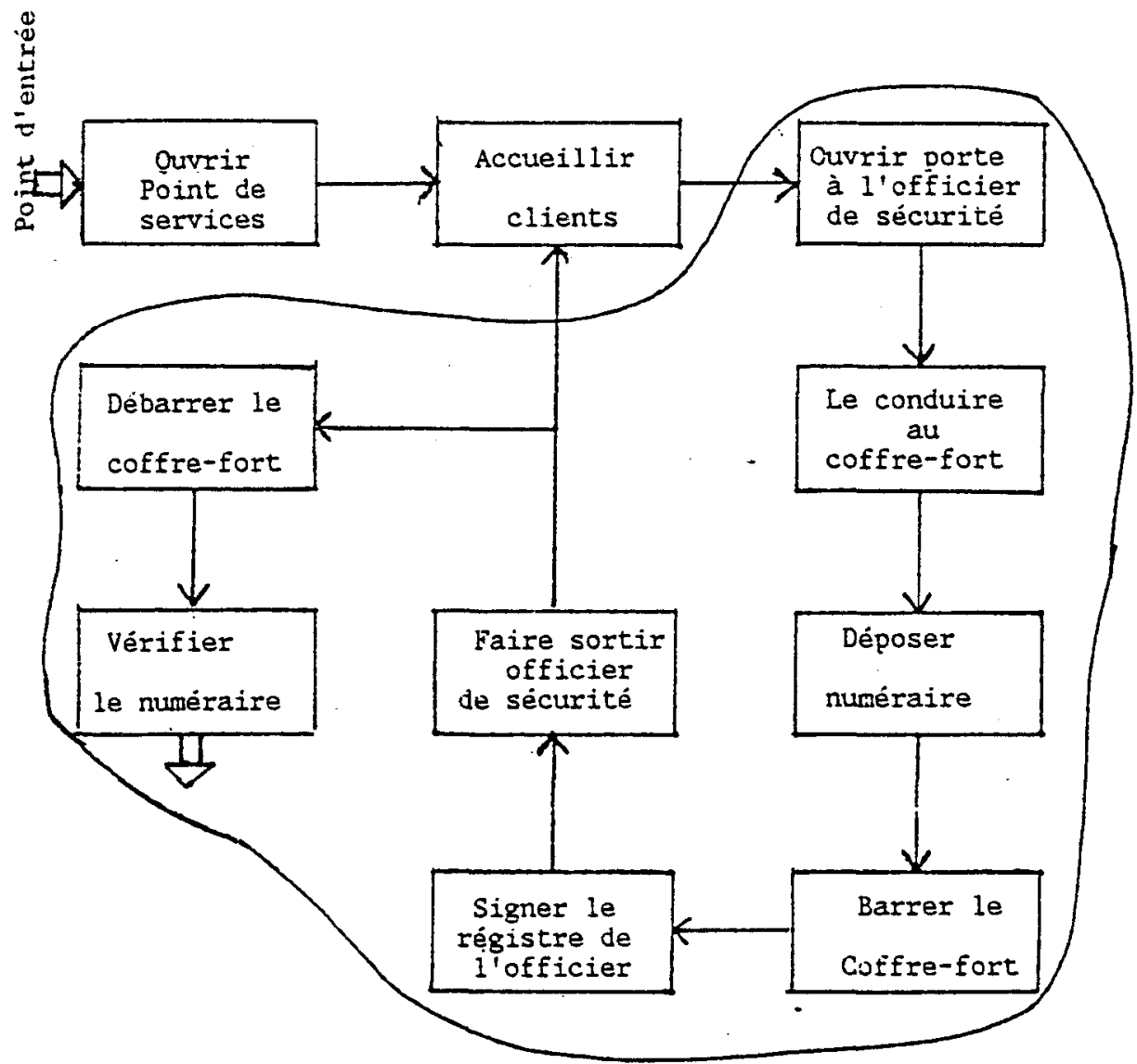


4.2 VALIDATION DU MODELE CONCEPTUEL

Dans un document intitulé: L'organisation, un système d'activités humaines: Conception d'un modèle général, Paul Prévost identifie 8 caractéristiques qu'on peut utiliser pour valider n'importe quel modèle conceptuel de système d'activités humaines. Ce sont:

1) Le système a un objectif, une mission, une fonction.

2) Le système a des mesures de performance et des mécanismes de contrôle.

3) Le système a des composantes ellesmêmes systèmes avec toutes les caractéristiques appropriées.

4) Les composantes du système ont un certain degré d'interrelation permettant à un effet de se permuter dans tout le système.

5) Le système est un système ouvert. I1 existe dans un système plus large ou/et à un environnement avec lequel il échange. Les frontières d'un système sont définies par les preneurs de décisions impliqués.

6) Le système a des ressources physiques et humaines.

7) Le système a des preneurs de décisons et un processus de décison.

8) Le système a une certaine garantie de continuité, il n'est pas éphémère et récupèrera après une crise.(1)

1- Prévost, Paul, L'organisation, un systme d'activités humaine: Conception d'un modèle général, Chicoutimi, LEER, 1980, p.14. 
C'est donc à partir de l'application de ces caractéristiques à notre modèle conceptuel que nous allons pouvoir vérifier la cohérence et la valeur du modèle proposé.

Le système proposé vise à atteindre l'objectif suivant: offrir aux sociétaires de Saint-Jean-Eudes une gamme de services bancaires de la meilleure qualité possible et les plus complets qui soient. Le système proposé, exigeant des modifications physiques des espaces se propose comme mission de simplifier la problématique des files d'attente, d'accrôtre le nombre de déposants.

Le système pourra toujours mesurer ses performances; le calcul et l'analyse du trop perçu demeure la mesure financière permètant aux cadres et dirigeants d'évaluer si la croissance du Point de services continue de maintenir une rentabilité nécessaire à son fonctionnement. D'autres mécanismes de contrôle assureront l'efficacité et la qualité des services offerts.

Le système de base se partage en 5 sous-systèmes ayant chacun les caractéristiques d'un système.

Les sous-systèmes sont interreliés les uns aux autres par des flux de monnaie et d'informations. Il sont également interdépendants; le sous-système des transactions ne saurait fonctionner sans le soussystème de réception du numéraire. Les trois sous-systèmes d'opérations financières sont encadrés par les opérations d'ouverture et de fermeture du Point de services. 
Le système est ouvert puisqu'il se situe dans l'ensemble du système des services bancaires. Il est à I'usage des sociétaires de Saint-Jean-Eudes, mais il offre ses services à toute la collectivité du secteur.

Le système requiert des ressources humaines, physiques et monétaires.

Le système comprend 3 niveaux décisionnels: l'employé responsable du Point de services, les cadres du Siège social et les membres du Conseil d'administration.

- La continuité et la permanence du système sont assurées car dans son essence même le système existe depuis plus de 30 ans. Les différentes variations dans l'organisation de ses sous-systèmes lui procure un élan nouveau pour enrayer la diminution du nombre de sociétaires, crise à laquelle le Point de services est confronté présentement.

\subsection{COMPARAISON AVEC LA REALITE.}

La situation actuelle, en ce qui a trait aux services bancaires offerts à Saint-Jean-Eudes, oblige la population de ce secteur à se déplacer sur plusieurs kilomètres pour négocier des emprunts ou des placements à long terme. A cause de cette démarche obligatoire, le futur emprunteur ou celui qui veut placer son argent sur de longues périodes se retrouve en plein coeur de la concurrence. Car au Carré Davis, la Banque Nationale, la Banque Royale, la Banque de Montréal, le Trust Royal, la 
caisse d'économie des employés de l'Alcan et la Caisse populaire d'Arvida offrent ces services financiers.

Le fait, pour la Caisse, de développer à son Point de services un système de services spécialisés d'épargne et de crédit crée dans le secteur de Saint-Jean-Eudes une situation d'exclusivité. Face à la collectivité du secteur, la Caisse met en place une structure financière de quasi-monopole. La Caisse peut donc espérer s'emparer de la presque totalité du marché des services spécialisés d'épargne et de crédit.

De la fin de l'année 1950 à la fin de I'année 1980, la population de Saint-Jean-Eudes s'était habituée à la présence dans le milieu de la totalité des services bancaires courants. Depuis 1980, le sous-système de négociation des prêts et placements a été centralisé au siège social de la Caisse sur le Boulevard Mellon à Jonquière. Ce fait a provoqué l'exode de plusieurs sociétaires de Saint-Jean-Eudes vers d'autres institutions financières. Le système proposé permet à la Caisse de corriger cette situation.

Lorsque nous nous présentons au Point de services, les jours d'affluence, nous assistons à une certaine congestion au niveau de I'entrée des clients. En effet, chaque guichet a sa propre file d'attente; et celle du guichet numéro l se prolonge jusque dans les portes d'entrée obstruant celles-ci. Le système proposé nécessite des changements importants dans I'aménagement physique des lieux. II sera donc possible, avec le nouveau système de corriger le problème des deux files d'attente en les fondant dans une seule. 
Lors des mêmes jours d'affluence, il se présente également certaines complications au niveau des stationnements. La seule façon pour le système proposé d'améliorer la situation et d'éliminer le bloquage du terrain de stationnement réside dans la rapidité du service qu'il serait possible de lui faire donner.

\section{CONCLUSION}

En résumé, la grande logique et la philosophie de base qui sous-tend le système proposé est de restituer à la population de Saint-Jean-Eudes, l'ensemble des services financiers qu'elle s'était elle-même donnés il y a plus de 35 ans.

Le système proposé n'est pas parmi les plus complexes à réaliser. Dans son schéma de base, il est fondé sur des données paramétriques connues de l'ensemble des entreprises de services financiers. L'important réside surtout dans les modifications à apporter dans les structures. Différentes modalités d'applications du système peuvent être envisagées:

Le chapitre suivant présentera donc comment il serait possible d'implanter le système proposé. Nous y présenterons les changements physiques, organisationnels et fonctionnels à apporter et nous essaierons d'établir des coûts possibles pour ces changements. 
CHAPITRE V

IDENTIFICATION DES CHANGEMENTS POSSIBLES 


\section{CHAPITRE V}

\section{IDENTIFICATION DES CHANGEMENTS POSSIBLES}

INTRODUCTION

Le chapître précédent nous a permis de prendre connaissance d'une structure systémique idéale à implanter au Point de services dans le but d'accroître et d'améliorer les services offerts à la clientèle. Le présent chapître nous invite donc à considérer des changements qu'il faudrait effectuer pour rendre le système opérationnel. Compte tenu du fait que le système proposé en est un de service très personnalisé, nous proposerons également quelque alternative différente soit dans les coûts, soit dans la personnalisation du service, soit dans la structure fonctionnelle.

Ainsi, dans la première partie du chapître nous analyserons les changements que devrait entraîner le modèle conceptuel proposé. Quant à la deuxième partie, elle élaborera sur quelque alternative possible.

Il est aussi une dimension qu'il ne faut pas oublier; soit qu'on ne peut déterminer de délai de temps pour l'implantation du modèle proposé.

Enfin, le modèle idéal proposé et toute alternative possible suggérée répondent toujours au même objectif qui consiste à fournir à la population de Saint-Jean-Eudes des services de meilleure qualité ainsi que des services plus complets qui incluent ceux du crédit et de 1 'épargne spécialisée à terme. 


\subsection{LES CHANGEMENTS DUS AU MODELE PROPOSE}

Pour être capable d'implanter le modèle proposé, des changements importants doivent être apportés à trois niveaux: I'aménagement des espaces physiques, 1'utilisation des ressources humaines et la structure décisionnelle et de fonctionnement.

\subsubsection{L'aménagement des espaces physiques.}

Pour abriter le Point de services, la Caisse populaire d'Arvida est en situation de location par bail avec Ville de Jonquière. Jusqu'à la fin mars 1986, la certitude d'un renouvellement automatique du bail annuel pour plusieurs années encore nous apparaissait plutôt une incertitude. Or, depuis ce temps, il semble que les plans de ville de Jonquière se soient quelque peu modifiés puisque, d'après les contacts du directeur de la Caisse avec la mairie, l'urgence d'une relocalisation du Point de services s'est considérablement atténuée au point d'être reportée sur plusieurs années.

Donc face à une amélioration de la quantité et de la qualité des services offerts au Point de services, il est loisible pour les administrateurs de placer en veilleuse ce projet de relocalisation du Point de services. Envisager un réaménagement des espaces physiques intérieurs nous paraît plus à propos. 


\subsubsection{Aménagement intérieur.}

Instaurer les services spécialisés d'épargne et de crédit oblige les dirigeants de la Caisse à rénover l'intérieur du Point de services afin d'aménager un bureau pour recevoir les sociétaires désireux de négocier un prêt d'argent ou un placement à long terme. La façon la plus facile d'y arriver consiste à modifier l'emplacement des quichets de services afin de libérer 1 'accès au bureau pratiquement inoccupé situé à I'arrière du comptoir blindé actuel.

En modifiant également la structure des portes d'entrée du Point de services, il est aussi possible de transformer les deux files d'attente actuelles en une seule plus longue moins incombrante et utilisant de façon plus adquate les espaces alloués aux clients.

A 1'intérieur des locaux du Point de services, trois guichets de services seraient accessibles. Les deux premiers fourniraient les opérations complètes de transaction en y incluant l'utilisation du terminal. Quant au troisième guichet, il deviendrait un guichet de service rapide ne nécessitant pas l'utilisation du terminal (échange de chèque, paiement de factures...). Ce dernier guichet n'entrerait en opération que lors dès jours d'affuence. Il pourrait être opéré par le responsable du crédit et des placements lorsque ce dernier n'aurait pas de clients à rencontrer.

Le plan des nouveaux aménagements des locaux présenté à la page suivante permet de visualiser tous ces changements. 
REAMENAGEMENT INTERIEUR DU POINT DE SERVICES

Echelle $1 / 4$ de pouces $=2$ pieds Ramené à $75 \%$

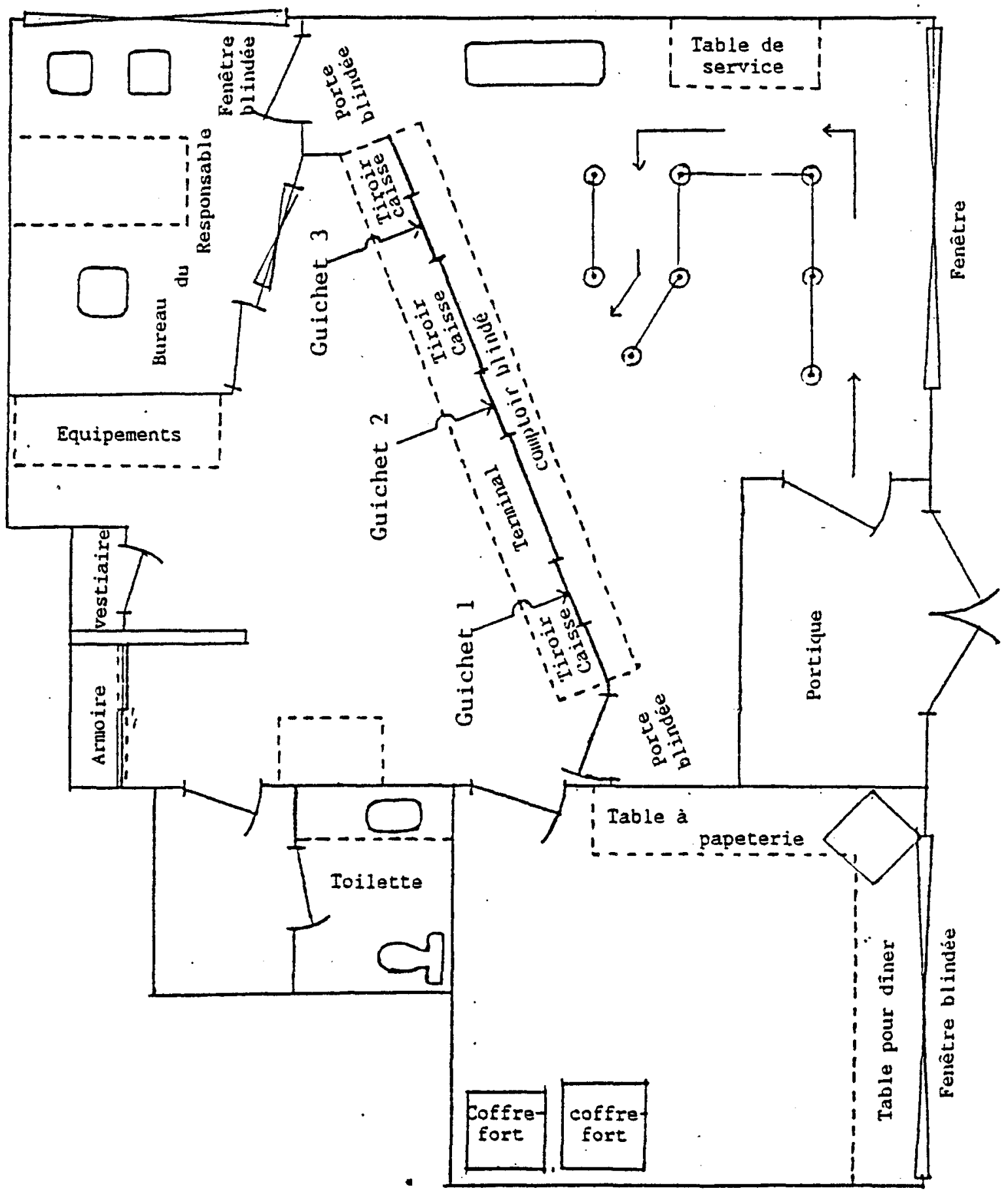

FIGURE XIV 


\subsubsection{Aménagement extérieur.}

Il serait souhaitable de modifier la partie avant des aires de stationnement. Mais comme la Caisse n'est pas propriétaire du terrain, il semble peu probable qu'on puisse obtenir des modifications. Cependant, advenant le cas d'une croissance appréciable du nombre de sociétaires, il y aurait lieu d'envisager la possibilité de louer et faire aménager quelques aires de stationnement chez le $2^{\mathrm{e}}$ voisin en direction de Chicoutimi.

Présentement il faut s'en tenir aux espaces actuels et axer plutôt les modifications vers une accélération des services pour enrayer les encombrements aux heures de grande affluence.

\subsubsection{Modifications aux ressources humaines.}

\subsubsection{Création d'un nouveau poste.}

Afin d'assurer les services spécialisés d'épargne et de crédit, il faudrait procéder à l'embauche d'un agent d'épargne et de crédit. À cette personne seraient conférés les tâches et pouvoirs suivants.

1) Se présenter au siège social de la Caisse à tous les jours, pour cueillir les directives le matin et faire rapport le soir.

2) Accomplir toutes les tâches relatives à l'ouverture et à la fermeture du Point de services.

3) Organiser et contrôler le travail de la (des) caissière(s). 
4) Etre responsable de la réception et de la vérification du numéraire.

5) Répondre aux demandes de prêts et de placements.

6) Donner le service à la caisse rapide lorsqu'il y aura affluence et pas de demandes aux services spécialisés d'épargne et de crédit.

7) Recevoir les sociétaires de Saint-Jean-Eudes qui ont un prêt hypothécaire ou un certificat de placement à renouveler.

8) S'occuper de la promotion du Point de services de façon à augmenter le volume de ses prêts et de ses placements.

Comme cet employé jouerait jusqu'à un certain point le rôle d'un assistant-gérant, le poste nouvellement ouvert en serait un un peu plus élevé. On pourrait facilement coiffer ce poste du titre de: assistant conseil et administratif. En tant qu'assistant conseil, cet officier dépendrait de l'adjoint conseil et coordonnerait les activités du crédit et des placements à termes au Point de services. En tant qu'assistant administratif, il serait sous l'autorité du directeuradjoint aux services courants et administratif. Il coordonnerait les opérations courantes du Point de services. La(les) caissière(s) serait (ent) sous sa responsabilité. 


\subsubsection{Le travail de caissière.}

Le travail de caissière se trouve très peu modifié par 1'arrivée des nouveaux services. Le service au guichet continue d'être le même. Mais la caissière, n'a plus à se rendre au Siège social pour recevoir les directives; elle ne s'occupe plus de la réception du numéraire et elle n'effectue plus les opérations d'ouverture et de fermeture de Point de services.

\subsubsection{Modifications de nature fonctionnelle et décisionnelle.}

\subsubsection{Modification de nature fonctionnelle.}

Dans le but d'accélérer le service aux heures de grande affluence, il y aurait lieu de tenter l'expérience d'une caisse rapide opérée, dans la mesure de sa disponibilité, par l'assistant-conseil et administratif. Cette caisse n'aurait d'autre rôle que celui de recevoir les clients qui ne veulent qu'échanger des chèques, payer des comptes sans avoir à utiliser leur carnet de caisse et le terminal.

\subsubsection{Modification à la structure décisionnelle.}

En créant un poste de responsabilité comme celui d'assistantconseil et administratif, il devient nécessaire de réorganiser la structure administrative et décisionnelle entourant le fonctionnement du Point de services. La structure actuelle, comme le révèle l'organigramme de la figure II au chapître II, fait dépendre toutes les employées du Point de services directement de I'adjoint-courant et administratif. 
Avec le nouveau poste d'assistant consell et administratif, la structure hiérarchique décisionnelle se trouverait modifiée de la façon illustrée par I'organigrame de la figure XV qui suit.

ORGANIGRAMME DU POINT DE SERVICES

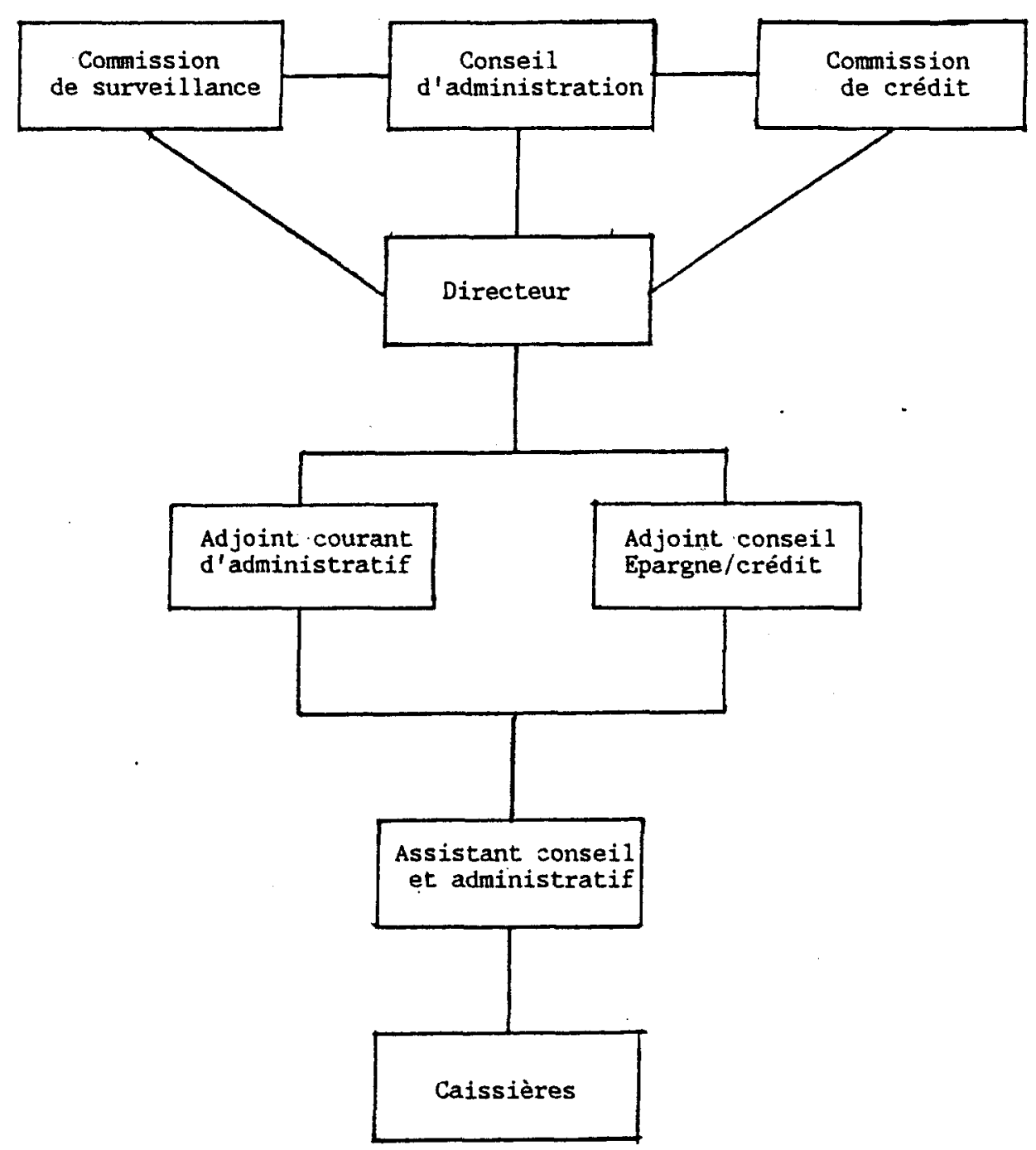




\subsubsection{Coûts approximatifs des changements}

Les dépenses à encourir pour instaurer les services spécialisés d'épargne et de crédit au Point de services sont de deux ordres. D'abord il $\mathrm{y}$ a les coûts des aménagements intérieurs des locaux. A ceux-ci s'ajoutent les coûts d'embauche de personnel supplémentaire.

5.1.4.1 Coûts des rénovations

1) Réfection du portique

$2500 \$$

2) Réaménagement du comptoir

$3 \cdot 000$

3) Aménagement du bureau de

I'assistant conseil et administratif

3000

4) Ameublement décoration

climatisation, divers

1500

Coût total des rénovations

$10000 \$$

\subsubsection{Coûts de l'embauche de personnel}

Actuellement à la Caisse populaire d'Arvida, un agent d'épargne et de crédit traite environ 560 dossiers de prêts de tous genres. A cette tâche s'ajoutent les placements à terme. Il y avait au Point de services, à la fin de 1985, 310 dossiers de crédit totalisant plus de 2 milllions de dollars. En plus on comptait pour près de un million de dollars en épargne à terme. Donc, au siège social, une demi-tâche d'agent d'épargne et de crédit est consacrée au traitement de dossiers provenant du secteur de Saint-Jean-Eudes. Si on transfert un agent d'épargne et de crédit au Point de services et qu'on le nomme responsable 
du Point avec quelques tâches additionnelles, il en coûtera une demitâches d'agent d'épargne et de crédit en plus à la Caisse. A ce salaire, il faudrait ajouter un boni pour les responsabilités suplémentaires; ce qui représente une somme maximale de 15000 \$, en y incluant les bénéfices marginaux.

Globalement, il en coûtera environ 25000 \$à la Caisse, pour rendre opérationnelle la solution proposée. Mais si on amortit sur 5 ans les réparations à l'immeuble et qu'on inclut un manque à gagner de $1000 \$$, il s'en suit que la dépense réelle financière est de 18000 \$a première année.

\subsection{Une solution alternative.}

Le modèle conceptuel présenté au chapître IV et qui nécessite les changements décris dans la partie 5.1 du présent chapitre constitue le modèle idéal de la structure de services à offrir à la population de Saint-Jean-Eudes. Il est idéal parce que très personnalisé. Il est idéal aussi parce qu'il redonne au secteur un ensemble de services que sa population avait mis plus de 25 pour s'en doter. Enfin, il est idéal parce qu'il met en place des structures et du personnel en vue d'une croissance du Point de services.

Une autre alternative de solution et de changements a également été envisagée. Comme la solution, elle garde en priorité l'objectif de fournir à la population de Saint-Jean-Eudes les services spécialisés d'épargne et de crédit directement au Point de services. 
A titre informatif nous vous présentons cette solution de rechange.

Dans cette solution, nous utilisons sensiblement le même modèle conceptuel que celui du projet priorisé. La principale différence se situe au niveau du processus d'ouverture du Point de services et de l'accueil des clients. Cette variante du modèle idéal permet à la Caisse d'envoyer un agent d'épargne et de crédit à Saint-Jean-Eudes seulement deux jours et demi (2 1/2) par semaine répartis comme suit: Les lundi et jeudi durant toute la journée et le vendredi en après-midi.

I1 va sans dire que de cette façon, 1'agent d'épargne et de crédit ne devient pas le responsable du Point de services. Tout comme dans la situation actuelle, il revient à la première caissière de procéder aux opérations d'ouverture du Point de services et de contrôler I'arrivée du numéraire.

Le réaménagement physique de l'intérieur des locaux demeure quand même obligatoire. Il est identique à celui du projet priorisé. Les coûts n'en sont pas modifiés.

La figure suivante redessine le processus d'ouverture du Point de services et $I^{\prime}$ accueil des clients. 
PROCESSUS D OUVERTURE DU POINT DE SERVICES

SOLUTION ALTERNATIVE

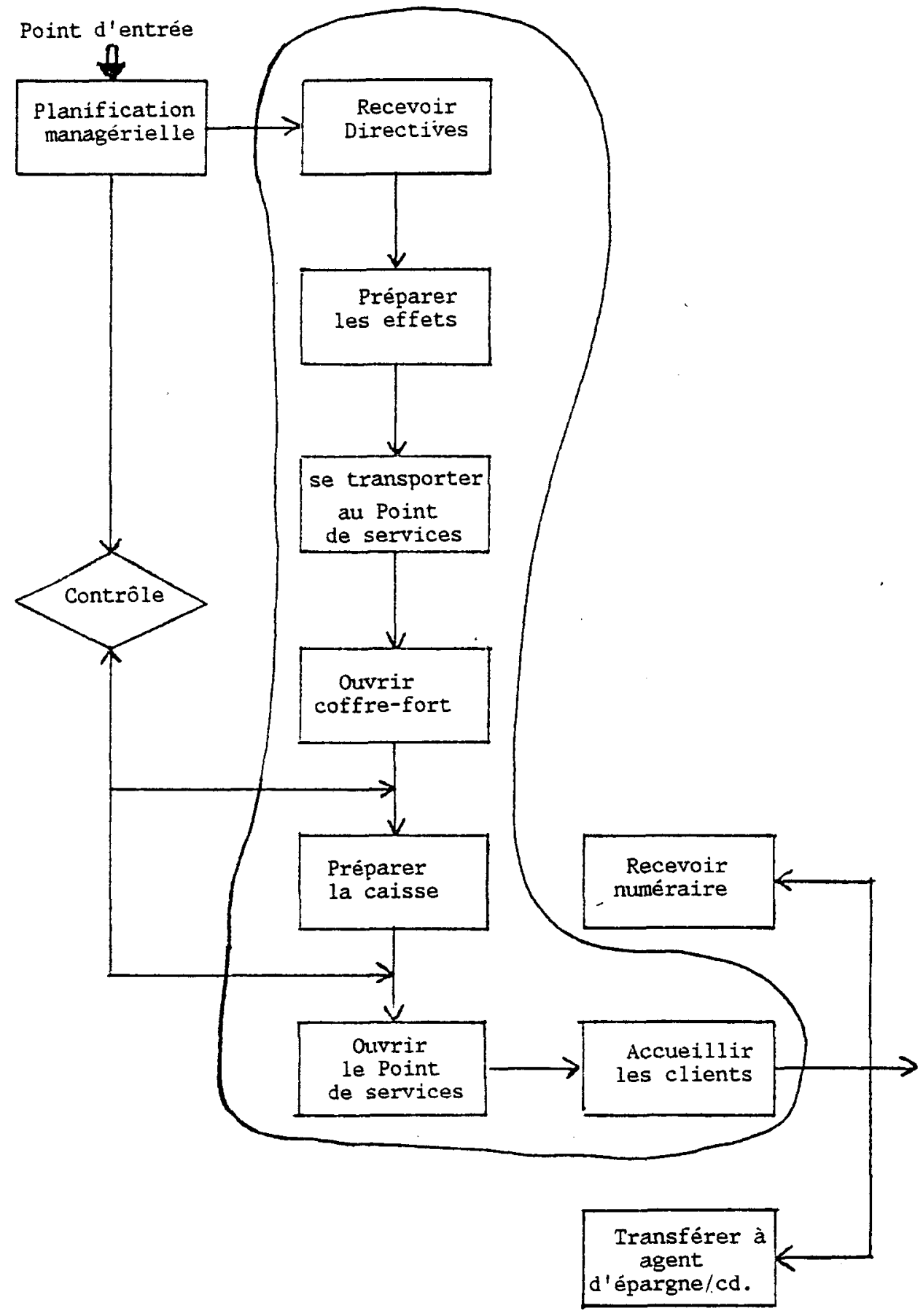


La seule économie à réaliser par le choix de cette alternative par rapport au modèle priorisé se situe au niveau salarial. Dans ce cas, en effet, la Caisse n'encourt aucun déboursé salarial parce qu'elle ne fait que procéder à un transfert de personnel.

A court terme, c'est-à-dire cette année, si le projet se concrétisait, cette alternative serait réalisable. Mais il faut plutôt envisager une solution à plus long terme qui devrait aller dans le sens d'une croissance du Point de services. C'est pour cette dernière raison que la solution alternative n'est pas le choix logique à retenir.

La solution alternative donnerait au Point de services des possibilités stagnantes de prêts et de placements à termes. Quand viendrait le temps de développer de nouveaux marchés, d'accroître le volume de prêts et de placements, il faudrait alors ajouter le personnel adéquat, lequel est déjà prévu et calculé dans le modèle idéal préconisé. Alors pourquoi ne pas privilégier un projet à long terme?

\section{CONCLUSION}

En raison des rénovations présentement en cours au siège social de la Caisse sur le Boulevard Mellon, il est impensable pour le moment, d'envisager des changements dans un avenir très court pour le Point de services. Il fallait donc orienter une recherche de solution à implanter dans un avenir plus lointain que l'intérieur d'une année. Le plan retenu prend ainsi une plus grande importance et offre, au delà de la situation actuelle, la meilleure façon de développer davantage le Point de services. 
CHAPITRE VI

REFLEXION METHODOLOGIQUE 


\section{CHAPITRE VI}

\section{REFLEXION METHODOLOGIQUE}

\section{INTRODUCTION}

Dans un document sur le diagnostic organisationnel, Paul Prévost écrivait "qu'une recherche-action réussie devrait apporter aux membres de l'organisation étudiée, une nouvelle compréhension de leur organisation, de ses comportements et de différents aspects des situations problématiques étudiées et la capacité d'appliquer ces nouvelles connaissances."(1). Quand on arrive au terme d'un tel genre de recherche, c'est sans doute l'objectif qu'on espère avoir atteint; du moins ce fut notre cas. Alors, quoi de mieux que de faire un retour sur notre méthode de travail afin de vérifier si elle nous a conduit à cette réussite.

\subsection{LA METHODE.}

Une recherche-action est un processus d'apprentissage qui vise l'autonomie des intervenants en leur permettant de sélectionner les situations prioritaires à corriger et d'y enclencher des mécanismes de solution. En ce sens, la méthodologie de Checkland oblige le chercheur à rechercher et diffuser un de ses fondements de base: la vision des choses.

1- Prévost Paul, Le diagnostic-intervention: Une approche systémique au diagnostic organisationnel et à la recherche-action, Chicoutimi, LEER, 1983, p.78. 


\begin{abstract}
Un système d'activités humaines comporte des activités interreliées par des flux variés, divers. Pour bien comprendre les réactions, les actions du système global, il fallait absolument en connaître celles des sous-systèmes qui le composent. Le diagnostic-intervention tel que nous l'avons pratiqué pendant cette recherche a permis de filmer toutes les parties du système à étudier.
\end{abstract}

En fait, on peut comparer l'application de cette méthode à la production d'un film ou d'une émission de télévision. En effet, dans la production d'un film, il a d'abord fallu que chacune des équipes de tournage mettent en place ses éléments de production: acteurs, éclairagistes, costumiers, I'organisation musicale,.... Chacune de ces équipes devait être fin prête. Une fois toutes les choses au bon endrolt, le jeu des acteurs a pu être fixé sur la pellicule. Et ainsi on a réussi à produire une première et on a noté toutes les faiblesses qu'on pouvait $\mathrm{y}$ trouver. Une fois ce travail fait, on a choisi des scénarios de correction qu'on a mis en application. On a fait cela jusqu'à ce qu'on soit capable de produire une version qui soit plus que satisfaisante pour tous les intervenants dans le film.

Quand on décortique cette description de la production d'un film et qu'on la compare avec notre recherche, il est possible d'établir un parallèle en trois parties.

\title{
- Première partie
}

La production de la première version du film avec toutes les activités qui la précèdent, c'est la même chose que construire l'image 
riche dans notre recherche. On a tracé le scénario le plus fidèle possible de la situation actuelle de l'entreprise étudiée. Chacune des composantes de l'entreprise et son environnement ont été décrits. C'est l'établissement de la problématique.

\section{- Deuxième partie}

L'analyse de cette première version du film a permis de déceler un certain nombre d'erreurs importantes à corriger. On a même décidé de celles qu'on allait corriger. Dans notre recherche, cette partie s'appelle le choix du système pertinent, de l'ancrage, c'est-à-dire choisir les situations les plus problématiques et définir comment elles seront solutionnées. A Saint-Jean-Eudes, on a déterminé que la problématique a retenir était celle de production de services à la clientèle et on a défini un système susceptible de corriger le système déjà existant.

\section{- Troisième partie}

Dans un film, la troisième étape consiste à refaire une version de la production à partir des scénarios de correction définis. On a donc refait le modèle pour qu'il soit à l'entière satisfaction des intervenants. Dans notre recherche-action, cette troisième phase s'appelait la conceptualisation, l'implantation et le contrôle. Pour le Point de services, le modèle conceptuel reprenait le système des services à la clientèle pour en reproduire un plus adéquat et plus complet. Il avait également été établi qu'il n'y aurait pas d'implantation immédiate du modèle proposé. 
Pour être capable d'en arriver à une production satisfaisante pour l'ensemble des intervenants, la méthodologie utilisée se devait de répondre à certaines normes de qualité.

\subsection{1 les qualités de la méthode.}

La méthode utilisée se devait absolument d'avoir une souplesse permettant au moins deux actions: 1) Pouvoir consulter le plus grand nombre d'intervenants du système.

2) Pouvoir modifier ou adapter notre rapport en fonction de l'évolution plus ou moins rapide du système dans le temps.

Avec le Point de services, nous avons vécu ces deux moments. Pour construire notre image riche, nous avons consulté des sociétaires du milieu, les employés, les cadres et quelques membres du C.A. De plus, nous avons dû évoluer et modifier notre problématique car, en cours de recherche, une situation qui allait devenir un élément de base de notre solution s'est transformée. Ville de Jonquière, qui ne devait plus renouveler le bail de location à la fin mai 1986, est revenue sur sa décision et acceptait de relouer une partie de son édifice à la Caisse avec une possibilité de plusieurs années encore. Donc notre idée de relocalisation du Point de services qui aurait chapeauté notre modèle conceptuel, il a fallu s'en départir, celle-ci devenant inutile.

Une deuxième qualité essentielle dans une méthode de diagnostic-intervention était que celle-ci soit contraignante. En effet, la méthode utilisée a obligé le chercheur à sélectionner avec beaucoup de 
rigueur intellectuelle et pratique, quels étaient les renseignements qu'il fallait retenir et ceux qu'il fallait rejeter. Elle obligeait également l'auteur à concilier les interventions diverses de façon à ce que le point de vue ou le fait relaté soit conforme à l'opinion de chacun de ces intervenants. Il faliait dégager des consensus.

Troisièmement, notre méthode de travail se devait d'être non limitative dans le temps ou les faits. Ce qu'il faut comprendre ici, c'est que, pour faire l'étude d'un système d'activités humaines, il est primordial que le système ne soit pas encadré par la méthode, mais plutôt que le système amène la méthode à suivre son évolution dans le temps tout en ne se privant d'aucun fait important; tout cela pour permettre de s'ajuster aux changements subits.

6.1.2 Les risques et dangers de la méthode.

Le principal risque ou danger de cette méthode provient de sa qualité d'être non limitative dans le temps. En effet, à cause de cela, à trop vouloir suivre l'évolution du système dans le temps, on court le risque d'être trop longtemps en phase de construction de l'image riche du système à étudier. Ceci pourrait entraîner une solution précipitée ne correspondant peut-être pas à toutes les attentes des décideurs. II importe donc à l'encontre de cet inconvénient, que l'auteur en accord avec les divers intervenants, s'établisse une limite dans les faits et le temps par un échéancier précis de son travail.

Un autre danger guette l'auteur si celui-ci est à la fois chercheur et décideur. Dans une situation semblable, le chercheur peut 
être tenté (ou tout simplement le faire sans trop s'en rendre compte) de négliger de consulter les autres décideurs, étant donné que c'est lui, comme chercheur, qui coordonne les activités d'étude et qui tresse les ficelles formant la toile de fond de la solution à prévoir.

\subsection{LA DEMARCHE}

Dans notre démarche, face à la problématique du Point de services, le deuxième danger souligné dans la partie précédente est devenu en quelque sorte notre premier piège. En effet, comme auteur de cette recherche-action, nous avons quelque fois été confronté avec notre deuxième rôle, celui de décideur, parce que nous étions membre du C.A. de la Caisse. Il était donc relativement facilè de s'approprier. comme décideur, le privilège d'être un des quelques administrateurs consultés. Fort heureusement, les consultations avec le directeur de la Caisse ont été plus que fréquentes. Comme c'est ce dernier qui, en réalité, pilote au C.A. Ia plupart des projets qui touchent le fonctionnement interne de I'entreprise, ses compétences financières et sa capacité de saisir et d'analyser une problématique, ont largement compensé pour la faiblesse précitée.

Il est aussi des facteurs extérieurs à la méthode qui peuvent influer sur la démarche. Nous avons vécu cette situation lorsque la maladie et la mortalité ont perturbé considérablement notre travail de recherche. Grâce à la non-complexité de la méthode et à ses qualités identifiées précédemment, le rattrapage a pu s'effectuer promptement et sans difficulté. Malgré tout, des ajustements ont dû être apportés parce 
que l'échéancier ne coincidait plus. Certains délais ont été consentis non par l'entreprise mais par I'Université elle-même. Pour la Caisse populaire d'Arvida il n'y avait pas urgence et cette absence de contrainte a contribué à diminuer cette pression qui pousse souvent 1'auteur d'une recherche à précipiter sa recherche de solution, rendant celle-ci moins appropriée peut-être.

L'élément de la démarche qui nous semble le plus positif ne se comprend pas, ne se mémorise pas, n'est pas abstrait; il se vit presque quotidiennement dans les contacts nécessaires que nous devons avoir avec les intervenants. Ces échanges sont sources d'enrichissement personnel par les attitudes d'écoute attentive qu'ils développent. Ils forment aussi une garantie d'efficacité de la méthode de diagnostic-intervention en recherche-action par les liens d'amitiés qu'ils créent souvent, mais surtout par la collaboration étroite qu'ils obligent.

\subsection{LES CONNAISSANCES ACQUISES.}

La plus grande qualité qui se dégage de la méthode adoptée pour l'ouvrage qui se termine, c'est la rigueur intellectuelle qu'elle génère dans notre esprit. Utiliser cette méthode une fois, c'est la rendre partie intégrante de soi-même.

Cette rigueur intellectuelle développe des habitudes et le sens de l'observation. Et ceci a pour effet de rendre la pensée plus précise et son expression plus explicite. 
Dans un autre ordre d'idées, cette racherche-action aura permis d'approfondir davantage le processus financier d'une coopérative d'épargne et de crédit. Comme administrateur d'une caisse populaire et comme professeur d'éducation économique, la dimension financière de l'entreprise passait souvent au deuxième plan. Car, ayant une formation en gestion des ressources humaines, les relations humaines occupaient la presque totalité de la place dans notre pensée administrative.

Enfin, l'enrichissement humain que les nombreux échanges et entrevues ont entraînés vont certes contribuer à remodeler les relations avec la collectivité des humains nous entourant.

CONCLUSION

En résumé, on peut affirmer que notre recherche-action est réussie. Les trois niveaux du savoir humain ont subi des transformations.

Le savoir cognitif a été enrichi de connaissances financières, de connaissance des mécanismes de résolutions de problèmes en système souple.

Le savoir-faire a été amélioré par la rigueur intellectuelle qui s'est enracinée en notre esprit.

Et enfin, le savoir-être s'est développé dans de nouvelles attitudes d'écoute des personnes avec lesquelles des décisions sont à prendre. 
CONCLUSION 


\section{CONCLUSION}

Le terme de notre étude est maintenant arrivé. Quand on jette un regard vers I'arrière, c'est un regard de grande satisfaction que nous projetons face à l'ensemble d'un dossier qu'il a fallu au moins deux ans à monter. Fruit d'une oeuvre de concertation, l'étude du Point de services fournit deux dimensions, deux raisons à notre satisfaction. Nous avons pu trouver une solution face à la problématique du Point de services. Aussi, la méthode utilisée a contribué à enrichir notre perception des choses et des personnes.

Le Point de services de Saint-Jean-Eudes, pour les administrateurs et le personnel de la Caisse, possédait une certaine valeur. On lui accordait une importance et un intérêt relatif. Cependant, on savait qu'il fallait lui conserver son existence. En faire le tour, un portrait le plus fidèle possible aura permis de découvrir qu'on minimisait sa valeur et son importance. En plus, ses faiblesses privaient la population du secteur de certains services qu'elle était en droit d'obtenir. Même que ces faiblesses pouvaient à la longue entacher sa rentabilité financière. Grâce à la méthode du diagnosticintervention, il a été possible d'imaginer, de concevoir un processus visant à permettre à la Caisse populaire d'Arvida de raffermir son marché et son rôle socio-économique à Saint-Jean-Eudes. Mais parce que la rénovation du siège social de la Caisse monopolise la majorité des énergies humaines, financières et techniques disponibles pour les améliorations, la mise en opération des mécanismes de solution des problèmes du Point de services a été retardée. 
Notre enrichissement personnel suite à l'utilisation du diagnostic-intervention, soit le deuxième volet de notre satisfaction, nous en tirerons toute la plénitude au cours d'études postérieures. Dans le chapitre sur la réflexion méthodologique, nous avons fait la constatation à l'effet que la méthode utilisée avait développé chez nous une rigueur intellectuelle dans l'analyse des situations diverses. Cette rigueur a donc provoqué des changements dans notre façon de percevoir les choses et les personnes. Cependant, la certitude totale de cet enrichissement nous sera confirmée dans le futur lorsque nous aurons à suivre une démarche relativement semblable pour trouver solution à une nouvelle situation problématique complexe. 
BIBLIOGRAPHIE 


\section{BIBLIOGRAPHIE}

1.

Cahier des objectifs, Jonquière, Caisse populaire d'Arvida, octobre 1985, 38 pages.

2.

Cahier des procès-verbaux des assemblées du C.A. de la caisse populaire Saint-Jean-Eudes, Jonquière, Archives, Caisse populaire d'Arvida 1950 - 1973.

3. Cahier des procès-verbaux des assemblées générales de la Caisse populaire Saint-Jean-Eudes, Jonquière, Archives, Caisse populaire d'Arvida, 1950 - 1973.

4.

Les assises en résumé $\left(14^{e}\right.$ congrès des dirigeants et dirigeantes de Desjardins), Lévis, C.C.P.E.D.Q., Déc. 1986, 11 pages.

5. Liquidité et capitalisation 1985, Lévis, C.C.P.E.D.Q., 1985, 37 pages.

6. Manuel SOC, Québec, Fédération des caisses populaires Desjardins de Québec, Sept. 1979.

7. Procès-verbal de l'assemblée de fondation de 1a Caisse populaire Saint-Jean-Eudes, Métabetchouan, Archives, Féd. des Caisses pop. Desjardins du Saguenay-Lac-Saint-Jean, 7 septembre 1950 .

8. Profil de marché, Lévis, C.C.P.E.D.Q., 1986, tiré à part par la Féd. des Caisses pop. Desjardins, du SaguenayLac-Saint-Jean, Pp. 123 - 133.

9. Profil du mouvement coopératif au Québec, Québec, OPDQ, 1979, Pp. $85-86$.

10. Rapports d'inspection de la Caisse populaire Saint-Jean-Eudes 1951-1973, Jonquière, Archives, Caisse populaire d'Arvida.

11. Rapport du comité de liquidité, Lévis, C.C.P.E.D.Q., Déc. 1981,117 pages.

12. Résumé, statistiques des résultats des caisses Desjardins en 1985, Lévis, C.C.P.E.D.Q., 1986, Dossier 11, 35 pages.

13. Audet, Réjean, Les coopératives financières et le développement régional, Revue Prothée, Vol. VIII,No. 3, Aut. 1980, Pp. $69-87$. 
14. Beaulieu, Rémy, Le bilan social en milieu coopératif, Chicoutimi, LEER, Automne 1983, 81 pages.

15. Beaulieu, Rémy, Charbonneau, Roland, Fabien, Louis, Perspectives socio-culturelles du développement coopératif régional, Chicoutimi, LEER, 1981, 25 pages.

16. Bernier, Guy, Entre nous, Revue Ma Caisse, Vol. 23. No.7, Nov. Déc. 1986, p.4.

17. Blais Raymond, Le bénévolat, Lévis C.C.P.E.D.Q., 1986, Dossier 10, 4 pages.

18. Blais, Raymond, Message du Président à l'assemblée générale du M.C.P.E.D. en mars 1986, Lévis, C.C.P.E.D.Q., 1986, Dossier 12, 23 pages.

19. Charbonneau, Roland, Fabien, Louis, Les valeurs et comportements coopératifs et le développement réglonal: une proposition de recherche, Chicoutimi, LEER, 1981, 14 pages.

20. Collaboration, Les Valeurs permanentes du Mouvement Desjardins, Revue Ma Caisse, Vo1. 23, No.7, Nov, - Déc. 1986, Pp. $11-14$.

21. Dallaire, Rosemarie, Trude1, Dominique, La problématique et les perspectives d'avenir dans les coopératives, Chicoutimi, LEER, Aut. 1979,38 pages.

22. Desjardins, Alphonse, Réflexions d'Alphonse Desjardins, Lévis, C.C.P.E.D.Q., 1986, 79 pages.

23. Fournier, Claire, La prise de décision dans les coopératives et le développement régional, Revue Prothée, VoI. VIII, No.3, Aut. 1980, Pp. $11-32$.

24. Gagné, Jean-Paul, $14^{e}$ congrès du Mouvement Desjardins du 28 au 30 novembre, Caisse pop: redonner la priorité aux valeurs coopératives, journal les Affaires, Samedi 22 novembre 1986 , Pp. $12-13$.

25. Gagné, Jean-Paul, 3400 dirigeants de caisses se réunissent en congrès. Le défi des caisses pop: concilier valeurs coopératives et efficacité, Journal Les Affaires, Samedi 22 novembre 1986, Pp. 10-11.

26. Giroux, Martine, Témoignage, Revue Ma caisse, Vol. 23, No.7, Nov. - Déc. 1986, pp. $15-17$. 
27. Goulet, Pierre, Cameron, Guy, Desjardins de 1900 à I'an 2000, Revue Ma Caisse, Vo1. 23, No.2, Mars - avril 1986, Pp. 5- 14.

28. Prévost, Paul, Du développement coopératif au développement régional, Revue Prothée, Vol. VIII, No.3, Aut. 1980, Pp. $11-32$.

29. Prévost, Paul, La coopération et le développement régional, Chicoutimi, LEER, 1981, 14 pages.

30. Prévost, Paul, L'organisation, un système d'activités humaines: Conception d'un modèle général, Chicoutimi, LEER, 1980, 43 pages.

31. Prévost, Paul, Le diagnostic-intervention: Une approche systémique au diagnostic organisationnel et à la recherche-action, Chicoutimi, LEER, 1983, 92 pages. 
ANNEXES 


\section{ANNEXE I}

\section{DIRIGEANTS FONDATEURS DE LA}

CAISSE POPULAIRE SAINT-JEAN-EUDES

I- Conseil d'administration:

1. M. Charles Eugène Ratté. Président

2. M. Dorillas Roy. Vice-président

3. M. Léonce Bilodeau. Secrétaire-Gérant

4. M. Roger Jean

5. M. Germain Lavoie

6. M. Jean-Louis Chayer

7. M. Pierre Roy

II- Commission de crédit:

1. M. Eugène Pedneault. Président

2. M. Henri Dechamplain

3. M. Elie Lachance

III- Commission de surveillance:

1. M. Adrien Gagnon. Président

2. M. Marcellin McNicoll

3. M. M. Joseph Bouchard

IV- Officier Honoraire

1. M. Marcel Gauthier. Prêtre curé.

Source: Archives, Fédération des Caisses populaires Desjardins du Saguenay-Lac-Saint-Jean; Métabetchouan, Procès verbal assemblée de Fondation de la Caisse Populaire Saint-Jean-Eudes, le 7 sept. 1950. 


\author{
ANNEXE II \\ La caisse populaire de Saint-Jean-Eudes \\ Etat de l'actif et du passif
}

Au 31 mai 1973

$\overline{\overline{\text { Actif }}}$

Encaisse et fonds de gestion -U.R.

$282293 \$$

Subvention à recevoir (note 1)

1623

Placement dans les organismes

du Mouvement Desjardins

3239

Actif liquide

$287255 \$$

$287155 \$$

Prêts

Sur reconnaissance de dette

98935

Moins: provision pour créances douteuses

2377

96558

Sur hypothèques

31793

Immobilisations - au coût

Ameublement et équipement

\begin{tabular}{ll}
8 & 329 \\
\hline & 994
\end{tabular}

Moins: Amortissement accumulé

3994

4335

Améliorations locatives

Moins: amortissement accumulé

2337

981

1356

Autres éléments d'actif

Intérêts gagnés non perçus

1286

Autres

652

1938

Grand total de 1'actif $\quad 423 \quad 135 \quad \$$

Signé pour le conseil d'administration 
Capital social

Epargne
Dû aux membres

Autres éléments de passif

- Exigibilités diverses

8274

- Comptes à payer et frais courus 428

$\begin{array}{ccc}\text { Total } & 8702 \$ & 8702 \\ \text { Grand total du passif } & 423135 \$\end{array}$

RAPPORT DU VERIFICATEUR

Aux membres de 1a Caisse populaire de Saint-Jean-Eudes.

Nous avons examiné l'état de l'actif et du passif de la Caisse populaire de Saint-Jean-Eudes régie par la loi des Caisses d'épargne et de crédit au 31 mai 1973, 1'état des revenus et dépenses pour 1'exercice terminé à cette date. Notre examen a comporté une revue générale des procédés comptables, ainsi que les sondages des registres comptables et autres preuves à l'appui que nous avons jugés nécessaires dans les circonstances.

A notre avis, ces états financiers présentent fidèlement la situation financière de la Caisse au 31 mai 1973, ainsi que ses résultats d'exploitation pour l'exercice terminé à cette date conformément aux principes comptables généralement appliqués de la même manière qu'au cours de $I^{\prime}$ exercice précédent.

Le service de I'inspection

Lévis, QUE.

Le 5 juin 1973

R. Gaston Bouchard

Directeur adjoint

La Fédération de Québec des Caisses populaires Desjardins 


\author{
La Caisse populaire de Saint-Jean-Eudes \\ Etat des revenus et dépenses \\ pour I'exercice terminé le 31 mai 1973
}

REVENUS:

Intérêts sur reconnaissances de dette

$8833 \$$

Intérêts sur hypothèques

3478

Intérêts sur 1'actif liquide

15756

Autres revenus

3842

$31909 \$$

DEPENSES :

Intérêts sur les dépôts

$14251 \$$

Salaires et avantages marginaux

8201

Assurance-vie sur les dépôts

1202

Assurance-vie sur les prêts

942

Contributions à l'Union régionale

1071

Papeterie, impression et dépenses de bureau

743

Assurance garantie globale et autres

578

Dons et souscriptions

31

Propagande et éducation

699

Entretien et réparations

146

Amortissement, ameublement et équipement

973

Téléphones et télégrammes

330

Frais de garde des valeurs et d'obtentions de numéraire

302

Protection

Frais juridiques et pertes sur prêts

Dépenses diverses

Coût du local

1900

Amortissement améliorations locatives

DEFICIT AVANT POSTE EXTRAORDINAIRE

1623

MOINS: SUBVENTION DU FONDS DE SECURITE (note 1)

Note 1. Subvention à recevoir du Fonds de Sécurité de la Fédération pour combler le déficit de l'exercice.

Note 2. La caisse populaire de Saint-Jean-Eudes et celle d'Arvida ont été fusionnées en date du ler juin 1973. L'actif de la Caisse populaire d'Arvida s'élève à 6608709 \$̀ à cette date. La nouvelle Caisse résultant de la fusion portera le nom de "La Caisse populaire d'Arvidal. 
ANNEXE III

La Caisse populaire d'Arvida

Etat de 1'actif et du passif au 31 mai 1973

$\overline{\text { Actif }}$

Encaisse et fonds de gestion - U.R.

$3583768 \$$

Placements

Obligations - au coût

Autres - valeur nette (note 4)

$39788 \$$

2552

Total

$42340 \$$

42340

Placements dans les organismes

du Mouvement Desjardins

28200

Actif liquide

$3654308 \$$

Prêts

Sur reconnaissances de dette

Moins: provision pour créances

douteuses

1545652

$18 \quad 318$

Total 1527334 \$

1527334

Sur hypothèque

1171745

Immobilisations - au coût

Terrain

20410

Immeuble

$198 \quad 630$

Moins: amortissement accumulé

36709

161921

Ameublement et équipement

$36 \quad 329$

Moins: amortissement accumulé

24224

12105

Autres éléments d'actif

Intérêts gagnés non perçus

41605

Impôt payé d'avance

13916

Autres

5365

Grand total de 1'actif

60886

$\begin{array}{llll}6 & 608 & 709 & \$\end{array}$

Signé par le Conseil d'administration

Président

Secrétaire 
Capital social

Epargne

Dû aux membres
$561555 \$$

$5 \quad 722 \quad 683$

$6284238 \$$

$6284238 \$$

Autres éléments du passif

- Intérêts courus non payés

- Exigibilités diverses

- Comptes à payer et frais courus

Tota1

Trop-perçus non répartis (notes 2)

Réserve générale
53548

$37 \quad 167$

5835

$96550 \$$

$6380788 \$$

$40 \quad 032$

187889

$6 \quad 608709 \$$

RAPPORT DU VERIFICATEUR

Aux membres de la Caisse populaire d'Arvida.

Nous avons examiné 1'état de 1'actif et du passif de la Caisse populaire d'Arvida régie par la loi des Caisses d'épargne et de crédit au 31 mai 1973, I'état de la réserve et des trop-perçus non repartis et l'état des revevus et dépenses pour I'exercice terminé à cette date. Notre examen a comporté une revue générale des procédés comptables, ainsi que les sondages des registres comptables et autres preuves à l'appui que nous avons jugés nécessaires dans les circonstances.

A notre avis, ces états financiers présentent fidèlement la situation financière de la Caisse au 31 mai 1973, ainsi que ses résultats d'exploitation pour l'exercice terminé à cette date conformément aux principes comptables généralement reconnus appliqués de la même manière qu'au cours de I'exercice précédent.

Le Service de 1'inspection

Lévis, QUE.

Le 8 Juin 1973

Chef de région

La Fédération de Québec des Caisses populaires Desjardins 
La Caisse populaire D'Arvida

Etat des revenus et dépenses

pour I'exercice terminé le 31 mai 1973

REVENUS :

Intérêts sur reconnaissances de dette

$151506 \$$

Intérêts sur hypothèques

79927

Intérêts sur l'actif liquide

174094

Autres revenus

$34 \quad 658$

Total

440185

DEPENSES :

Intérêts sur les dépôts

Salaires et avantages marginaux

Assurance-vie sur les dépôts

Assurance-vie sur les prêts

Contributions à l'Union régionale

Papeterie, impression et dépenses de bureau

Traitement des données (S.I.C.)

Rapport annuel et assemblée générale

Assurance garantie globale et autres

Dons et souscriptions

Propagande et éducation

Entretien et réparation

Location d'équipement pour infirmatique

Amortissement, ameublement et équipement

Téléphones et télégrammes

Frais de garde des valeurs et d'obtention

de numéraire

$218743 \$$

$\begin{array}{ll}71 & 818\end{array}$

10220

17857

14198

4948

5381

63

2700

367

8971

1385

4774

2548

747

Protection

Bien-être des employés

Frais juridiques et pertes sur prêts

810

100

7287

8128

812

Dépenses diverses

498

Coût au local:

Amortissement de I'immeuble

Dépenses de $I^{\prime}$ immeuble

$4965 \$$

7837

Moins: loyers perçus

(6 960)

Total

$5842 \$$

5842

$395197 \$$

$44988 \$$

TROP-PERÇU AVANT POSTES EXTRAORDINAIRES

5854

POSTES EXTRAORDINAIRES (note 4)

$39 \quad 134 \$$ 
La caisse populaire d'Arvida

Etat de la Réserve et des trop-preçus non répartis

pour l'exercice terminé le 31 mai 1973

\begin{tabular}{ll} 
Trop-perçus & Réserve \\
non répartis & générale \\
\hline
\end{tabular}

Solde au 31 mai 1972

$53293 \$ 159498 \$$

Impôt sur le revenu

$5 \quad 103$

Solde après impôt

$48190 \$ \quad 159498 \$$

Répartition

Virement à la réserve

$\left(\begin{array}{ll}28 & 220\end{array}\right)$

28220

Boni

(l $\left.\begin{array}{ll}19 & 072\end{array}\right)$

Solde après partage

898

187718

Trop-perçu de I'exercice

39134

Virement du droit d'entrée

171

Solde au 31 mai 1973

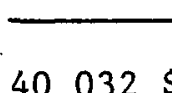

$187889 \$$

Note 1. Contrairement aux années antérieures, le trop-perçu de l'exercice a été diminué des intérêts versés sur les parts sociales pour un montant de 35667 .

Note 2. Le trop-perçu de l'exercice a été établi sans tenir compte de l'impöt sur le revenu. Le revenu imposable sur lequel l'impōt est exigible ne pouvant, en effet, être déterminé qu'après l'assemblée générale annuelle qui statue, conformément à la Loi et aux Règlements, sur la répartition des trop-perçus.

Note 3. Le coût de conversion au télétraitement s'est élevé à 14498 \$. Cependant une provision de $7000 \$$ avait été créée au 31 mai 1971 en prévision de cet événement.

Note 4. Postes extraordinaires:

Perte sur vente d'ameublement et équipement $4406 \$$

Perte sur vente d'un placement 1448

\section{$5854 \$$}

Note 5. La Caisse populaire d'Avida et celle de Saint-Jean-Eudes ont été fusionnées en date du ler juin 1973. L'actif de la Caisse populaire de Saint-Jean-Eudes d'élève à 423135 \$ à cette date. La nouvelle Caisse résultant de la fusion portera le nom de "La Caisse populaire d'Arvida". 


\author{
ANNEXE IV \\ La caisse populaire d'Arvida \\ La caisse populaire Saint-Jean-Eudes \\ Bilan consolide au 31 mai 1973
}

$\overline{\text { Actif }}$ :

Encaisse et fonds de gestion - U.R.

$3866061 \$$

Subvention à recevoir

1623

Placements :

Obligations - au coût

$39788 \$$

Autres - valeur nette

2552

42340

Placements dans les organismes du Mouvement Desjardins

Actif liquide

3941463

Prêts

Sur reconnaissances de dette

Moins: provision pour créances douteuses

$1644587 \$$

20695

1623892

Sur hypothèques

1203538

Immobilisations - au coût

Terrain

20410

Immeuble

Moins: amortissement accumulé

$198 \quad 630$

36709

161921

Ameublement et équipement

$44658 \$$

moins: amortissement accumulé

$28 \quad 218$

16440

Amélioration locative

$2337 \$$

$981 \$$

1356

Autres éléments de $I^{\prime}$ actif

Intérêts gagnés non perçus

Impôt payé d'avance

$42891 \$$

13916

Autres

$\begin{array}{lll}6 & 017\end{array}$

$62 \quad 824$ 
Passif:

Capital social

Epargne

Dû aux membres

Autres éléments du passif

Exigibilités diverses

Comptes à payer et frais courus

Total

Trop-perçus non répartis

Réserve générale
$569096 \$$

$6 \quad 129 \quad 575$

$6698671 \$$

$53548 \$$

45441

6263

$105252 \$$

105252

$40 \quad 032$

$187 \quad 889$

$7031844 \$$ 


\section{ANNEXE $V$}

Jonquière, 30 juillet 1985

A tous les sociétaires, Comptoir de la Caisse populaire d'Arvida Saint Jean-Eudes.

Madame, Monsfeur,

Je suis étudiant en maitrise de gestion des petites et moyennes organisations à l'Université du Québec à Chicoutimi.

A ma demande, les airigeants de la Caisse ont bien voulu que je fasse porter ma recherche finale sur l'importance du comptoir de la Caisse populaire d'Arvida à Saint Jean-Eudes. Pour réaliser cette étude sur la valeur du comptoir, j'aurais besoln de renseignements au sujet de votre appartenance à ce comptoir de la Caisse. Vous serait-il possible de répondre au questionnaire ci-joint et me le retourner le plus tôt fossible en vous servant de l'enveloppe pré-affranchie à cet effet?

Pour 12 réussite de mor. projet, 11 serait très important que chacun d'entre vous réponde au questionnaire et me le retourne dans les plus brefs délais.

Et dans le but de m'appuyer dans cette démarche, le directeur de la Calsse, M. Marc Laflame, foint sa signature à la mienne.

Je vous remercie à l'avance de votre collaboration empressée et vous prie d'accepter mes salutations les plus chaleureuses.

Bernard Flamand, étudiant.

Marc Laflamme, directeur. 


\section{QUESTIONNAIRE-SONDAGE}

Soumis aux sociétaires

Comptoir Saint-Jean-Eudes

Directive: Pour répondre au questionnaire, vous faites un $X$ dans l'espace requis ou vous remplissez la ligne.

1- Votre sexe:

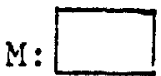

F :

2- A quel groupe d'âqe eppartenez-vous?

$$
\begin{aligned}
& 0-15 \text { ans } \\
& 16-25 \text { ans } \\
& 26-35 \text { ans }
\end{aligned}
$$

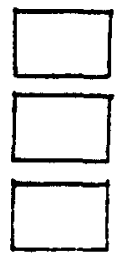

$46-55$ ans

$56-65$ ans

65 ans et pius

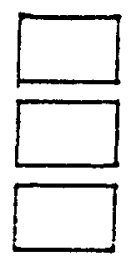

3- Que1 est votre profession?

4- A quei endroit travaillez-vous?

5- Depuis combien de ternps êtes-vous membre du Comptoir Saint-Jean-Eudes de la Caisse popilaire G'Arvida?

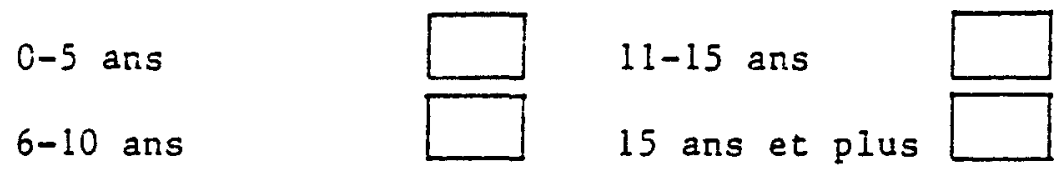

6- Combien de fois far mcis allez-vous au comptoir de la caisse à Saint-Jean-Eudes?
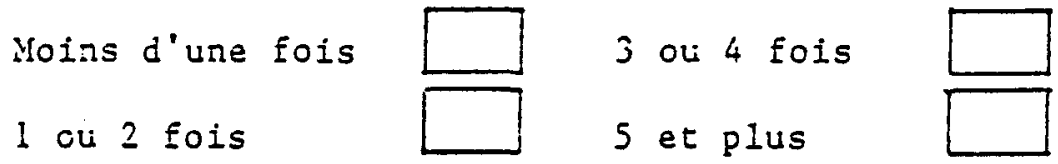

7- Parfois, faites-vous affaire directement à la Caisse populaire sur le Boul. Mellon?

Oui
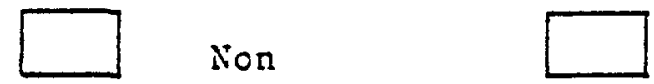
8- Si oul à la question 7, comblen de fols par mois y allez-vous?

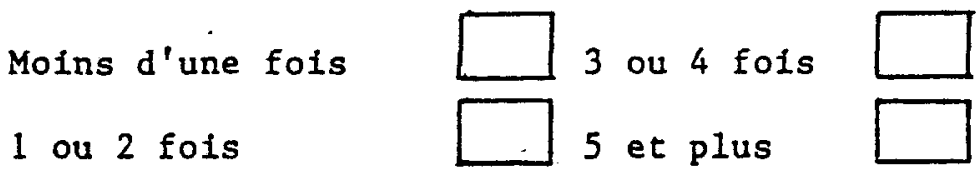

9- Votre compte d'épargne est-11 conjolnt avec quelqu'un?

Oui
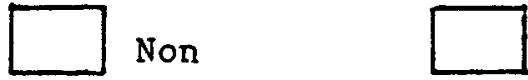

10- Si oui à la question 9, précisez.

Epoux (se)

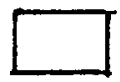

Associé

A 2 signatures

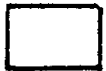

11- Si oui à la question 9, combien de fois paz zois votre parteraire utilise-t-il le comptoir Saint-Jean-Eudes?

Moins d'une fois

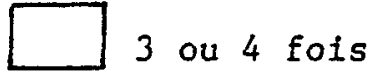

1 ou 2 fois
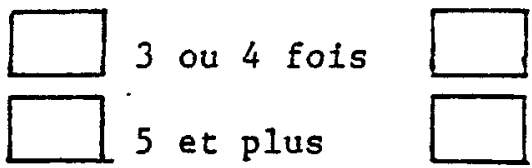

12- Si oui à la question 9, combien de fois par mois votre partenaire de compte fait-il affaire directement à la Caisse, Boul. Mellon?

Moins d'une fois

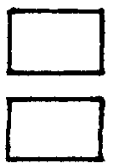

3 ou 4 fois

1 ou 2 fois

5 et plus

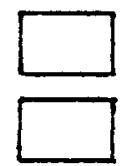

13- Avez-vous une carte de guichet automatique?

Oui

Non

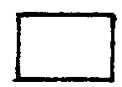

14- Si oui à la question 13, combien de fois par mols 1'utilisez-vous?

à Arvida?

ailleurs?

15- Faites-vous affaire avec une autre institution bancaire que la Caisse populaire?

Oui

Non

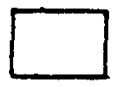

16- Si oui à la question 15, où est située cette autre institution?
A Arvida
A Jonquière

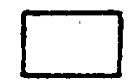

A Chicoutimi

A Kénogami

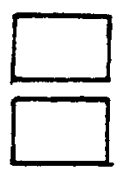


17- Si oui à la question 15, environ combien de fois par mois utilisez-vous cette autre institution bancaire?

Moins d'une fois

1 ou 2 fois

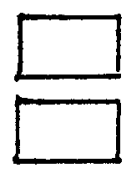

3 ou 4 fois

5 et plus

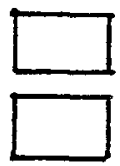

18- Si oul à la question 15, vos affaires avec l'autre institution bancafre sont-elles plus importantes en dollars que celles de la Caisse?

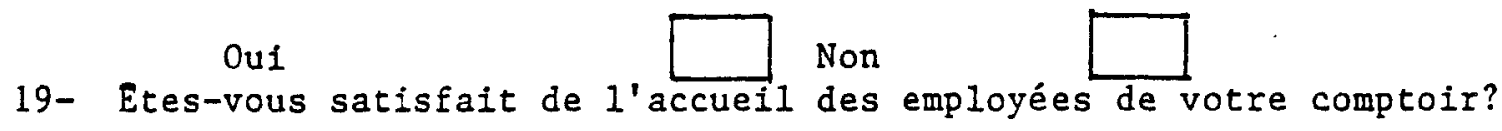
Oui
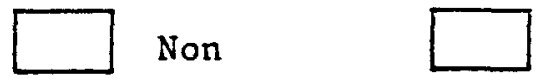

20- Etes-vous satisfaits des services offerts au comptoir Saint-Jean-Eudes?

Oui Non

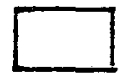

21- L'accès routier et $I^{\prime}$ accès au stationnement sont-1ls adéquats à votre comptoir?

Oui

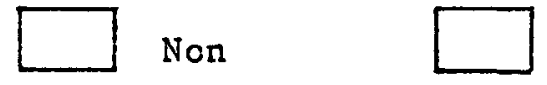

22- Sauf l'amélicration du stationnement, souhaitez-vous d'autres améliorations à votre comptoir?

Oui

Nor

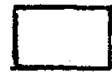

23- Si oui à la question 22, précisez 1'amélioration que vous désirez le plus à votre comptoir.

24- Si on vous disait que la Caisse ferme son comptoir à Saint-Jean-Eudes parce qu'il est non rentable ou pas assez rentable, que ferlez-vous?

Je changerais de banque

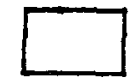

Je ferais des pressions pour le garder ouvert

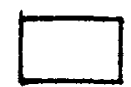

Merci de votre collaboration

Bernard Flamand 


\section{ANALYSE DU SONDAGE}

Le sondage effectué auprès des sociétaires du Point de services I'a été à la fin juillet et début août 1985. Nous avons donc couvert les deux périodes d'achalandage marqué; soit un jeudi et un vendredi comme jours de paye, soit un mardi en début du mois. Les questionnaires ont été distribués, pour la très grande majorité, de main à main par i'auteur du mémoire. Quelques'uns avaient été placés près des guichets de caisse pendant la période séparant nos deux présences au Point de services. En tout, 300 questionnaires ont été cistribués; et un peu plus des $2 / 3$ des perscnnes solicitées (soient 214) ont retourné le questionnaire. Il faut dire que beaucoup de personne ont répondu sur place pendant leur attente en file.

Voici, ce que la compilation de ce sondage a révélé. Nous faisons remarquer en premier lieu que la numérotation utilisée ici ne correspond pas nécessairement à chacun des numéros du questionnaire.

1. Sexe des répondants.

86 répondants étaient hommes $=40,18 \%$

128 répondants étaient femres $=59,82 \%$

2. Age des répondants

\begin{tabular}{ccc} 
Nombre & \multicolumn{1}{c}{ Age } & $\%$ \\
42 & $16-25$ ans & 19,63 \\
62 & $26-35$ & 28,97 \\
38 & $36-45$ & 17,76 \\
26 & $46-55$ & 12,15 \\
24 & $56-65$ & 11,21 \\
22 & 65 et plus & 10,28
\end{tabular}


$\mathrm{Si}$ on fait un calcul rapide, on constate que $58,88 \%$ des répondants sont âgés de 36 à 65 ans. On découvre aussi que $66,36 \%$ des répondants ont entre 26 et 55 ans. Quand, en plus on considère que le groupe de 16 à 25 ans représente $28,97 \%$ des répondants, la plus forte proportion, on est facilement porté à déduire que les sociétaires de Saint-Jean-Eudes sont relativement jeunes.

3. Les répondants qui étaient des femmes demeurant au foyer représentaient $28,97 \%$ de tous les répondants, soient 62 femmes.

4. Lieu de travail.

Pour $42,99 \%$ des sociétaires répondants, le lieu de travail était Alcan ou Saint-Jean-Eudes. En ce qui regarde les autres endroits de travail, la répartition est la suivante: $31,78 \%$ travaillent à Jonquière, ailleurs qu'à I'Alcan. $18,67 \%$ à Chicoutimi, $6,54 \%$ sont chômeurs.

5. Depuis quand est-on membre de la Caisse à Saint-Jean-Eudes?

\begin{tabular}{rcc} 
Nombre & \multicolumn{2}{c}{ Temps } \\
8 & -5 ans & 18,69 \\
38 & $6-10$ ans & 17,76 \\
32 & $11-15$ ans & 14,95 \\
104 & 16 ans et plus & 48,60
\end{tabular}

6. Fréquence d'utilisation du Point de services

$\begin{array}{rrr}\text { Nombre } & \text { Fréquence/mois } & \% \\ 8 & -1 \text { fois } & 3,74 \\ 24 & 1-2 \text { fois } & 11,21 \\ 82 & 3-4 \text { fois } & 38,32 \\ 100 & 16 \text { ans et plus } & 46,73\end{array}$


12. Accueil et service.

La très grande majorité des répondants (près du 100\%) se déclare satisfait de l'accueil et de la qualité des services offerts.

13. Facilité d'accès.

Environ $73 \%$ des répondants n'aiment pas $I^{\prime}$ accès routier et

le stationnement.

14. Fermeture définitive.

Si on décidait de fermer le Point de serives, $84,11 \%$ des répondants ont déclaré qu'ils feraient des pressions pour garder le Point de services cuvert.

15. Liste des principales améliorations souhaitées par ordre prioritaires.

1) Avoir un agent pour négocier les placements et surto

des prêts.

2) Améliorer le stationnement.

3) Avoir deux employées en service continu aux ' pointe, les jeudi et vendredi de paye et les débuts de mois.

4) Ouvrir les jeudis et vendredis soir jusqu'à 1 . au lieu de 18 heures; où encore revenir un peu ̀̀ l'ancienne formulc. c'est-à-dire ouvrir de 19 heures à 20 heures ces soirs-1à. 